Geomorphologie-relief Processus Environnement

July 2015, Volume 21 Issue 3 Pages 267-292

http://dx.doi.org/10.4000/geomorphologie.11104

http://archimer.ifremer.fr/doc/00310/42171/

(c) Groupe français de géomorphologie

\title{
Bilan des tempêtes de l'hiver 2013-2014 sur la dynamique de recul du trait de côte en Bretagne
}

\section{Review of winter storms 2013-2014 on shoreline retreat dynamic on Brittany coast}

\author{
Blaise Emmanuel ${ }^{1} \stackrel{*}{-}$, Suanez Serge ${ }^{1}$, Stephan Pierre ${ }^{1}$, Fichaut Bernard ${ }^{1}$, David Laurence ${ }^{1}$, \\ Cuq Veronique ${ }^{1}$, Autret Ronan ${ }^{1}$, Houron Julien ${ }^{2}$, Rouan Mathias ${ }^{1}$, Floc'h France ${ }^{3}$, Ardhuin Fabrice ${ }^{4}$, \\ Cancouet Romain ${ }^{1}$, Davidson Robert ${ }^{5}$, Costa Stephane ${ }^{5}$, Delacourt Christophe ${ }^{3}$ \\ 1 Inst Univ Europeen Mer, CNRS, LETG Brest Geomer, UMR 6554, F-29280 Plouzane, France.
2 Reserve Nat Reg Sillon Talbert, Maison Sillon, F-22610 Pleubian, France.
${ }_{3}^{3}$ Inst Univ Europeen Mer, CNRS, LDO, UMR 6558, F-29280 Plouzane, France.
${ }^{4}$ Inst Univ Europeen Mer, CNRS Ifremer IRD, LPO, UMR 6523, F-29280 Plouzane, France.
${ }^{5}$ Univ Caen Basse Normandie, CNRS, LETG Caen Geophen, UMR 6554, F-14032 Caen, France.
}

Corresponding author email address : emmanuel.blaise@univ-brest.fr; serge.suanez@univbrest.fr;;;ierre.stephan@univ-brest.fr; bernard.fichaut@univ-brest.fr;;laurence.david@univ-brest.fr; veronique.cuq@univ-brest.fr;;ronan.autret@univ-brest.fr; maison-littoralpleubian@orange.fr;;mathias.rouan@univ-brest.fr; france.floch@univbrest.fr;;Fabrice.Ardhuin@ifremer.fr; romain.cancouet@univ-brest.fr;;robert.davidson@unicaen.fr; stephane.costa@unicaen.fr;;christophe.delacourt@univ-brest.fr

\section{Résumé}

Entre les mois de décembre 2013 et mars 2014, une douzaine de tempêtes ont touché la pointe bretonne avec une fréquence exceptionnelle. C'est au mois de février que ces évènements ont été les plus fréquents et particulièrement virulents. Les hauteurs significatives des vagues mesurées au large du Finistère ont atteint respectivement $12,3 \mathrm{~m}$ et 12,4 $\mathrm{m}$ lors des tempêtes Petra et Ulla du 5 et du 14 février. L'analyse des conditions hydrodynamiques montre toutefois que seuls trois épisodes ont été particulièrement morphogènes car ils ont été combinés à des fortes marées de vive-eau ; il s'agit des évènements couvrant les périodes du 1 au 4 janvier, du 1 au 3 février, et du 2-3 mars 2014 durant lesquels les niveaux de marée observée ont été supérieurs au niveau des plus hautes marées astronomiques (PHMA). Les surcotes maximum (entre $0,80 \mathrm{~m}$ et $0,97 \mathrm{~m}$ ) ont été atteintes lors de la tempête Ulla du 14-15 février. II faut remonter à l'hiver 1989-1990 pour connaître une situation hivernale similaire, même si les tempêtes du mois de février 1990 avaient été encore plus nombreuses et plus violentes. Des mesures topo-morphologiques à haute fréquence réalisées sur une dizaine de cordons littoraux répartis sur le pourtour de la péninsule bretonne permettent d'évaluer les effets de ces évènements sur le recul du trait de côte. Elles montrent que durant la première phase (décembre- 
janvier), marquée notamment par l'épisode morphogène du 1 au 4 janvier 2014, l'érosion du rivage a été limitée, à l'exception du sud Bretagne. Cela s'explique par l'orientation plutôt sud-ouest de la houle incidente. De façon générale, le recul a atteint en moyenne $-2,7 \mathrm{~m}$ (le minimum moyenné sur l'ensemble des sites : $-0,6 \mathrm{~m}$; le maximum moyenné sur l'ensemble des sites : $-6,20 \mathrm{~m}$ ). Durant la seconde phase de mi-janvier à mi-février, caractérisée par la tempête du 1-2 février qui a été la plus morphogène de l'hiver, le recul moyen a atteint $-4,2 \mathrm{~m}$ (le minimum moyenné sur l'ensemble des sites : $-1,5 \mathrm{~m}$; le maximum moyenné sur l'ensemble des sites : $-9,5 \mathrm{~m}$ ). C'est essentiellement la côte nord et ouest de la Bretagne qui a enregistré les reculs les plus importants car la houle était alors orientée ONO. Durant la troisième et dernière phase qui s'étend de mi-février à mi-mars, marquée par l'épisode morphogène du 2-3 mars, le recul du trait de côte à été très faible. II a atteint $-1 \mathrm{~m}$ en moyenne (le minimum moyenné sur l'ensemble des sites : $-0,6 \mathrm{~m}$; le maximum moyenné sur l'ensemble des sites : $1,9 \mathrm{~m}$ ). À l'échelle de l'hiver 2013-14, l'érosion du rivage pour l'ensemble des sites observés a atteint 6,3 $\mathrm{m}$ en moyenne (minimum : -0,2 $\mathrm{m}$; maximum : $-30,1 \mathrm{~m}$ ). Lorsque l'on observe plus en détail ces chiffres, il apparaît que ce sont les cordons dunaires qui ont le plus reculé, viennent ensuite les formes fuyantes à pointe libre de type flèches sableuses ou les cordons de galets; les reculs les plus faibles concernent les plages adossées ou les falaises entaillées dans des matériaux cohérents comme les plages pléistocènes ou les falaises de head. La réponse des plages aux trois épisodes morphogènes a été différente. Celui du début du mois de février reste le plus érosif et s'explique en grande partie par une plus forte sensibilité des cordons préalablement fragilisés par l'épisode du début du mois de janvier. À l'inverse, la tempête du mois de mars n'a pratiquement eu aucun impact. Ces éléments montrent qu'il n'y a aucun effet cumulatif attesté de l'effet des tempêtes. Sur une longue période marquée par une série d'évènements tempétueux, au-delà d'un certain seuil dans le processus de recul du rivage, l'action érosive des épisodes morphogènes n'agit plus de façon significative, quelle que soit leur intensité.

Mots-clés : tempête, érosion, trait de côte, marée de vive-eau, Bretagne, surcote

\begin{abstract}
:
Between December 2013 and March 2014, a cluster of about 12 storm events hit the coast of Brittany with an exceptional frequency. It was in February that these storm events were the most frequent and particularly virulent. The significant wave heights measured off Finistere reached respectively $12.3 \mathrm{~m}$ and $12.4 \mathrm{~m}$ during Petra and Ulla storms on February 5 and 14. However, analysis of hydrodynamic conditions shows that only three episodes promoted extreme morphogenetic conditions because they were combined with high spring tide level. The first one occurred on January 1 to 4 , it was followed by events from February 1 to 3, and March 2-3. During these three extreme events observed tide levels were above highest astronomical tide level (HAT). Maximum surge level $(0.97 \mathrm{~m})$ was reached during Ulla storm of February 14-15. For comparison, we must go back in the winter of 1989-90 to find such extreme storm frequency. High frequency topomorphological measurements were achieved on more than ten coastal zones distributed around Brittany peninsula to assess the effects of these storms on shoreline erosion. They show that during the first phase (December-January), meeting it's climax from 1,1 to 4(th) of January 2014, shoreline erosion has been limited, with the exception of southern Brittany. This is due to the SW orientation of waves. For all monitoring sites, it has averaged $-2.7 \mathrm{~m}$, the averaged minimum equal to $0.6 \mathrm{~m}$, and the averaged maximum at $-6.20 \mathrm{~m}$. During the second phase from mid-January to mid-February, reaching it's climax on 1-2 of February storm corresponding to the most morphogenetic event of the winter, the average of shoreline retreat reached $-4.2 \mathrm{~m}$, the averaged minimum reached approximately $-1.5 \mathrm{~m}$, the averaged maximum $-95 \mathrm{in}$. It is essentially the Northern and Western coast of Brittany that experienced largest shoreline retreat due to W-NW storm wave orientation. During the third and last phase, running from mid-February to mid-March, and characterized by the March 2-3 extreme morphogenetic event, shoreline retreat was very low. It reaches $-1 \mathrm{~m}$ on average, for an averaged minimum of $-0.6 \mathrm{~m}$ and an averaged minimum of $-1.9 \mathrm{~m}$. Considering the whole winter 2013-14 period, shoreline erosion for all monitoring sites reached $-6.3 \mathrm{~m}$ on average, with a minimum of about -0.2 in and a maximum of $-30.1 \mathrm{~m}$. Depending on the type of environment, it
\end{abstract}


appears that the dunes have retreated the most, followed by gravel or sandy barriers; the lowest erosion rates concern beaches backed by low cliffs cut in highly consistent materials such as periglacial deposits (head). Considering the three morphogenous episodes, the morphological response in terms of shoreline retreat of beaches and barriers was different. Storm occurring at the beginning of February induced the largest erosive rates partly explained by the large morphological sensitivity of beaches and barriers which were weakened by the previous storm events in the beginning of January. Conversely, the storm of March induced very few impacts. These elements show that there is no cumulative of storm effect attested. Over a long period marked by a cluster of storms, beyond a certain threshold in the shoreline retreat process, the erosive action of morphogenesis events is no longer significant, regardless of their intensity.

Keywords : storm, high spring tide, surge, erosion, shoreline, Brittany 


\section{Plan}

1 . Introduction

2. Les tempêtes de l'hiver 2013-2014

3. Les sites d'étude

4. Méthode et données

4.1. Analyse des conditions météomarines et hydrodynamiques

4.2. Suivis morphologiques

$\underline{5 . \text { Résultats }}$

5.1. Bilan global sur le recul du rivage

5.2. Erosion des dunes

5.3. Recul des cordons de galets

5.4. Impact sur les cordons littoraux anthropisés et/ou protégés

6. Discussion

7. Conclusion

\section{Notes de la rédaction}

Article soumis le 16 décembre 2014, reçu sous sa forme révisée le le 13 mars 2015, définitivement accepté le 12 avril 2015.

Ce travail a bénéficié d'une aide de l'Etat gérée par l'Agence Nationale de la Recherche dans le cadre du LabexMer au titre du programme «Investissements d'avenir » portant la référence ANR-10-LABX-19-01, et de l'ANR COCORISCO au titre du programme : Changements Environnementaux Planétaires \& Sociétés (CEP\&S) 2010, portant la référence ANR-10CEPL-0001. Il a aussi bénéficié d'un soutien financier de l'INSU dans le cadre de l'observatoire du domaine marin de l'IUEM (Observatoire du Domaine Côtier), et du Service National d'Observation - SNO -DYNALIT, et de AllEnvi (l'alliance nationale de recherche pour l'environnement qui regroupe le Ministère de l'Enseignement Supérieur et Recherche et la Direction Générale Recherche et Innovation) dans le cadre du SOERE trait de côte, aménagements littoraux (Système d'Observation et d'Expérimentation à long terme pour la Recherche en Environnement). Les observations des marégraphes de Roscoff, du Conquet, de Concarneau et de Brest, sont la propriété du SHOM, de la CCI Morlaix et sont mises à disposition sur le site des Réseaux de référence des observations marégraphiques (http://refmar.shom.fr).

\section{Introduction}

1L'hiver 2013-2014 a été caractérisé par une succession de tempêtes qui ont touché la péninsule bretonne entre le mois de décembre 2013 et le mois de mars 2014. Ces épisodes ont été particulièrement fréquents, notamment durant le mois de février, et ont provoqué de graves dommages en matière d'érosion et de submersion marine. De nombreuses communes littorales des départements bretons, notamment du Finistère et des Côtes d'Armor, ont subi de nombreux dégâts tant au niveau des infrastructures anthropiques que des milieux naturels. L'impact des tempêtes sur l'évolution morphosédimentaire des plages est déterminant car ces évènements extrêmes commandent les changements à très court terme du trait de côte (Dolan et Hayden, 1981 ; Balsillie, 1986 ; Morton et al., 1995 ; Fenster et al., 2001 ; Honeycutt et al., 2001 ; Forbes et al., 2004 ; Houser et al., 2008 ; Sénéchal et al., 2015). Ils interviennent également dans les échanges sédimentaires complexes entre les zones infra, médio et supra- 
littorales qui contrôlent notamment les variations du profil transversal des plages (Birkemeier et al., 1999 ; Lee et al., 1998 ; Aagaard et al., 2005 ; Stone et al., 2004 ; Ferreira, 2006 ; Costa et al., 2008 ; Anthony, 2013). Toutefois, la réponse morphosédimentaire des plages à ces évènements tempétueux est aussi dépendante de leur fréquence par rapport aux périodes de régénération qui les séparent; l'érosion est généralement accentuée lorsque cette fréquence excède la durée de résilience post-tempête du milieu après chaque épisode (Morton et al., 1995 ; Anthony, 2013). Cela pose la question de l'impact morphosédimentaire d'une succession de tempêtes sur un pas de temps très court. Plusieurs études ont montré que dans certains cas, l'érosion combinée de plusieurs épisodes tempétueux est plus importante que la somme du recul moyen généré par une seule tempête (Morton, 2002 ; Lee et al., 1998). De même, une succession très rapide d'évènements tempétueux de faible intensité peut produire autant de dommages qu'une seule tempête très virulente (Birkemeier et al., 1999; Cox et Pirrello, 2001 ; Ferreira, 2005). Il n'est donc pas facile de déterminer des seuils morphogènes de tempête pourtant utiles lorsqu'il s'agit d'évaluer le risque d'érosion dans le but de protéger et/ou d'aménager des secteurs côtiers (Gibeaut et al., 2002; Zielinski, 2002; Mendoza et Jiménez, 2006 ; Ciavola et al., 2007 ; Stockdon et al., 2007 ; Gervais et al., 2012 ; Bosom et Jimenez, 2010 ; Rangel-Buitrago et Anfuso, 2011 ; Almeida et al., 2012 ; Jimenez et al., 2012). La hiérarchisation de tempêtes successives reposant sur des critères hydrodynamiques reste difficile à faire car le contexte marégraphique (situation de vive-eau ou de morte-eau), les caractéristiques de la houle au large, et les conditions morphodynamiques dans la zone de déferlement sont autant de paramètres qui rendent l'analyse des processus extrêmement complexe, et ne permettent pas d'anticiper la réponse morphosédimentaire de la plage à chaque épisode morphogène (Yates et al., 2009 ; Anthony, 2013 ; Coco et al., 2014 ; Castelle et al., 2014). De même, la morphologie pré-tempête est un facteur déterminant car elle contrôle la réponse morphosédimentaire des cordons littoraux en intervenant sur les forçages hydrodynamiques, en particulier l'action des niveaux d'eau extrêmes à la côte qui devient plus importante au cours des dernières tempêtes lorsque le profil de plage s'est abaissé (Caspar et al., 2010 ; Vousdoukas et al., 2012 ; Letortu et al., 2012 ; Splinter et al., 2014). Si des seuils morphogéniques peuvent être définis en fonction des caractéristiques hydrodynamiques et météomarines propres à chaque épisode tempétueux, il est pour autant impossible d'établir une classification pertinente de ces derniers en fonction de la réponse morphosédimentaire des plages (Haerens et al., 2012). Qui plus est, si l'intensité des tempêtes peut répondre à un fonctionnement saisonnier, leur impact morphosédimentaire est aussi fonction du caractère morphodynamique des plages (Rangel-Buitrago et Anfuso, 2011; Aagaard et al., 2012 ; Armaroli et al., 2013 ; Dubois et al., 2014 ; Pian et al., 2014). Ainsi, les plages de type intermédiaire montrent le plus souvent des changements de profil plus importants que celles qui observent un caractère dissipant.

2L'objectif de cet article est de faire le bilan morphosédimentaire des tempêtes de l'hiver 2013-2014, notamment en matière de dynamique de recul du trait de côte. Dans un premier temps, une analyse des conditions hydrodynamiques et météomarines permet de caractériser cette série d'évènements tempétueux et de discriminer les épisodes les plus morphogènes ayant contribué à l'érosion du littoral. Dans un second temps, les mesures topomorphologiques réalisées sur une dizaine de sites répartis sur le pourtour du littoral breton permettent d'évaluer l'impact de ces tempêtes sur la dynamique de recul du trait de côte. L'objectif est d'analyser la variabilité de la réponse des cordons littoraux en fonction de leur caractéristiques géomorphologiques et dynamiques propres. Enfin, nous discutons cette série de tempêtes tant du point de vue de la fréquence et de l'intensité, que de ses effets sur la dynamique érosive, en la replaçant au sein d'une chronologie d'évènements tempétueux remontant à plusieurs décennies. 


\section{Les tempêtes de l'hiver 2013-2014}

3Un inventaire des tempêtes de l'hiver 2013-2014 a été réalisé pratiquement en temps réel à partir des observations et des modélisations numériques de houle produites dans le cadre du projet PREVIMER coordonné par l'Ifremer et le SHOM (http://www.previmer.org). Cette plateforme numérique fournit en routine des observations et des prévisions sur l'état de l'environnement marin dans la zone côtière (courants, vagues, températures, salinité, etc.), le long des grandes façades régionales de la Manche, de l'Atlantique et de la Méditerranée pour la France métropolitaine, ainsi que certains départements et territoires d'outre-mer. Les modélisations atteignent des résolutions de $300 \mathrm{~m}$ à la côte; elles sont ajustées à grande échelle sur des mesures satellites historiques (Rascle et Ardhuin, 2013 ; Roland et Ardhuin, 2014), et validées avec des mesures in situ. D'un point de vue temporel, PREVIMER couvre à la fois des analyses rétrospectives, des prévisions à court terme ( $96 \mathrm{~h}$ à $120 \mathrm{~h}$ pour les vagues, utilisant les prévisions de vent du Centre Européen de Prévisions ECMWF), et une prévision harmonique des courants et des niveaux d'eau associés aux marées. Notre inventaire de tempêtes a été réalisé à partir des statistiques de houle moyennes et maximales calculées sur différentes fenêtres d'environ $60 \mathrm{~km}$ de largeur, centrées sur les secteurs faisant l'objet de cette étude (fig. 1).

Fig. 1 - Localisation des fenêtres de calcul des paramètres statistiques de houle issues du modèle PREVIMER.

Fig. 1 - Location of windows for wave statistic parameters calculation provided by PREVIMER modeling.

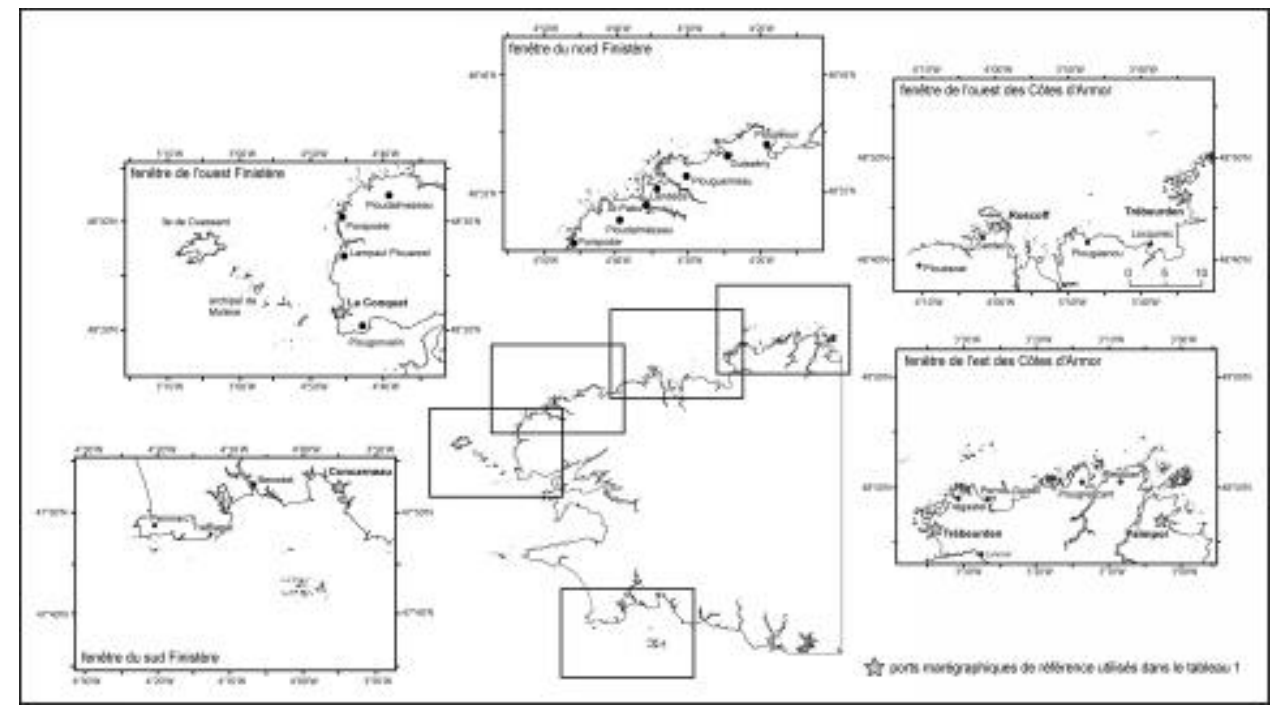

$\underline{\text { Agrandir }} \underline{\text { Original (jpeg, 772k) }}$

Source : http://www.previmer.org

Source: $\underline{\text { http://www.previmer.org }}$

4Les valeurs qui ont été retenues correspondent aux hauteurs de houle acquises au moment de la pleine-mer dont l'heure est fournie par les enregistrements marégraphiques réalisés aux ports de Paimpol, de Trébeurden, de Roscoff, du Conquet et de Concarneau, respectivement pour les fenêtres des Côtes d'Armor est et ouest, du nord, de l'ouest et du sud Finistère. Cette information, bien que quantitative, n'est pas vraiment exploitable dès lors que l'on s'intéresse 
à un secteur de plage bien précis car elle est calculée sur un très large périmètre et non en un point donné. Toutefois, elle a permis de lister et de hiérarchiser une douzaine d'épisodes tempétueux survenus entre la fin du mois de décembre 2013 et le début du mois de mars 2014 (tab. 1).

Tab. 1 - Inventaire des tempêtes de l'hiver 2013-2014.

Tab. 1 - Inventory of winter 2013-14 storms. 


\begin{tabular}{|c|c|c|c|c|c|c|}
\hline $\begin{array}{l}\text { Dates et } \\
\text { noms des } \\
\text { tempétes }\end{array}$ & & $\begin{array}{c}\text { Cotes } \\
\text { d'Armor est } \\
\text { Paimpol }\end{array}$ & $\begin{array}{l}\text { Côtes } \\
\text { d'Armor } \\
\text { ouest } \\
\text { Treberden }\end{array}$ & $\begin{array}{l}\text { Nord } \\
\text { Finistère } \\
\text { Roscoff }\end{array}$ & $\begin{array}{c}\text { Ouest } \\
\text { Finistère } \\
\text { Le Conquet }\end{array}$ & $\begin{array}{l}\text { Sud Finistère } \\
\text { Concarneau }\end{array}$ \\
\hline date & $\begin{array}{l}\text { coeff. } \\
\text { de } \\
\text { marée }\end{array}$ & $\begin{array}{c}\text { hauteurs de } \\
\text { houle a } \\
\text { pleine mer } \\
\text { (m) }\end{array}$ & $\begin{array}{l}\text { hauteurs de } \\
\text { houle à } \\
\text { pleine mer } \\
\text { (m) }\end{array}$ & $\begin{array}{l}\text { hauteurs de } \\
\text { houle à } \\
\text { pleine mer } \\
\text { (m) }\end{array}$ & $\begin{array}{l}\text { hauteurs de } \\
\text { houle a } \\
\text { pleine mer } \\
\text { (m) }\end{array}$ & $\begin{array}{l}\text { hauteurs de } \\
\text { houle a } \\
\text { pleine mer } \\
\text { (m) }\end{array}$ \\
\hline $23 / 12 / 2013$ & $23 / 12$ & $23 / 12-09 \mathrm{~h}$ & $23 / 12-08 \mathrm{~h}$ & $23 / 12-08 \mathrm{~h}$ & $23 / 12-07 \mathrm{~h}$ & $23 / 12-08 \mathrm{~h}$ \\
\hline $24 / 12 / 2013$ & $61-58$ & moy : 2,68 & moy : 3,37 & moy $: 4.14$ & moy $: 4.12$ & moy $: 3.63$ \\
\hline $25 / 12 / 2013$ & $24 / 12$ & $\max : 4,19$ & $\max : 4,70$ & $\max : 5.19$ & $\max : 5.84$ & $\max : 4.99$ \\
\hline tempête Dirk & $54-51$ & $23 / 12-21 \mathrm{~h}$ & $23 / 12-21 \mathrm{~h}$ & $23 / 12-21 \mathrm{~h}$ & $23 / 12-20 \mathrm{~h}$ & $23 / 12-20 h$ \\
\hline & $25 / 12$ : & moy : 3,51 & moy : 4,23 & moy : 5.31 & moy : 5.88 & moy : 5.96 \\
\hline & & $\max : 5,80$ & $\max : 6,41$ & $\max : 7.51$ & $\max : \mathbf{8 . 3 0}$ & $\max : 7.70$ \\
\hline & & $24 / 12-09 \mathrm{~h}$ & 24/12-09h & $24 / 12-09 \mathrm{~h}$ & $24 / 12-08 \mathrm{~h}$ & $24 / 12-09 h$ \\
\hline & & moy : 3.21 & moy : 3,98 & moy $: 4.97$ & moy : 5.19 & moy : 5.18 \\
\hline & & $\max : 5,08$ & $\max : 5,71$ & $\max : 6.50$ & $\max : 7.36$ & $\max : 6.87$ \\
\hline & & $24 / 12-22 h$ & $24 / 12-22 \mathrm{~h}$ & $24 / 12-22 h$ & $24 / 12-20 h$ & $24 / 12-21 h$ \\
\hline & & moy : 3,24 & moy : 4.07 & moy : 5.13 & moy $=5.00$ & moy $: 4.36$ \\
\hline & & $\max : 5,05$ & $\max : 5,64$ & $\max : 6.44$ & $\max =7.18$ & $\max : 6.03$ \\
\hline & & $25 / 12-10 \mathrm{~h}$ & $25 / 12-10 \mathrm{~h}$ & $25 / 12-11 \mathrm{~h}$ & $25 / 12-09 h$ & $25 / 12-10 \mathrm{~h}$ \\
\hline & & moy : 2,98 & moy : 3,67 & moy: 4.31 & moy: 4.46 & moy: 3.74 \\
\hline & & $\max : 4,58$ & $\max : 4,93$ & $\max : 5.25$ & $\max : 6.26$ & $\max : 5.38$ \\
\hline $27 / 12 / 2013$ & $27 / 12$ & $27 / 12-00 \mathrm{~h}$ & $27 / 12-00 \mathrm{~h}$ & $27 / 12-00 \mathrm{~h}$ & $27 / 12-00 \mathrm{~h}$ & $27 / 12-00 \mathrm{~h}$ \\
\hline $28 / 12 / 2013$ & $43-45$ & moy : 2,23 & $\mathrm{moy}: 2,76$ & moy $: 3.53$ & moy $: 3.85$ & moy: 3.49 \\
\hline tempête & $28 / 12$ & $\max : 3,48$ & $\max : 3,88$ & $\max : 4.61$ & $\max : 5.07$ & $\max : 4.73$ \\
\hline Erich & $48-52$ & $27 / 12-13 \mathrm{~h}$ & $27 / 12-12 \mathrm{~h}$ & $27 / 12-12 \mathrm{~h}$ & $27 / 12-11 \mathrm{~h}$ & $27 / 12-11 \mathrm{~h}$ \\
\hline & & moy $: 3,53$ & moy : 4,35 & moy : 5.34 & moy : 5.07 & moy : 4.14 \\
\hline & & $\max : 5,47$ & $\max : 6.03$ & $\max : 6.62$ & $\max : 6.92$ & $\max : 5.68$ \\
\hline & & $28 / 12-01 \mathrm{~h}$ & $28 / 12-01 \mathrm{~h}$ & $28 / 12-01 \mathrm{~h}$ & $28 / 12-01 \mathrm{~h}$ & $28 / 12-01 \mathrm{~h}$ \\
\hline & & moy : 2,91 & moy : 3.56 & moy $: 4.20$ & moy $: 4.06$ & moy $: 3.54$ \\
\hline & & $\max : 4,44$ & $\max : 4,75$ & $\max : 5.10$ & $\max : 5.64$ & $\max : 5.06$ \\
\hline $01 / 01 / 2014$ & 01/01: & $01 / 01-06 h$ & $01 / 01-05 \mathrm{~h}$ & $01 / 01-05 \mathrm{~h}$ & $01 / 01-03 \mathrm{~h}$ & $01 / 01-03 \mathrm{~h}$ \\
\hline $02 / 01 / 2014$ & $96-100$ & moy : 2,80 & moy : 3,68 & moy $: 4,49$ & moy : 4,85 & moy $=4,24$ \\
\hline (sans nom) & 02/01: & $\max : 5,20$ & $\max : 5.49$ & $\max : 5,89$ & $\max : 7.41$ & $\max : 6,02$ \\
\hline & $104-107$ & $01 / 01-18 h$ & $01 / 01-17 \mathrm{~h}$ & $01 / 01-17 \mathrm{~h}$ & $01 / 01-16 h$ & $01 / 01-16 \mathrm{~h}$ \\
\hline & & moy : 3,02 & moy : 3,93 & moy : 4,79 & moy : 5,42 & moy : 5,36 \\
\hline & & $\max : 5,55$ & $\max : 6,01$ & $\max : 6,45$ & $\max : 8,00$ & $\max : 7,23$ \\
\hline & & $02 / 01-06 \mathrm{~h}$ & $02 / 01-05 \mathrm{~h}$ & $02 / 01-05 \mathrm{~h}$ & $02 / 01-04 \mathrm{~h}$ & $02 / 01-04 \mathrm{~h}$ \\
\hline & & moy : 2,93 & moy : 3,73 & moy $: 4,14$ & moy : 4,53 & moy $: 4,51$ \\
\hline & & $\max : 5,00$ & $\max : 5,36$ & $\max : 5.38$ & $\max : 7,14$ & $\max =6,22$ \\
\hline $03 / 01 / 2014$ & $03 / 01:$ & $03 / 01-07 \mathrm{~h}$ & $03 / 01-06 \mathrm{~h}$ & $03 / 01-06 \mathrm{~h}$ & $03 / 01-05 \mathrm{~h}$ & $03 / 01-05 \mathrm{~h}$ \\
\hline $04 / 01 / 2014$ & 108 & moy : 2,48 & moy : 3,10 & moy $: 3.88$ & moy $=4.18$ & moy $: 3.98$ \\
\hline (sans nom) & 04/01: & $\max : 4,41$ & $\max : 4,88$ & $\max =5.26$ & $\max =6.50$ & $\max : 5.19$ \\
\hline & $106-103$ & $03 / 01-20 \mathrm{~h}$ & $03 / 01-19 \mathrm{~h}$ & $03 / 01-19 h$ & 03/01-18h & $03 / 01-17 \mathrm{~h}$ \\
\hline & & moy : 3,72 & moy : 4,92 & moy : 5.91 & moy $: 6.26$ & moy : 5.33 \\
\hline & & $\max : 6,76$ & $\max : 7,50$ & $\max : 7.92$ & $\max : 9.13$ & $\max : 7.43$ \\
\hline & & $04 / 01-08 \mathrm{~h}$ & $04 / 01-07 \mathrm{~h}$ & $04 / 01-07 \mathrm{~h}$ & $04 / 01-06 \mathrm{~h}$ & $04 / 01-06 \mathrm{~h}$ \\
\hline & & moy : 2,68 & moy : 3,56 & moy $: 4.30$ & moy $: 4.94$ & moy: 5.37 \\
\hline & & $\max : 4,98$ & $\max : 5,51$ & $\max =5.70$ & $\max =7.77$ & $\max : 7.18$ \\
\hline $06 / 01 / 2014$ & $06 / 01:$ & $06 / 01-09 \mathrm{~h}$ & $06 / 01-08 \mathrm{~h}$ & $06 / 01-09 \mathrm{~h}$ & $06 / 01-07 \mathrm{~h}$ & $06 / 01-07 \mathrm{~h}$ \\
\hline $07 / 01 / 2014$ & $88-82$ & moy $: 2,96$ & moy $: 3,81$ & moy $: 4.71$ & moy $: 5.12$ & moy $: 4.97$ \\
\hline (sans nom) & 07/01: & $\max : 5,35$ & $\max : 6,01$ & $\max =6.64$ & $\max =7.62$ & $\max : 6.62$ \\
\hline & $74-68$ & $06 / 01-22 \mathrm{~h}$ & $06 / 01-21 \mathrm{~h}$ & $06 / 01-21 \mathrm{~h}$ & 06/01-20h & 06/01-20h \\
\hline & & moy : 3,39 & moy : 4,49 & moy : 5.69 & moy : 6.29 & moy : 5,90 \\
\hline & & $\max : 6,34$ & $\max : 7,18$ & $\max : 7.98$ & $\max : 9.63$ & $\max : 8.22$ \\
\hline & & $07 / 01-10 \mathrm{~h}$ & $07 / 01-09 \mathrm{~h}$ & $07 / 01-09 \mathrm{~h}$ & 07/01-OBh & $07 / 01-08 \mathrm{~h}$ \\
\hline & & moy : 3,11 & moy : 4.09 & may : 5.21 & moy : 5.53 & moy: 5.32 \\
\hline & & $\max : 5,76$ & $\max : 6,47$ & $\max =7.16$ & $\max : 8.47$ & $\max : 7.28$ \\
\hline $26 / 01 / 2014$ & 26/01: & $26 / 01-13 \mathrm{~h}$ & $26 / 01-12 \mathrm{~h}$ & $26 / 01-13 \mathrm{~h}$ & $26 / 01-13 \mathrm{~h}$ & $26 / 01-11 \mathrm{~h}$ \\
\hline $27 / 01 / 2014$ & $46-47$ & moy : 2,85 & moy : 3,70 & moy : 5.30 & moy: 5.05 & moy: 3.09 \\
\hline $28 / 01 / 2014$ & 27/01: & $\max : 4,22$ & $\max : 4,90$ & $\max : 7.52$ & $\max : 7.73$ & $\max : 5.00$ \\
\hline (sans nom) & 51.57 & $27 / 01-02 \mathrm{~h}$ & $27 / 01-01 \mathrm{~h}$ & 27/01-01h & $27 / 01-00 \mathrm{~h}$ & $27 / 01-00 \mathrm{~h}$ \\
\hline & 28/01: & moy : 3,86 & moy $: 4,57$ & moy : 5.79 & moy : 5.35 & moy $: 3.57$ \\
\hline & $64-72$ & $\max : 5.39$ & $\max : 5.77$ & $\max : 7.88$ & $\max : 8.19$ & $\max : 5.32$ \\
\hline & & 27/01-15h & 27/01-14h & $27 / 01-14 h$ & $27 / 01-14 h$ & $27 / 01-13 \mathrm{~h}$ \\
\hline & & moy : 3,78 & moy $: 4,75$ & moy: 5.90 & moy: 5.70 & moy : 3.94 \\
\hline & & $\max : 5,45$ & $\max : 6,16$ & $\max : 7.68$ & $\max : 8.05$ & $\max : 6.19$ \\
\hline & & 28/01-03h & $28 / 01-02 h$ & $28 / 01-02 h$ & 28/01-01h & 28/01-01h \\
\hline & & moy : 4,21 & moy : $\mathbf{5 , 3 0}$ & moy : 6.41 & moy : 6.07 & moy : 4.35 \\
\hline & & $\max : 6,20$ & $\max : 6,87$ & $\max : 8.10$ & $\max : 8.67$ & $\max : 6.74$ \\
\hline & & $28 / 01-16 \mathrm{~h}$ & $28 / 01-15 h$ & $28 / 01-15 h$ & $28 / 01-14 h$ & $28 / 01-14 h$ \\
\hline & & moy : 3,16 & moy : 4,06 & moy: 4.77 & moy $: 4.79$ & moy: 3.71 \\
\hline & & $\max : 4,58$ & $\max : 5,31$ & $\max : 6.19$ & $\max : 7.03$ & $\max : 5.66$ \\
\hline $01 / 02 / 2014$ & 01/02: & $01 / 02-07 \mathrm{~h}$ & $01 / 02-06 \mathrm{~h}$ & $01 / 02-06 \mathrm{~h}$ & $01 / 02-05 \mathrm{~h}$ & $01 / 02-05 \mathrm{~h}$ \\
\hline $02 / 02 / 2014$ & $113-114$ & moy : 3.57 & moy : 4.39 & moy $: 5.15$ & moy: 5.13 & moy: 4.24 \\
\hline (sans nom) & $02 / 02:$ & $\max : 5.49$ & $\max : 6.01$ & $\max : 6.70$ & $\max : 8.23$ & $\max : 5.87$ \\
\hline & $113-110$ & $01 / 02-19 h$ & $01 / 02-19 h$ & $01 / 02-18 \mathrm{~h}$ & $01 / 02-17 \mathrm{~h}$ & $01 / 02-17 \mathrm{~h}$ \\
\hline & & moy : 4,23 & moy : 5,39 & moy : 6.61 & moy : 6.62 & moy : 5.09 \\
\hline & & $\max : 6,59$ & $\max : 7,30$ & $\max : 9.04$ & $\max : 10.71$ & $\max : 7.48$ \\
\hline & & $02 / 02-08 \mathrm{~h}$ & $02 / 02-07 \mathrm{~h}$ & $02 / 02-07 \mathrm{~h}$ & $02 / 02-06 \mathrm{~h}$ & $02 / 02-05 \mathrm{~h}$ \\
\hline & & moy : 3.72 & moy : 4.75 & moy $: 5.50$ & moy: 5.63 & moy : 4.45 \\
\hline & & $\max : 5.48$ & $\max : 6.51$ & $\max =7.54$ & $\max : 8.88$ & $\max : 6.64$ \\
\hline $04 / 02 / 2014$ & 04/02: & $04 / 02-21 \mathrm{~h}$ & $04 / 02-20 h$ & $04 / 02-21 \mathrm{~h}$ & $04 / 02-20 \mathrm{~h}$ & $04 / 02-19 h$ \\
\hline $05 / 02 / 2014$ & $94-87$ & moy : 2,29 & moy : 2,74 & moy : 3.64 & moy :5.19 & moy : 4.93 \\
\hline tempête & $05 / 02$ & $\max : 3.58$ & $\max : 4,13$ & $\max : 5.93$ & $\max : 7.30$ & $\max : 6.07$ \\
\hline Petra & $79-71$ & $05 / 02-10 \mathrm{~h}$ & $05 / 02-09 h$ & $05 / 02-09 h$ & $05 / 02-08 \mathrm{~h}$ & $05 / 02-07 \mathrm{~h}$ \\
\hline & & moy : 3.44 & moy : 4,45 & moy : 6.12 & moy : 6.89 & moy : 6.44 \\
\hline & & $\max : 6,50$ & $\max : 7,66$ & $\max : 9.25$ & $\max : 10.41$ & $\max : 8.66$ \\
\hline & & $05 / 02-22 h$ & $05 / 02-21 \mathrm{~h}$ & $05 / 02-21 \mathrm{~h}$ & $05 / 02-20 \mathrm{~h}$ & $05 / 02-20 \mathrm{~h}$ \\
\hline & & moy : 4,03 & moy : 5,21 & moy $=6.38$ & moy : 6.73 & moy : 6.48 \\
\hline & & $\max : 6,80$ & $\max : 7,90$ & $\max : 8.49$ & $\max : 9.88$ & $\max : 8.89$ \\
\hline $06 / 02 / 2014$ & $06 / 02$ & 06/02-10h & 06/02-09h & $06 / 02-09 \mathrm{~h}$ & $06 / 02-08 \mathrm{~h}$ & 06/02-08h \\
\hline $07 / 02 / 2014$ & $63-55$ & moy : 2,83 & moy : 3,65 & moy : 4,24 & moy : 4,34 & moy : 4,08 \\
\hline
\end{tabular}


Les hauteurs moyennes et maximum des houles sont obtenues à partir de modèles issus du système d'observations et de prédictions PREVIMER (http://previmer.fr).

Wave heights (average and maximum) are obtained from wave modelling produced in a frame of PREVIMER observations and forecasts system (http://previmer.fr).

5La période d'activité tempétueuse a véritablement commencé avec la tempête Dirk du 23 décembre 2013, et s'est terminée par la tempête Christine du 3 mars 2014. La plupart de ces épisodes ont duré plusieurs jours, cela a eu pour conséquence de générer des fortes houles qui ont perduré même après le passage de la perturbation. Pour chacun d'entre eux, on note que de façon générale les hauteurs de houle diminuent au fur et à mesure que l'on progresse d'ouest (fenêtre ouest Finistère) en est (fenêtres centrées sur les Côtes d'Armor, et, dans une moindre mesure, le sud Finistère). Ceci est principalement lié aux phénomènes d'amortissement de la houle sur la plateforme continentale. Certaines tempêtes ont été combinées à une marée de vive-eau. C'est généralement dans ces conditions que les phénomènes d'érosion du trait de côte sont les plus importants. Ainsi, entre le 23 et le 28 décembre, les tempêtes Dirk et Erich ont été peu morphogènes car elles sont survenues en période de morte-eau avec des houles peu énergétiques caractérisées par des hauteurs moyennes et maximales respectivement comprises entre $3,51 \mathrm{~m}$ et $5,96 \mathrm{~m}$, et $5,80 \mathrm{~m}$ et $8,30 \mathrm{~m}$ suivant les secteurs. À l'inverse, les deux tempêtes survenues au début du mois de janvier ont été combinées à des marées de vive-eau, notamment celle du 3 et 4 janvier durant laquelle les coefficients de marée étaient supérieurs à 105. De plus, les hauteurs de houles moyennes et maximales plus élevées comprises respectivement entre $3,72 \mathrm{~m}$ et $6,26 \mathrm{~m}$, et $6,76 \mathrm{~m}$ et $9,13 \mathrm{~m}$, ont renforcé le caractère morphogène de cet épisode. Il en a été de même pour la tempête du 1 et 2 février durant laquelle les coefficients de marée ont été supérieurs à 110, et pour la tempête Petra du 4 et 5 février durant laquelle les coefficients de marée étaient encore supérieurs à 90. Qui plus est, pour ces deux épisodes, les hauteurs de houles, notamment maximales, ont atteintrespectivement 10,71 $\mathrm{m}$ et 10,41 $\mathrm{m}$ au large de l'ouest du Finistère. Les trois tempêtes Qumaira, Ruth et Ulla qui ont suivi sont survenues en période de morte-eau, notamment celle du 6 et 7 février (Qumaira) et du 8 et 9 février (Ruth) pour lesquelles les hauteurs moyennes et maximales de houle étaient respectivement d'environ 2,78 $\mathrm{m}$ et 4,11 m, et $4,97 \mathrm{~m}$ et $7,74 \mathrm{~m}$, Ruth ayant été plus énergétique. La tempête Ulla a été marquée par un renforcement des conditions d'agitation (comprises 8,01 m et 10,38 m suivant les secteurs), mais les coefficients étaient encore faibles (de 78 à 86 du 14 au 15 février). Les deux derniers épisodes du début du mois de mars ont été de nouveau combinés à des fortes marées de viveeau (coefficient $>110$ ), notamment la tempête Christine pour laquelle les maxima de hauteurs de houle ont atteint 12,63 $\mathrm{m}$ au large de l'ouest du Finistère.

\section{$\underline{\text { 3. Les sites d'étude }}$}

6Les sites d'étude se répartissent le long des trois façades littorales nord, ouest et sud de la Bretagne (fig. 2). Ils correspondent à des sites ateliers sur lesquels des suivis topomorphologiques sont menés depuis plusieurs années dans le cadre de différents programmes d'observation. Le premier d'entre eux appelé Observatoire du Domaine Côtier (ODC-IUEM), concerne un projet lancé en 2002 dans le cadre des activités scientifiques de l'Institut Universitaire Européen de la Mer (IUEM). Cet observatoire couvre une trentaine de sites répartis sur le pourtour de la Bretagne, qui sont suivis avec une fréquence variable suivant les secteurs (Suanez et al., 2012a). Le second projet dans lequel s'inscrivent nos suivis consiste en un système d'observation national qui couvre l'ensemble des côtes de la France 
métropolitaine et des départements d'outre-mer; il s'agit du SOERE trait de côte, aménagements littorau (Système d'Observation et d'Expérimentation à long terme pour la Recherche en Environnement), créé en 2010 et financé par AllEnvi (l'alliance nationale de recherche pour l'environnement qui regroupe le Ministère de l'Enseignement Supérieur et Recherche et la Direction Générale Recherche et Innovation). Le troisième programme d'observation concerne les mesures topo-morphologiques effectuées dans le cadre du projet de recherche scientifique COCORISCO (Connaissance et Compréhension des Risques Côtier), qui a été lancé en 2011, et financé sur 3 ans par l'Agence Nationale de la Recherche (ANR). Dans le cadre de ce projet, un suivi morphologique à haute fréquence a été lancé sur plusieurs plages du sud Finistère, notamment sur les cordons littoraux de Penmarc'h et de Treffiagat-Léchiagat.

Fig. 2 - Carte de localisation des différents sites d'étude.

Fig. 2 - Location map of study sites.

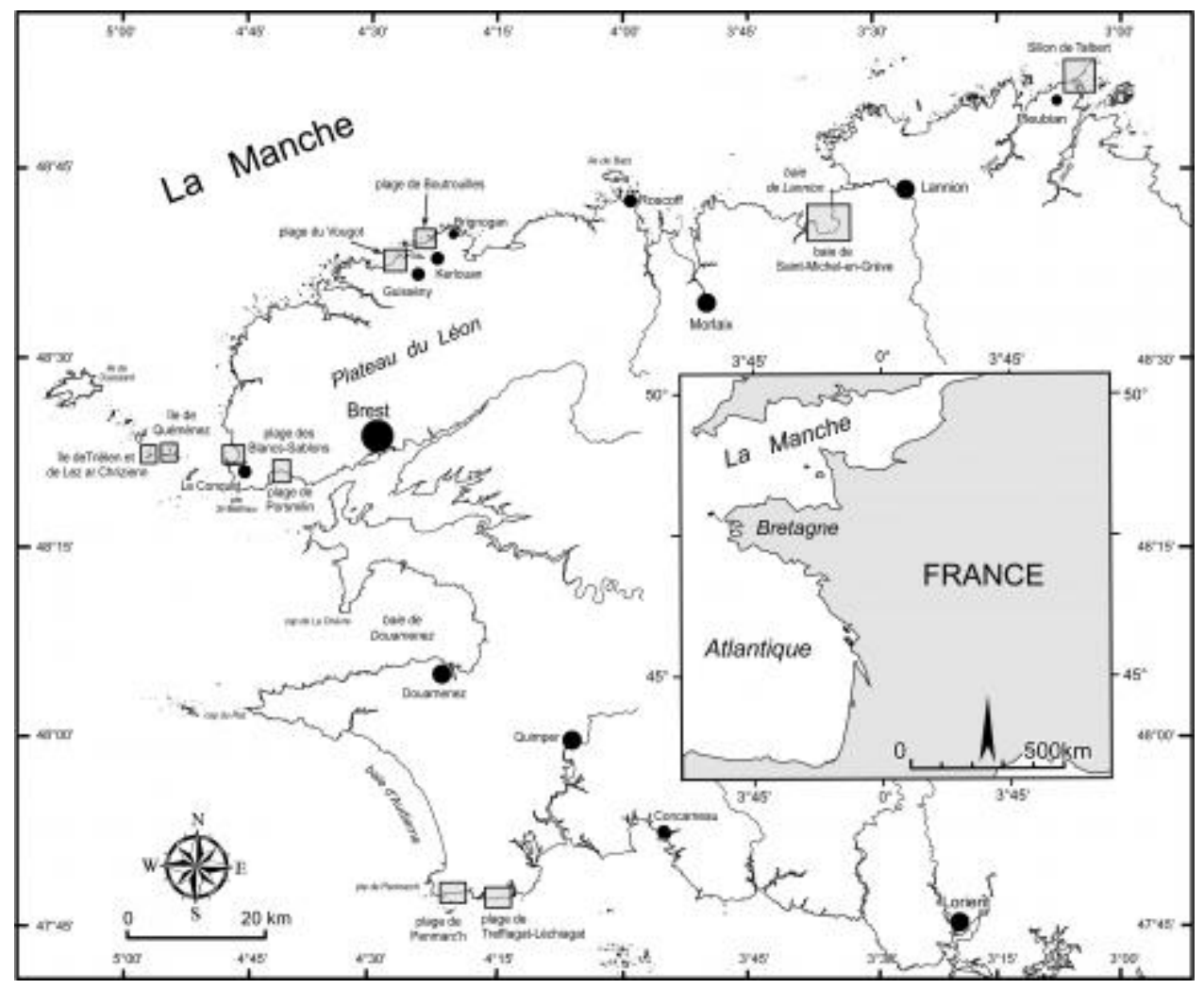

Agrandir Original (jpeg, 1,4M)

7Au nord de la Bretagne, dans le département des Côtes d'Armor, deux sites ont été retenus. Le premier correspond à la baie de Saint-Michel-en-Grève constituée d'une vaste plage sableuse de fond de baie formée de petits cordons dunaires adossés à des ouvrages de défense côtière (Suanez et Stéphan, 2011). Le second concerne le Sillon de Talbert correspondant quant à lui à une flèche de galets à pointe libre longue de plus de 3,5 km de distance (Stéphan et al., 2012). Ces deux sites font l'objet d'un suivi morphosédimentaire annuel commencé respectivement en février 1998 et en octobre 2002. Sur la côte nord du département du Finistère, les sites d'étude sont constitués de grandes plages sableuses associées à de grands complexes dunaires fermant des zones basses situées en arrière comme la plage du Vougot à Guissény, et de Boutrouilles à Kerlouan (Suanez et al., 2012b ; Suanez et Cariolet, 2010). Ces deux plages sont suivies respectivement depuis les mois de juillet 2004 à une fréquence 
mensuelle et de novembre 2006 à une fréquence annuelle. À l'ouest du Finistère, les sites correspondent d'une part aux îles de Triélen, de Lez ar Chrizienn et de Quéménez dans l'archipel de Molène (fig. 2), en partie formées de matériaux cohérents correspondant à des plages pléistocènes soulevées et/ou de formations périglaciaires de type head, à l'arrière desquelles se sont construit des queues de comète constituées de galets (Suanez et al., 2011) ; d'autre part, à la plage sableuse de fond d'anse des Blancs Sablons située au nord du Conquet, et à celle de Porsmilin qui correspond à une plage de fond de ria localisée dans l'anse de Bertheaume (Dehouck et al., 2009). L'ensemble de ces sites font l'objet d'un suivi morphosédimentaires qui a débuté dans le courant de l'année 2002 à une fréquence annuelle dans l'archipel de Molène, et mensuelle pour les plages des Blancs Sablons et de Porsmilin. Enfin, pour le sud Finistère, les deux grands cordons sableux de Penmarc'h et de TreffiagatLéchiagat, formés de dunes en partie artificialisées barrant des zones basses arrière-littorales, constituent les deux zones d'étude principales (Bodéré et al., 1993). Le travail d'observation réalisé sur ces deux plages est plus récent, il a débuté au mois de juin 2011 à une fréquence trimestrielle.

\section{Méthode et données}

\subsection{Analyse des conditions météomarines et hydrodynamiques}

8L'analyse des conditions météomarines repose sur les données de vent et de pression barométrique enregistrées à Brignogan pour la côte nord de la Bretagne, au phare du Stiff à Ouessant pour la pointe occidentale du Finistère, et à Penmarc'h pour la côte sud bretonne (fig. 3). Si l'on s'en tient à la définition de l'Organisation Météorologique Mondiale, le seuil de tempête est atteint lorsque la vitesse du vent sur l'océan atteint entre 44 et 50 nœuds, soit en moyenne 48 nœuds, soit approximativement $25 \mathrm{~m} / \mathrm{s}$. Toutefois, l'effet du frottement continental justifie le choix d'un seuil inférieur sur terre compris entre 18 et $20 \mathrm{~m} / \mathrm{s}$ (Trzpit, 1977). Ces valeurs seuil ont été retenues pour mieux caractériser les conditions météorologiques agissant localement lors des épisodes de tempête les plus morphogènes.

Fig. 3 - Caractéristiques de la houle basée sur les enregistrements réalisés au large du Finistère par la bouée Datawell des Pierres Noires, et marées observées pour la côte nord, ouest et sud de la Bretagne, entre les mois de décembre 2013 et mars 2014. Fig. 3 - Wave characteristics based on waverider measurements achieved off western Brittany peninsula (Pierres Noires buoy), and tide measurements achieved for the North, West and South Brittany coast, between December 2013 and April 2014. 


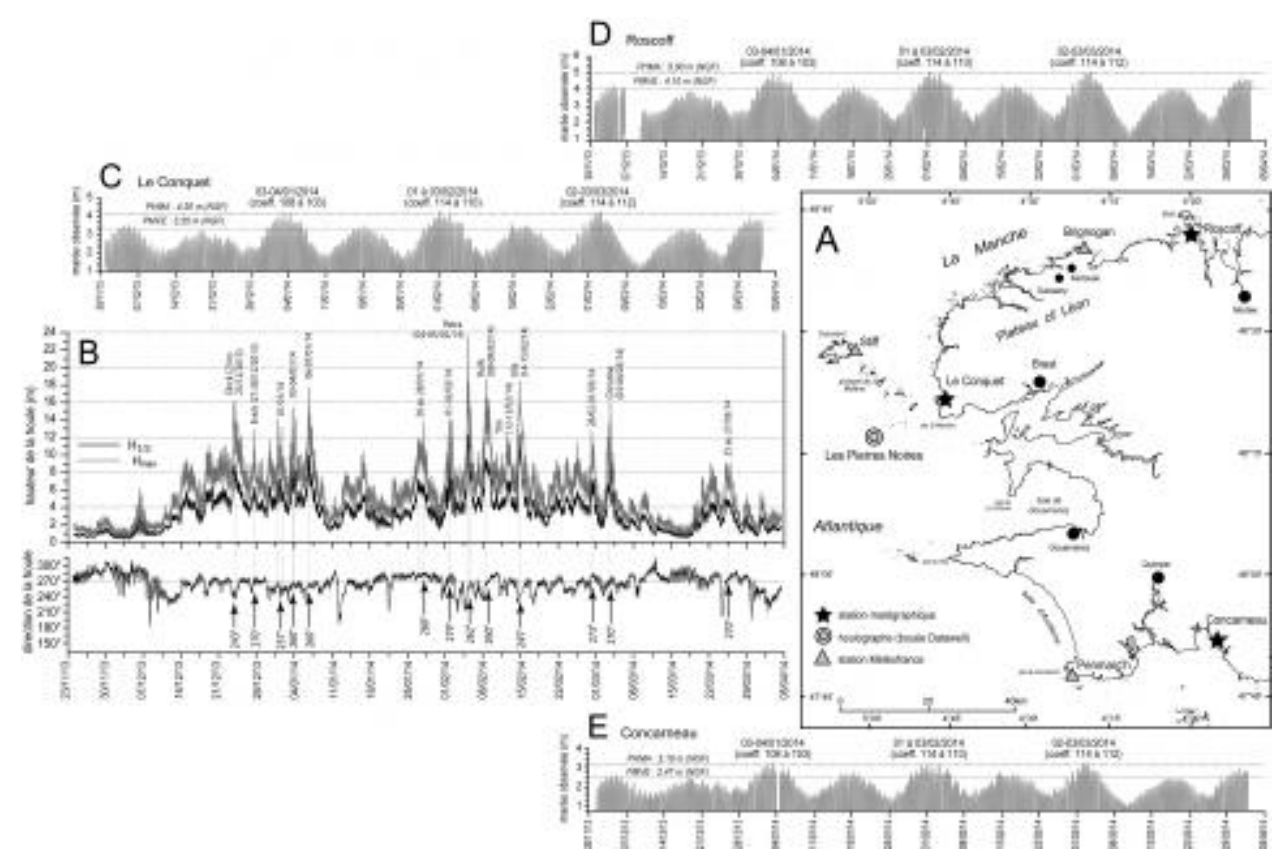

$\underline{\text { Agrandir }} \underline{\text { Original (jpeg, 2,7M) }}$

A : localisation de la bouée Datawell des Pierres Noires et des marégraphes de Roscoff, du Conquet et de Concarneau; B : hauteurs et directions de la houle; marée observée au Conquet (C), à Roscoff (D), à Concarneau (E), comparée au niveau des pleines mers de viveeau et au niveau des plus hautes mers astronomiques.

A: Location map of Pierres Noires wave buoy, and Roscoff, Le Conquet, and Concarneau tide gauge stations; B: Wave height and direction; Observed tide measurement at Le Conquet (C), Roscoff (D), Concarneau (E) compared with Mean High Water Spring and High Astronomic Tide levels for each location.

9L'analyse de la houle repose sur les enregistrements effectués à l'ouest de la péninsule bretonne par une bouée Datawell directionnelle du projet PREVIMER intégrée au réseau national du CEREMA. Il s'agit de la bouée des Pierres Noires située au sud de l'archipel de Molène, à $10 \mathrm{~km}$ de la côte, et à une profondeur de $60 \mathrm{~m}$ (coordonnées : 48 $17,420^{\prime} \mathrm{N}$; $4^{\circ} 58,100^{\prime} \mathrm{W}$; fig. 3A). Cette bouée est généralement abritée des vagues d'ouest à nord-ouest et subit une forte modulation des hauteurs par des courants autour de l'île de Ouessant avec une amplification locale à marée haute (Ardhuin et al., 2012). L'analyse de ces données montre que pour la douzaine d'épisodes tempétueux inventoriés précédemment, les hauteurs maximum de houle ont été supérieures à $12 \mathrm{~m}$, et pour la moitié d'entre eux $\geq$ à $16 \mathrm{~m}$ (fig. 3B). La valeur maximum concerne la tempête Petra du 5 février durant laquelle une hauteur maximum d'environ 23,5 $\mathrm{m}$ a été enregistrée. En matière de hauteurs significatives, les deux tempêtes Petra et Ulla du mois de février ont été marquées par des houles supérieures à $12 \mathrm{~m}$ dont la période de retour est comprise entre 10 et 30 ans. Pour six d'entre elles, notamment la tempête Dirk du 24décembre, celles du 3-4 et 6-7 janvier, du 1-2 février, et des tempêtes Ruth du 8-9février et Christine du 3 mars, les hauteurs significatives ont atteint entre $8 \mathrm{~m}$ et $9,9 \mathrm{~m}$, ce qui correspond à des périodes de retour comprises entre 1 et 10 ans. Toutefois, il est important de noter que ces périodes de retour ont peu de représentativité statistique car la période d'échantillonnage de 6,15 années reste trop courte, et sous-estime de ce fait la réalité (CEREMA, 2014). En ce qui concerne les directions, ce sont essentiellement des houles d'ouest qui ont agi durant ces tempêtes. Les directions de provenance de la houle incidente au pic s'échelonnent entre $243^{\circ}(\mathrm{O}-\mathrm{SO})$ pour la tempête 
Dirk du 23 décembre 2013, et $280^{\circ}$ (O-NO) pour la tempête du 26 au 28 janvier, et du 1 2 février 2014. Pour autant, c'est essentiellement la direction $260^{\circ}-270^{\circ}$ qui domine, soit une provenance principale d'ouest (fig. 3B).

10L'analyse des niveaux d'eau repose quant à elle sur les enregistrements marégraphiques des stations de Roscoff pour la côte nord de la Bretagne, du Conquet pour la côte occidentale, et de Concarneau pour la côte sud (fig. 3). Ces données montrent que pour trois épisodes, les niveaux de la marée observée ont été supérieurs aux niveaux des plus hautes marées astronomiques (PHMA). Il s'agit des trois grandes marées de vive-eau du 3-4 janvier, du 1 au 3 février, et du 2-3 mars, durant lesquelles les coefficients de marée ont été supérieurs à 110 . Une analyse des surcotes a également été réalisée sur l'ensemble de la période d'observation (fig. 4). Pour les trois séries marégraphiques, ces résultats ont été comparés aux niveaux maximum calculés pour différentes périodes de retour dans le cadre d'une étude réalisée par les CETMEF et le CETE Méditerranée (Perherin et al., 2013; tab. 2). De façon générale, c'est au mois de février que les surcotes les plus fortes, associées à des périodes de retour comprises entre 20 et 50 ans, ont été enregistrées, notamment à Roscoff et au Conquet (fig. 4A et 4B). Ainsi, durant la tempête Ulla, une surcote d'occurrence centennale de $0,97 \mathrm{~m}$ a été observée au Conquet (le 14 février 2014 à 17 h). Pour la même période, les niveaux mesurés à Concarneau montrent des hauteurs moins importantes, associées tout de même à des périodes de retour comprises entre 5 et 20 ans (fig. 4C). Au marégraphe de Concarneau, la plus forte surcote de l'hiver a été enregistrée le 24 décembre 2013 lors de la tempête Dirk (fig. $4 \mathrm{C}$ ), soit $0,82 \mathrm{~m}$, ce qui correspond à un niveau d'occurrence cinquantennale à centennale.

Fig. 4 - Analyse des surcotes enregistrées par les marégraphes de Roscoff pour la côte nord de la Bretagne (A), du Conquet pour la pointe occidentale (B), et de Concarneau pour la côte méridionale $(\mathrm{C})$, comparées aux niveaux de surcote maximum pour des périodes de retour de $5,20,50$ et 100 ans.

Fig. 4 - Surge analysis recorded by tide gauge station of Roscoff for North Brittany coast (A), of Conquet for Western Brittany coast (B), and Concarneau for South Brittany coast (C), compared to maximum surge levels for 5-yr, 20-yr, 50-yr, and 100-yr return periods.

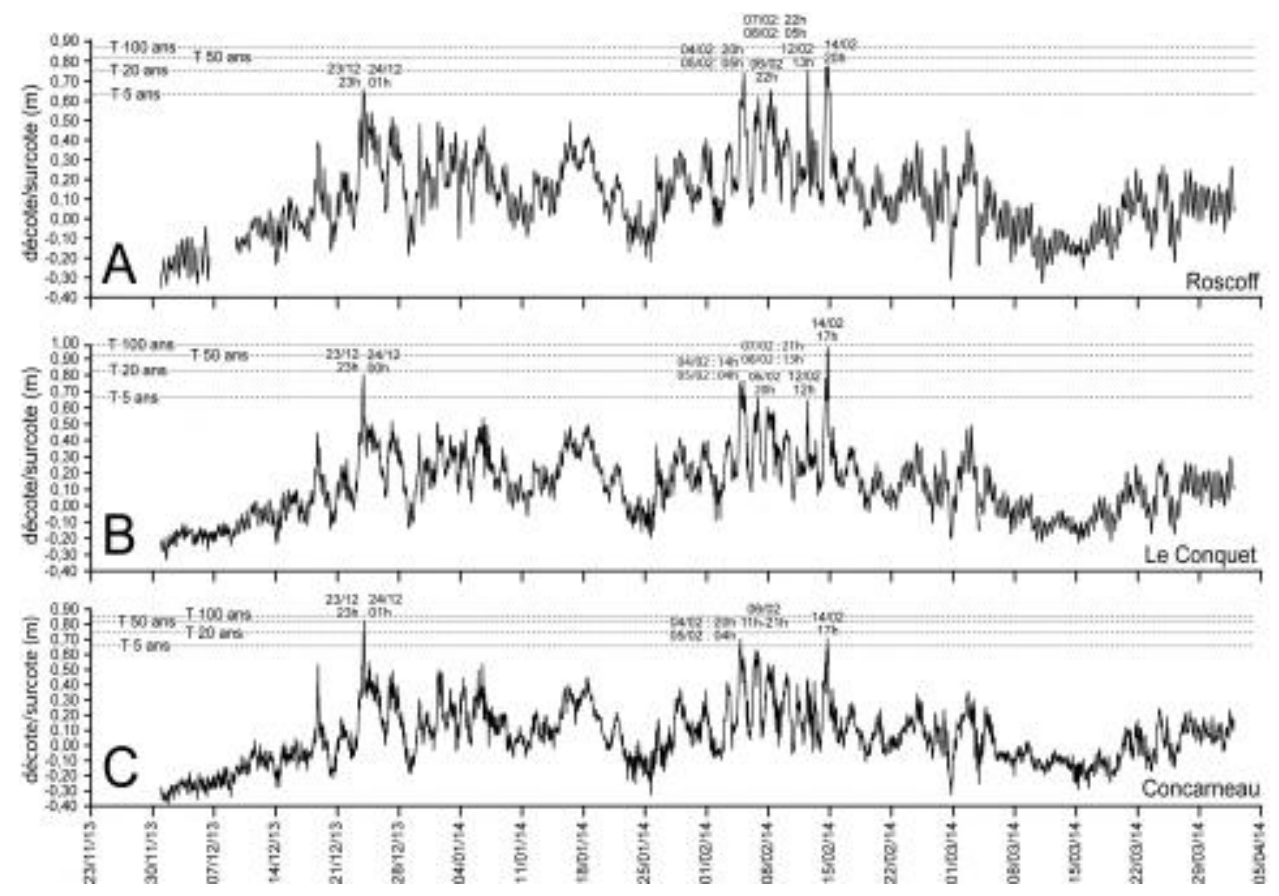


Tab. 2 - Estimation des surcotes extrêmes calculées selon la loi d'ajustement GPD (loi de Distribution Généralisée de Pareto) à partir des enregistrements marégraphiques du Conquet, de Roscoff et de Concarneau.

Tab. 2 - Estimation of extreme surges calculated using the GPD fit (generalized Pareto distribution) from the tide gauge records of Conquet, Roscoff and Concarneau.

\begin{tabular}{|c|c|c|c|c|c|c|}
\hline Période de retour (années) & 5 & 10 & 20 & 50 & 100 & 10000 \\
\hline Surcote de PM (m) & 0,67 & 0,75 & 0,83 & 0,93 & 1,02 & 1,31 \\
\hline Intervalle de confiance ( $m$ ) & $0,64-0,70$ & $0,71-0,79$ & $0,77-0,89$ & $0,85-1,02$ & $0,90-1,13$ & $1,07-1,56$ \\
\hline \multicolumn{7}{|c|}{ Roscoff (longueur de l'échantillon : 36,4 années - 1973 a 2012) } \\
\hline Période de retour (années) & 5 & 10 & 20 & 50 & 100 & 1000 \\
\hline Surcote de PM (m) & 0,63 & 0,69 & 0,75 & 0,82 & 0,87 & 1,04 \\
\hline Intervalle de confiance (m) & $0,60-0,66$ & $0,66-0,72$ & $0,70-0,79$ & $0,76-0,88$ & $0,80-0,95$ & $0,90-1,17$ \\
\hline \multicolumn{7}{|c|}{ Concarneau (longueur de l'ćchantillon : 12,6 années - 1999 à 2012) } \\
\hline Période de retour (années) & 5 & 10 & 20 & 50 & 100 & 1000 \\
\hline Surcote de PM (m) & 0,64 & 0,70 & 0,75 & 0,81 & 0,86 & 0,99 \\
\hline Intervalle de confiance ( $\mathrm{m}$ ) & $0,59-0,68$ & $0,63-0,76$ & $0,67-0,83$ & $0,30-0,93$ & $0,72 \cdot 1,00$ & $0,75-1,22$ \\
\hline
\end{tabular}

\section{Agrandir $\underline{\text { Original (ipeg, 524k) }}$}

Source : Perherin et al., 2013.

Source: Perherin et al., 2013.

11Une analyse plus détaillée des surcotes a également été réalisée en distinguant les surcotes instantanées des niveaux d'eau extrêmes enregistrés pour chacun des épisodes tempétueux qui ont été listés plus haut (tab. 3). L'observation de ces données montre qu'il y a eu très peu de tempêtes durant lesquelles les surcotes instantanées maximum ont été combinées à une pleine mer. Pour les trois marégraphes, cette concomitance s'observe lors des épisodes du début du mois de février, les 4,7 et 9 février, et dans une moindre mesure le 2 mars. Nous pouvons également voir que le 4 février et le 2 mars, la surcote maximum instantanée a été combinée à une forte marée, notamment la marée de vive-eau du 2 mars (coefficient 114-115). Cela a eu pour effet de générer des hauts niveaux d'eau supérieurs aux PMVE (pleine mer de vive-eau) et/ou PHMA (plus haute marée astronomique). Toutefois, on peut noter que dans l'ensemble, les surcotes maximum instantanées pour des périodes de retour comprises entre 50 et 100 ans, sont survenues au moment d'une basse mer et/ou d'une marée de morte-eau. C'est le cas par exemple des surcotes mesurées respectivement le 14 février au Conquet à $19 \mathrm{~h}$ et à Roscoff à $20 \mathrm{~h}$, ou à Concarneau le 24 décembre à $01 \mathrm{~h}$.

Tab. 3 - Niveaux d'eau maximum et surcotes instantanées enregistrés par les marégraphes de Roscoff, du Conquet, et de Concarneau, pour les épisodes tempétueux survenus durant l'hiver 2013-2014.

Tab. 3 - Maximum water level and instantaneous surge calculated using records of Conquet, Concarneau and Roscoff tide gauge stations during winter 2013-14 storm events. 


\begin{tabular}{|c|c|c|c|c|c|c|c|}
\hline \multirow[b]{2}{*}{ maregraphe } & \multicolumn{4}{|c|}{ niveau d'eau maximum de pleine mer } & \multicolumn{3}{|c|}{ surcote maximum instantanée } \\
\hline & $\begin{array}{l}\text { have. } \\
\text { max }\end{array}$ & index & jour et heure & $\begin{array}{l}\text { surcose } \\
\text { a la PM }\end{array}$ & $\begin{array}{l}\text { surcose } \\
\text { instant. }\end{array}$ & jour et heure & $\begin{array}{c}\text { niveau } \\
\text { d'eau } \\
\text { associd }\end{array}$ \\
\hline \multirow{31}{*}{$\begin{array}{l}\text { Le Conquet } \\
\text { PMVE : } 6,85 \\
\text { PHMA : } 7,69\end{array}$} & 6.30 & $\mathrm{H}<\mathrm{PMVE}$ et PHMA & $23 / 12 / 2013: 20 \mathrm{~h}$ & 0.58 & 0.80 & $23 / 12 / 2013: 23 h$ & 4.63 \\
\hline & 6.20 & $\mathrm{H}<\mathrm{PMVE}$ et PHMA & 24/12/2013: Doh & 0,36 & 0,56 & $24 / 12 / 2013: 00 h$ & 3,12 \\
\hline & 5.95 & $H<$ PMVE et PHMA & $25 / 12 / 2013: 09 h$ & 0,35 & 0.47 & $25 / 12 / 2013: 03 h$ & 3.04 \\
\hline & 5.82 & $H<P M V E$ et PHMA & $27 / 12 / 2013: 11 \mathrm{~h}$ & 0,36 & 0,52 & $27 / 12 / 1013: 05 h$ & 3,24 \\
\hline & 5.81 & $H<$ PMVE et PHMA & $28 / 12 / 2013=12 \mathrm{~h}$ & 0.17 & 0,33 & $28 / 12 / 2013: 06 h$ & 2,88 \\
\hline & 7,32 & PMVE $<H<P H M A$ & $01 / 01 / 2014: 16 \mathrm{~h}$ & 0,33 & 0.51 & $01 / 01 / 2014: 10 h$ & 1,44 \\
\hline & 7,53 & PMVE $<H<P H M A$ & $0201 / 2014: 04 h$ & 0,23 & 0,43 & $02 / 01 / 2014: 20 h$ & 4.23 \\
\hline & 7.80 & $\mathrm{H}>\mathrm{PM}$ PE et PHMA & $03 / 01 / 2014: 05 h$ & 0,34 & 0,37 & $03 / 01 / 2014: 12 \mathrm{~h}$ & 0,99 \\
\hline & 7.71 & PMVE $<H<P H M A$ & $0401 / 2014: 06 h$ & 0.29 & 0,47 & $04,01 / 2014: 13 h$ & 1.27 \\
\hline & 7,24 & PMVE $<H<P H M A$ & $06 / 01 / 2014: 07 \mathrm{~h}$ & 0,42 & 0,54 & $06101 / 2014: 14 h$ & 1,83 \\
\hline & 6.81 & $H<$ PMVE et PHMA & $07 / 01 / 2014: 08 \mathrm{~h}$ & 0,37 & 0,49 & $07 / 01 / 2014: 02 h$ & 2,16 \\
\hline & 5.70 & $\mathrm{H}<\mathrm{PMVE}$ et PHMA & $26 / 01 / 2014: 11 \mathrm{~h}$ & 0,29 & 0,37 & $26 / 01 / 2014: 07 h$ & 3,47 \\
\hline & 5,74 & $H<P M V E$ et PHMA & $27 / 01 / 2014: 13 \mathrm{~h}$ & 0,05 & 0.26 & $2701 / 2014: 22 h$ & 4,00 \\
\hline & 6.39 & $H<$ PMVE et PHMA & $28 / 01 / 2014: 14 h$ & 0.26 & 0,42 & $28101 / 2014: 22 h$ & 2.94 \\
\hline & 7.82 & $\mathrm{H}>$ PMVE et PHMA & $01 / 202 / 2014: 05 h$ & 0.28 & 0,40 & $01 / 02 / 2014: 02 h$ & 4,67 \\
\hline & 7.49 & PMVE $<H<P H M A$ & $02 / 02 / 2014: 06 \mathrm{~h}$ & 0,02 & 0.19 & $02 / 02 / 2014: 02 h$ & 3,04 \\
\hline & 7,70 & $H>$ PMVE et PHMA & $03 / 02 / 2014: 06 \mathrm{~h}$ & 0,37 & 0,43 & $03 / 02 / 2014: 14 h$ & 1,69 \\
\hline & 7.25 & PMVE $<H<$ PHMA & $040212014: 19 \mathrm{~h}$ & 0.76 & 0.76 & $040212014: 19 n$ & 7,25 \\
\hline & 7,17 & PMVE $<H<$ PHMA & $05 / 02 / 2014: 0.9 h$ & 0,67 & 0.77 & $05 / 02 / 2014: 04 h$ & 3,65 \\
\hline & 6.37 & $\mathrm{H}<$ PMVE et PHMA & $D 6 / 02 / 2014=09 h$ & 0.45 & 0,86 & $06 / 02 / 2014: 20 \mathrm{~h}$ & 6.11 \\
\hline & 5,88 & $H<P M V E$ et PHMA & $07 / 02 / 2014: 22 \mathrm{~h}$ & 0,58 & 0,80 & $07 / 02 / 2014: 21 \mathrm{~h}$ & 5,73 \\
\hline & 5,69 & $H<P M N E$ et PHMA & $08 / 02 / 2014: 11 \mathrm{~h}$ & 0,56 & 0,58 & $08 / 02 / 2014: 13 h$ & 5,07 \\
\hline & 5,62 & $H<$ PMVE et PHMA & $09002 / 2014: 00 \mathrm{~h}$ & 0,45 & 0,45 & $09102 / 2014: 00 h$ & 5.62 \\
\hline & 6.22 & $H<P M V E$ et PHMA & $12002 / 2014: 12 \mathrm{~h}$ & 0,27 & 0,85 & $12 / 02 / 2014: 12 \mathrm{~h}$ & 4,62 \\
\hline & 6,42 & $H<$ PMVE et PHMA & $13 / 02 / 2014: 03 h$ & 0,24 & 0,40 & $13 / 02 / 2014: 10 h$ & 2,19 \\
\hline & 7,05 & PMVE $<H<P H M A$ & $14 / 02 / 2014: 16 \mathrm{~h}$ & 0,65 & 0,97 & $14 / 02 / 2014: 19 h$ & 5,09 \\
\hline & 6.88 & PMVE $<H<$ PHMA & $15 / 02 / 2014.04 h$ & 0,30 & 0,48 & $15 / 02 / 2014: 10 h$ & 2,04 \\
\hline & 7,14 & PMVE $<H<P H M A$ & $28 / 02 / 2014: 03 \mathrm{~h}$ & 0,21 & 0,26 & $20 / 02 / 2014: 01 h$ & 5,67 \\
\hline & 7,36 & PMVE $<H<P H M A$ & $01 / 03 / 2014: 16 \mathrm{~h}$ & 0,17 & 0.24 & $01 / 03 / 2014: 14 h$ & 5,35 \\
\hline & 7.69 & PMVE $<\mathrm{H}=\mathrm{PHMA}$ & $02 / 03 / 2014: 17 \mathrm{~h}$ & 0,40 & 0,47 & $02 / 03 / 2014: 16 h$ & 7,35 \\
\hline & 7.82 & $H>$ PMVE et PHMA & $03 / 03 / 2014: 05 h$ & 0,34 & 0,49 & $03 / 03 / 2014: 03 h$ & 5.69 \\
\hline
\end{tabular}

\begin{tabular}{|c|c|c|c|c|c|c|c|}
\hline \multirow{31}{*}{$\begin{array}{l}\text { Roscoll } \\
\text { PMNE : } 8,85 \\
\text { PHMA : } 9,76\end{array}$} & 8.06 & $H<P M V E$ et PHMA & $23 / 12 / 2013: 21 \mathrm{~h}$ & 0,52 & 0.66 & $23 / 12 / 2013: 23 n$ & 6.93 \\
\hline & 8,09 & $H<P M N E$ et PHMA & $24 / 12 / 2013: 09 \mathrm{~h}$ & 0,44 & 0.64 & $24 / 12 / 2013: 01 \mathrm{~h}$ & 4,72 \\
\hline & 7,62 & $H<$ PMVE et PHMA & $25 / 12 / 2013=10 \mathrm{~h}$ & 0,27 & 0,47 & $25 / 12 / 2013: 05 h$ & 4,24 \\
\hline & 7,45 & $H<P M V E$ et PHIMA & $27 / 12 / 2013: 12 \mathrm{~h}$ & 0,31 & 0,52 & $27 / 12 / 2013: 05 h$ & 4,19 \\
\hline & 7,55 & $H<P M N E$ et PHSMA & $28 / 12 / 2013,01 \mathrm{~h}$ & 0,37 & 0,37 & $28 / 12 / 2013: 01 \mathrm{~h}$ & 7,55 \\
\hline & 9,30 & PMVE $<H<$ PHSMA & $01 / 01 / 2014: 17 \mathrm{~h}$ & 0,31 & 0,49 & $01 / 01 / 2014: 12 \mathrm{~h}$ & 2,20 \\
\hline & 9.50 & PMVE $<H<$ PHMA & $02 / 01 / 2014=05 \mathrm{~h}$ & 0,17 & 0,39 & $02 / 01 / 2014: 23 h$ & 2,25 \\
\hline & 9,76 & PMVE $<H=$ PHMA & $03 / 01 / 2014: 06 h$ & 0,24 & 0.44 & $03 / 01 / 2014: 11 \mathrm{~h}$ & 2,75 \\
\hline & 9,70 & PMVE $<H<P H M A$ & $04 / 01 / 2014: 07 \mathrm{~h}$ & 0,19 & 0.40 & $04 / 01 / 2014: 14 h$ & 1,27 \\
\hline & 9,19 & PMVE $<H<P H M A$ & $06 / 01 / 2014: 09 h$ & 0,37 & 0,47 & $06101 / 2014: 15 h$ & 2.00 \\
\hline & 8.50 & $H<$ PMVE et PHMA & $07 / 01 / 2014: 21 \mathrm{~h}$ & 0,17 & 0.34 & $0701 / 2014: 03 h$ & 2,42 \\
\hline & 7,24 & $H<$ PMVE et PHMA & $26 / 01 / 2014: 12 \mathrm{~h}$ & 0,15 & 0,32 & $26 / 01 / 2014: 08 h$ & 4,47 \\
\hline & 7.44 & $H<$ PMVE et PHMA & $27 / 01 / 2014: 14 h$ & 0,03 & 0.21 & $27 / 01 / 2014: 22 h$ & 4,00 \\
\hline & 8.19 & $H<$ PMVE et PHMA & $28 / 01 / 2014: 15 h$ & 0.24 & 0.35 & $28 / 01 / 2014: 23 h$ & 3.64 \\
\hline & 9,88 & $H>$ PMVE et PHMA & $01 / 02 / 2014.06 \mathrm{~h}$ & 0,22 & 0,36 & $01102 / 2014: 01 \mathrm{~h}$ & 1.65 \\
\hline & 9.67 & PMVE $<H<$ PHMA & $02022014: 07 \mathrm{~h}$ & 0,00 & 0.11 & $02 / 02 / 2014: 03 h$ & 3.67 \\
\hline & 9.72 & PMVE $<H<$ PHMA & $03 / 022014: 07 h$ & 0,35 & 0.39 & $03 / 02 / 2014: 16 h$ & 3.66 \\
\hline & 9.06 & PMVE $<H<$ PHMA & $04 / 02 / 2014: 21 \mathrm{~h}$ & 0,59 & 0,60 & $0402 / 2014: 20 h$ & 9.07 \\
\hline & 9.04 & PMVE $<H<$ PHMA & $05 / 02 / 2014: 09 h$ & 0,57 & 0.74 & $05,02 / 2014: 05 h$ & 4,51 \\
\hline & 8.25 & $\mathrm{H}<\mathrm{PMVE}$ et PHMA & $06 / 02 / 2014: 10 \mathrm{~h}$ & 0,48 & 0,62 & $08 / 02 / 2014: 22 \mathrm{~h}$ & 8,11 \\
\hline & 7,56 & $H<$ PMVE et PHMA & $07 / 02 / 2014: 23 h$ & 0,54 & 0,58 & $07 / 02 / 2014: 22 \mathrm{~h}$ & 7,44 \\
\hline & 7,24 & $H<$ PMVE et PHMA & $08 / 02 / 2014: 24 \mathrm{~h}$ & 0,51 & 0,68 & $08 / 02 / 2014: 05 h$ & 4,27 \\
\hline & 6,97 & $H<P M V E$ et PHIMA & $09 / 02 / 2014: 00 \mathrm{~h}$ & 0,27 & 0,41 & $09 / 02 / 2014: 05 h$ & 4,77 \\
\hline & 8,06 & $\mathrm{H}<$ PMVE et PHMA & $12002 / 2014: 16 \mathrm{~h}$ & 0,30 & 0,76 & $12 / 02 / 2014: 13 h$ & 6,01 \\
\hline & 8.25 & $H<$ PMVE et PHMA & $13 / 02 / 2014: 04 \mathrm{~h}$ & 0,16 & 0,40 & $13 / 02 / 2014: 11 \mathrm{~h}$ & 2,63 \\
\hline & 9,05 & PMVE $<H<P H M A$ & $14 / 02 / 2014: 17 \mathrm{~h}$ & 0,73 & 0,78 & $14 / 02 / 2014: 20 h$ & 6,69 \\
\hline & 8.81 & $H<$ PMVE et PHIMA & $15 / 02 / 2014: 06 \mathrm{~h}$ & 0,31 & 0.49 & $15 / 02 / 2014: 01 \mathrm{~h}$ & 3.41 \\
\hline & 9.16 & PMVE $<H<P H M A$ & $28 / 02 / 2014: 04 h$ & 0.24 & 0,36 & $28 / 02 / 2014: 01 h$ & 5,57 \\
\hline & 9.39 & PMVE $<H<P H M A$ & $01 / 03 / 2014: 17 \mathrm{~h}$ & 0.20 & 0,22 & $01 / 03 / 2014: 16 h$ & 8,66 \\
\hline & 9.76 & PMVE $<H=P H M A$ & $0203 / 2014: 18 \mathrm{~h}$ & 0,38 & 0,45 & $02 / 03 / 2014: 17 \mathrm{~h}$ & 9,32 \\
\hline & 9.87 & $H>$ PMVE et PHMA & $03 / 03 / 2014: 06 h$ & 0,32 & 0,38 & $03 / 03 / 2014: 04 h$ & 7,19 \\
\hline
\end{tabular}

\begin{tabular}{|c|c|c|c|c|c|c|c|c|c|}
\hline \multirow{31}{*}{$\begin{array}{l}\text { Concarneau } \\
\text { PMVE : } 5,00 \\
\text { PHMA : } 5,63\end{array}$} & 4.73 & $H<$ PMVE et PHMA & $23 / 12 / 2013: 1$ & $19 \mathrm{~h}$ & 0.46 & 0.75 & $23 / 12 / 2013: 2$ & & 3,54 \\
\hline & 4.74 & H $<$ PMVE et PHMA & $24 / 12 / 2013=0$ & $08 \mathrm{~h}$ & 0.41 & 0.82 & $24 / 12 / 2013: 0$ & $01 \mathrm{~h}$ & 2,75 \\
\hline & 4.53 & $H<$ PMVE et PHMA & $25 / 12 / 2013:$ & $09 \mathrm{~h}$ & 0.36 & 0.47 & $25 / 12 / 2013: 1$ & $11 \mathrm{~h}$ & 3,97 \\
\hline & 4.49 & $H<$ PMVE et PHMA & $27 / 12 / 2013$ & $10 \mathrm{~h}$ & 0.41 & 0.49 & $27 / 12 / 2013: 0$ & $66 \mathrm{~h}$ & 2.56 \\
\hline & 433 & $H<$ PMVE et PHMA & $28 / 122014$ & $24 \mathrm{~h}$ & -0.03 & 0.22 & $28 / 12 / 2014: 0$ & agh & 3.02 \\
\hline & 5.63 & PMVE $<H=$ PHMA & $01 / 01 / 2014:$ & $16 \mathrm{~h}$ & 0.45 & 0.50 & $01 / 01 / 2014: 1$ & $13 \mathrm{~h}$ & 4.28 \\
\hline & 5.72 & $H>$ PMVE et PHMA & $0201 / 2014$ & $04 \mathrm{~h}$ & 0.22 & 0.37 & $020112014: 2$ & $20 \mathrm{~h}$ & 2.87 \\
\hline & 5.84 & $H>$ PMVE ef PHMA & $03 / 01 / 2014$ & $05 \mathrm{~h}$ & 0.28 & 0.30 & $03101 / 2014$ & $24 \mathrm{~h}$ & 1,12 \\
\hline & 5.91 & H>PMVE of PHMA & $04 / 01 / 2014:$ & $06 \mathrm{~h}$ & 0.43 & 0.44 & $0401 / 2014=0$ & $07 \mathrm{~h}$ & 5,40 \\
\hline & 5.37 & PMVE $<H<$ PHMA & $06 / 01 / 2014:$ & $07 \mathrm{~h}$ & 029 & 0.54 & $D 6 / 01 / 2014: 1$ & $13 \mathrm{~h}$ & 1,64 \\
\hline & 5.00 & PMVE $=H<$ PHMA & $07 / 01 / 2014:$ & $08 \mathrm{~h}$ & 0.23 & 0.26 & $07 / 01 / 2014$ & $13 \mathrm{~h}$ & 2,08 \\
\hline & 426 & $\mathrm{H}<$ PMVE et PHMA & $26 / 01 / 2014$ & $11 \mathrm{~h}$ & 0.17 & $\overline{0.17}$ & $26 / 01 / 2014$ & ith & 426 \\
\hline & 429 & $\mathrm{H}<$ PMVE et PHMA & $27 / 01 / 2014$ : & $13 \mathrm{~h}$ & 0,00 & 0,13 & $27 / 01 / 2014: 1$ & $19 \mathrm{~h}$ & 1,85 \\
\hline & 4.81 & $H<$ PMVE et PHMA & $2801 / 2014$ : & $14 \mathrm{~h}$ & 0.23 & 0.38 & $28 / 01 / 2014$ & $24 \mathrm{~h}$ & 4,47 \\
\hline & 5.78 & $H>P M V E$ ef PHMA & $01 / 02 / 2014:$ & $05 \mathrm{~h}$ & 0,17 & $\overline{0.22}$ & $01 / 02 / 2014$ & Dih & 3,00 \\
\hline & 5.58 & PMVE $<H<$ PHMA & $02 / 02 / 2014:$ & $05 \mathrm{~h}$ & 0,05 & 0,06 & $02 / 02 / 2014: 2$ & $23 \mathrm{~h}$ & 0,87 \\
\hline & 5,72 & $H>P M V E$ et PHMA & $03 / 02 / 2014=$ & $06 \mathrm{~h}$ & 0.25 & 0.42 & $03 / 02 / 2014=0$ & $09 \mathrm{~h}$ & 3,71 \\
\hline & 5.47 & PMVE $<H<P H M A$ & $04 / 02 / 2014:$ & $19 \mathrm{~h}$ & 0.67 & 0.70 & $04 / 02 / 2014: 2$ & $20 \mathrm{~h}$ & 5,32 \\
\hline & 5.28 & PMVE $<H<P H M A$ & $0510222014: 0$ & $07 \mathrm{~h}$ & 0.50 & 0,58 & $05 / 02 / 2014: 0$ & $04 \mathrm{~h}$ & 3,39 \\
\hline & 4.85 & $\mathrm{H} \leq \mathrm{PMV}$ E et PHMA & $0602 / 2014:$ & $09 \mathrm{~h}$ & 0.53 & 0.62 & $06 / 02 / 2014$ & $11 \mathrm{~h}$ & 4.04 \\
\hline & 4.40 & $H<$ PMVE et PHMA & $07 / 102 / 2014: 2$ & $22 \mathrm{~h}$ & 0,47 & 0.54 & $07 / 02 / 2014: 2$ & $23 \mathrm{~h}$ & 4,11 \\
\hline & 4.28 & $H<$ PMVE et PHMA & $08 / 02 / 2014: 1$ & $13 \mathrm{~h}$ & 0.45 & 0.53 & $08 / 02 / 2014: 0$ & $0 \mathrm{ho}$ & 3,84 \\
\hline & 438 & $H<$ PMVE et PHMA & $090222014: 2$ & $24 \mathrm{~h}$ & 0.42 & 0.42 & $09 / 02 / 2014: 2$ & $24 \mathrm{~h}$ & 4.38 \\
\hline & 4.67 & $\mathrm{H}<$ PMVE et PHMA & $120222014: 1$ & $13 \mathrm{~h}$ & 0.28 & 0.44 & $120272014: 1$ & $15 \mathrm{~h}$ & 4,45 \\
\hline & 4.85 & $H<$ PMVE et PHMA & $13,02 / 2014=$ & $03 \mathrm{~h}$ & 0.20 & 0.42 & $13 / 02 / 2014: D$ & $05 \mathrm{~h}$ & 4.28 \\
\hline & 5.31 & PMVE $<H<$ PHMA & $14 / 02 / 2014:$ & $15 \mathrm{~h}$ & 0,64 & 0,71 & $14 / 02 / 2014: 1$ & $17 \mathrm{~h}$ & 5,12 \\
\hline & 5.16 & PMVE $<H<$ PHMA & $15,02 / 2014: 0$ & $04 \mathrm{~h}$ & 0.20 & 0,37 & $15 / 02 / 2014=0$ & $07 \mathrm{~h}$ & 3,55 \\
\hline & 5.31 & PMVE $<H<P H M A$ & $280222014: 0$ & $03 \mathrm{~h}$ & 0.09 & 0.16 & $29101 / 2014: 0$ & $02 \mathrm{~h}$ & 5.26 \\
\hline & 5.47 & PMVE $<H<$ PHMA & $01 / 103 / 2014: 0$ & $04 \mathrm{~h}$ & 0.04 & 0.20 & $01 / 02 / 2014: 2$ & $23 \mathrm{~h}$ & 1,00 \\
\hline & 5.81 & $H>P M V E$ et PHMA & $02 / 03 / 2014: 0$ & $04 \mathrm{~h}$ & 0.21 & 0,35 & $02 / 02 / 2014: 1$ & $17 \mathrm{~h}$ & 5,71 \\
\hline & 5.75 & $\mathrm{H}>\mathrm{PMVE}$ et PHMA & $03 / 03 / 2014: 0$ & $05 \mathrm{~h}$ & 0,16 & 0.30 & $03 / 03 / 2014: 0$ & $08 \mathrm{~h}$ & 3,41 \\
\hline
\end{tabular}


Les lignes en grisé illustrent les épisodes durant lesquels la surcote maximum instantanée a coïncidé avec une pleine mer.

Shaded lines show the episodes where instantaneous maximum surge coincided with high tide level.

12Si l'on fait la synthèse de ces données hydrodynamiques complétées par des observations météorologiques (vent et pression), trois épisodes combinant une tempête et une forte marée de vive-eau d'un coefficient supérieur à 105, ont été retenus comme étant les évènements les plus morphogènes de l'hiver (tab. 4). Le premier concerne la période qui s'étend du 1 au 5 janvier 2014 durant laquelle les coefficients de marée ont atteint 108, notamment le vendredi 3 janvier. Durant cette période, la houle orientée plutôt O-SO (entre $260^{\circ}$ et $270^{\circ}$ ) a atteint des hauteurs significatives et maximum respectivement supérieures à $8 \mathrm{~m}$ et $15 \mathrm{~m}$, notamment entre les 3 et 4 janvier. Les surcotes instantanées ont été comprises entre $0,30 \mathrm{~m}$ et $0,50 \mathrm{~m}$ suivant les secteurs, mais généralement toujours plus élevées au sud de la Bretagne. Elles n'ont toutefois jamais été combinées à une pleine-mer, à l'exception du 4 janvier à Concarneau. Ces surcotes ont été générées par des vents d'afflux compris entre 18 et $20 \mathrm{~m} / \mathrm{s}$, et des pressions moyennes d'environ $996 \mathrm{hpa}$, notamment entre les 3 et 4 janvier. Cela s'est traduit par des niveaux marégraphiques toujours supérieurs aux niveaux de pleine mer de vive-eau (PMVE), et/ou égaux ou supérieurs aux niveaux des plus hautes mers astronomiques (PHMA), notamment dans le sud de la Bretagne.

Tab. 4 - Caractéristiques hydrodynamiques et météorologiques des trois épisodes tempétueux les plus morphogènes de 1'hiver 2013-2014.

Tab. 4 - Hydrodynamic and meteorological characteristics of the three most morphogeneous storm events of the winter 2013-2014.

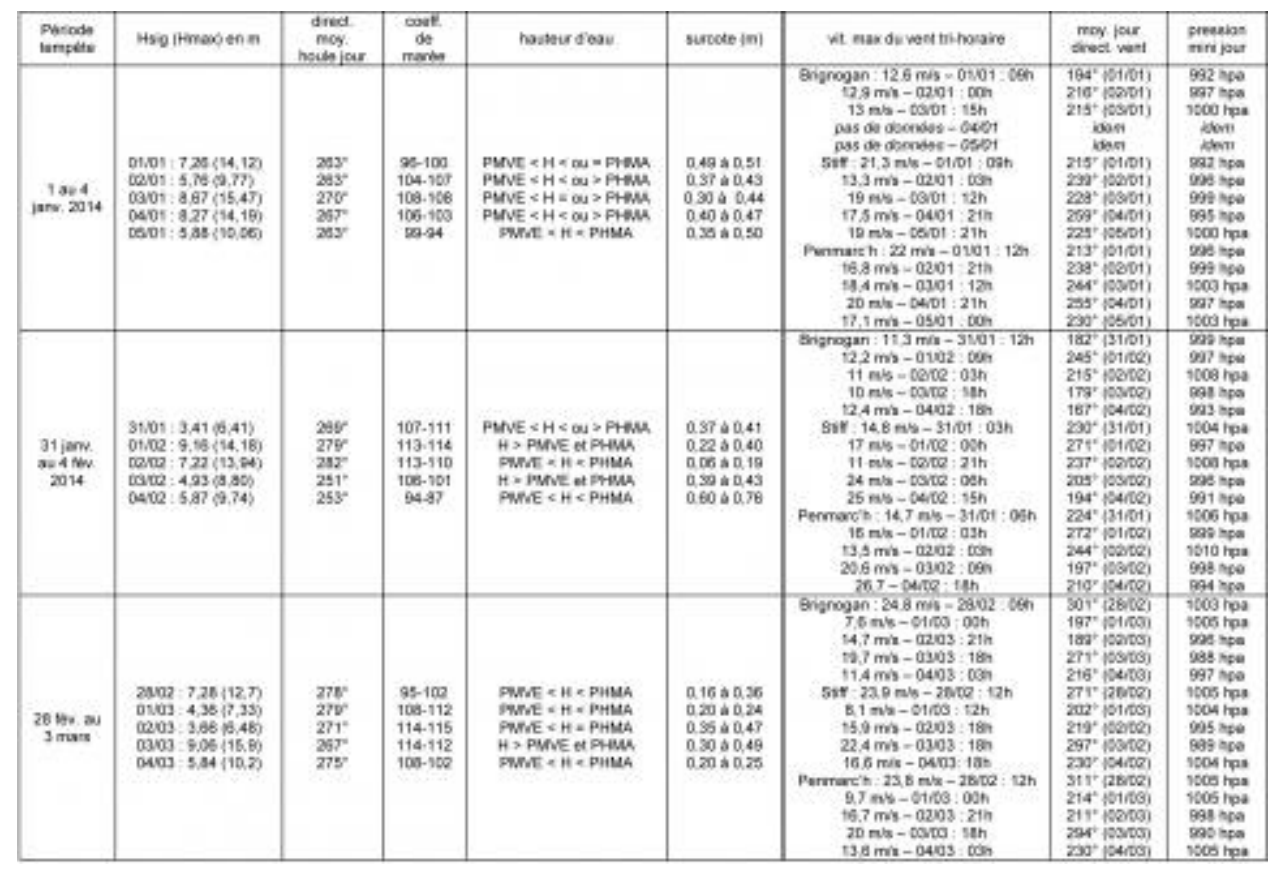

$\underline{\text { Agrandir }} \underline{\text { Original (jpeg, 1,5M) }}$

13Le second épisode est intervenu au début du mois de février, entre le 31 janvier et le 4 février 2014. Cette période a aussi été marquée par la combinaison d'une forte tempête avec 
une marée de vive-eau d'un coefficient de 113-114, notamment entre le 1 et le 2 février, alors que la houle était plutôt orientée $\mathrm{O}-\mathrm{NO}\left(269^{\circ}\right.$ à $\left.282^{\circ}\right)$. Les hauteurs significatives ont dépassé $7 \mathrm{~m}$ pour atteindre plus de $9 \mathrm{~m}$, et les hauteurs maximum plus de $14 \mathrm{~m}$, notamment le 1 février. Les surcotes instantanées ont ce jour là été comprises entre $0,20 \mathrm{~m}$ et $0,40 \mathrm{~m}$; elles ont été plus importantes en fin de période, notamment le 4 février durant la tempête Petra où elles ont atteint $0,76 \mathrm{~m}$ mais les coefficients de marées étaient alors descendants (94-87). Les observations réalisées par le Service Hydrographique et Océanographique de la Marine ont montré que pour le Conquet, les niveaux de surcote instantanés mesurés le 31 janvier étaient associés à des périodes de retour de 10 à 20 ans (Daubord, 2014). Les vents d'afflux d'ouest $\left(270^{\circ}\right.$ à $\left.271^{\circ}\right)$, de vitesses comprises entre 16 et $17 \mathrm{~m} / \mathrm{s}$, ont considérablement forci en fin de période pour atteindre plus de 25 à $26 \mathrm{~m} / \mathrm{s}$ le 4 février alors que les pressions moyennes journalières passaient de $998 \mathrm{hpa}$ à $993 \mathrm{hpa}$ (minimum $98 \mathrm{hpa}$ ). Là encore, les niveaux marégraphiques ont été en grande partie supérieurs aux PHMA, notamment le 1 février 2014.

14Le troisième et dernier épisode morphogène concerne la période du début du mois de mars qui s'étend du 28 février au 4 mars durant laquelle la tempête Christine a touché la pointe bretonne par coefficients montants de 108 à 115 entre le 1 et le 2 mars; le 3 mars, ils étaient encore de 114-112. La houle était plutôt orientée O-NO (entre $171^{\circ}$ et $179^{\circ}$ ) et les hauteurs significatives et maximum ont respectivement atteint plus de $9 \mathrm{~m}$, et presque $16 \mathrm{~m}$, notamment le 3 mars. Les surcotes maximum ont pratiquement atteint $0,5 \mathrm{~m}$, là encore le 3 mars, alors que la vitesse moyenne du vent de direction SO à $18 \mathrm{~h}$, était comprise entre $20 \mathrm{~m} / \mathrm{s}$ et $22,4 \mathrm{~m} / \mathrm{s}$ et que les pressions minimum, notamment au nord Bretagne se situaient entre $982 \mathrm{hpa}$ et $983 \mathrm{hpa}$. Les niveaux marégraphiques ont pour l'ensemble des stations d'observation été supérieurs aux PHMA cette même journée.

\subsection{Suivis morphologiques}

15Pour les cordons littoraux sableux constitués d'un cordon dunaire, les changements du trait de côte ont été suivis à partir de deux indicateurs: (i) la limite de la végétation dunaire comme étant la ligne de référence planaire du rivage, (ii) la morphologie du front de dune à partir du profil de la plage/dune le long de radiales perpendiculaires au rivage. Ces deux indicateurs sont en effet de bons marqueurs de la dynamique du trait de côte (Boak et Turner, 2005). Toutefois, suivant le contexte, il convient d'être prudent quant à leur utilisation. Dans la plupart des cas, l'érosion du trait de côte se traduit par l'apparition d'une falaise d'érosion. Dans ce contexte, la mesure planaire de la limite de végétation dunaire ou du profil de plage/dune en deux dimensions, rend très bien compte de la dynamique de recul de la ligne de rivage (fig. 5A et 5B). Néanmoins, il arrive que le couvert végétal soit simplement dégradé ou recouvert d'un saupoudrage sableux par overwash lors de la submersion, sans qu'il n'y ait pour autant d'érosion de la dune. Ce type de processus se rencontre généralement dans un contexte de plages à caractère réfléchissant à intermédiaire. Elles sont généralement caractérisées par un haut de plage très court et pentu dont le contact avec la dune de petite taille reste très flou, ce qui fait que la végétation dunaire colonise très facilement la berme du haut de plage (fig. 5C). Dans ce cas, la mesure post-tempête de la limite de végétation dunaire, comme du profil de plage/dune, ne permet pas d'identifier un probable changement du trait de côte (fig. 5C). Ces secteurs ont de ce fait été éliminés de notre analyse partant du principe que la problématique portait uniquement sur le recul du trait de côte. Pour les cordons de galets, comme le Sillon de Talbert ou les queues de comète de l'archipel de Molène, les changements du trait de côte ont été suivis à partir de la mesure planaire de la rupture sommitale du cordon, ou à partir du déplacement complet de l'ensemble de la forme à partir de profils perpendiculaires au rivage. 
Fig. 5 - Approche méthodologique suivie pour la définition d'une limite bio-morphologique du trait de côte.

Fig. 5 - Methodological approaches to the definition of shoreline indicator based on biomorphological limits.

A : plage du Vougot (Guissény)
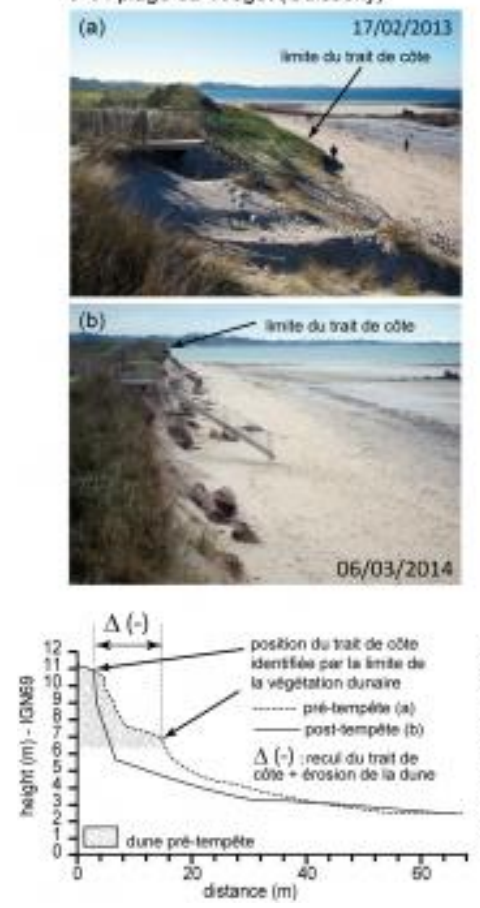
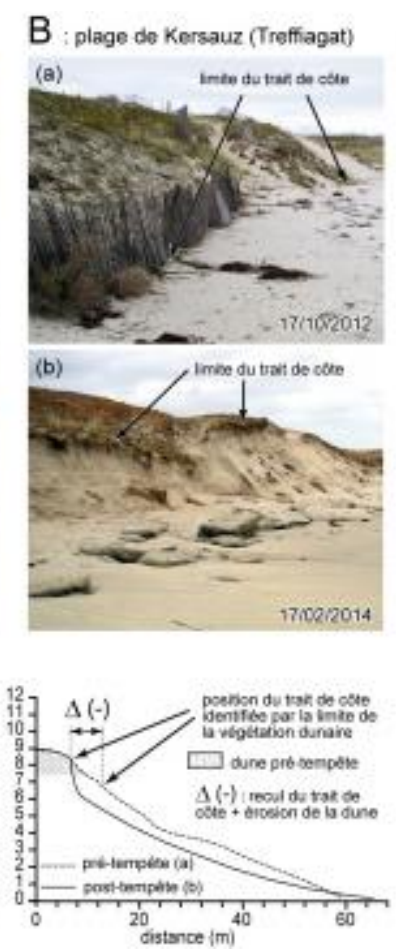
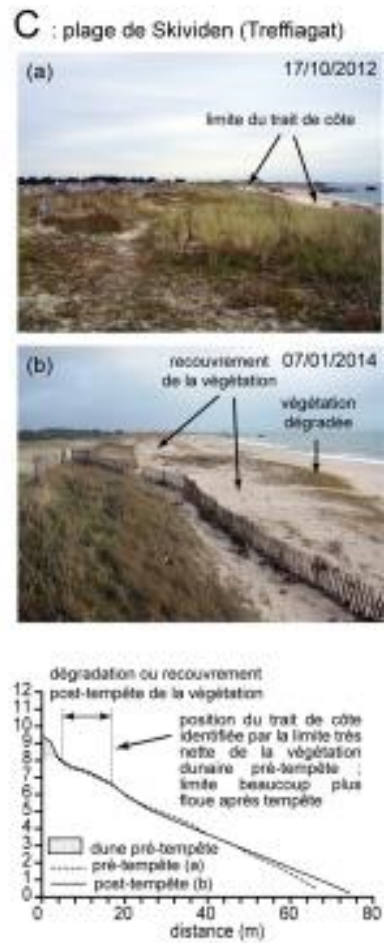

Agrandir Original (jpeg, 2,0M)

A : exemple d'un cordon dunaire dont tout le versant externe a reculé ; B : exemple d'un cordon dunaire dont seule une partie du versant externe a reculé ; $\mathrm{C}$ : exemple d'un cordon dunaire partiellement submergé sans qu'il n'y ait de recul de l'édifice. A: Example of a dune which experienced complete retreat of the seaward slope; B: Example of a dune from which only part of seaward slope has retreated;C: Example of a dune partially submerged without erosion phenomena.

16Les levés topo-morphologiques ont été effectuées au DGPS en mode RTK, ou à l'aide d'un tachéomètre laser. L'ensemble de ces mesures a été raccordé au système géodésique français à partir de bornes IGN, et au référentiel altitudinal de la France (IGN69) à partir de repères de nivellement IGN. Le tableau 5 dresse l'inventaire des levés et du type de mesures effectués en fonction des sites. Dans le cadre de cette synthèse, certains levés n'ont pas été pris en compte, notamment pour la plage de Porsmilin et du Sillon de Talbert, car ils ne montraient aucun changement morphologique significatif par rapport au relevé précédent. Cela a permis de simplifier l'analyse des données en ne retenant que les levés qui signaient le mieux les trois périodes morphogènes qui ont été inventoriées plus haut. L'état zéro a été acquis par le levé effectué juste avant la période hivernale. Lorsque cette information n'existait pas, nous avons utilisé les ortho-photographies de l'IGN réalisées durant les étés 2012 et 2013.

Tab. 5 - Inventaire des levés topomorphologiques effectués durant l'hiver 2013-2014. Tab. 5 - Inventory of topomorphological measurements achieved during the 2013-2014 winter. 


\begin{tabular}{|c|c|c|c|c|c|}
\hline \multirow{2}{*}{ sites } & \multicolumn{3}{|c|}{ Types et dates des mesures } & \multirow{2}{*}{$\begin{array}{l}\text { Laboratoires et } \\
\text { personnes }\end{array}$} & \multirow{2}{*}{$\begin{array}{c}\text { Programmes } \\
\text { de suivi }\end{array}$} \\
\hline & Profils & Trait de cote & MNT & & \\
\hline $\begin{array}{l}\text { Plage de } \\
\text { Treffiagat }\end{array}$ & $\begin{array}{l}07 / 10 / 2013 \\
07 / 01 / 2014 \\
17 / 02 / 2014 \\
04 / 04 / 2014\end{array}$ & $\begin{array}{l}07 / 10 / 2013 \\
07 / 01 / 2014 \\
04 / 04 / 2014\end{array}$ & & $\begin{array}{l}\text { Blaise E., Suanez S } \\
\text { LETG-Brest-Géomer } \\
\text { UMR } 6554 \text { CNRS }\end{array}$ & $\begin{array}{l}\text { ANR } \\
\text { COCORISCO }\end{array}$ \\
\hline $\begin{array}{l}\text { Plage de } \\
\text { Penmarch }\end{array}$ & $\begin{array}{l}07 / 10 / 2014 \\
07 / 01 / 2014 \\
17 / 02 / 2014 \\
04 / 04 / 2014\end{array}$ & $\begin{array}{l}16 / 07 / 2013 \\
08 / 01 / 2014 \\
12 / 03 / 2014\end{array}$ & & $\begin{array}{l}\text { Blaise E., Suanez S. } \\
\text { LETG-Brest-Géomer } \\
\text { UMR } 6554 \text { CNRS }\end{array}$ & $\begin{array}{l}\text { ANR } \\
\text { COCORISCO }\end{array}$ \\
\hline $\begin{array}{l}\text { Plage de } \\
\text { Porsmilin }\end{array}$ & $\begin{array}{l}04 / 12 / 2013 \\
08 / 01 / 2014 \\
30 / 01 / 2014 \\
18 / 02 / 2014 \\
03 / 03 / 2014 \\
31 / 03 / 2014\end{array}$ & $\begin{array}{l}09 / 08 / 2013 \\
08 / 01 / 2014 \\
05 / 02 / 2014 \\
18 / 02 / 2014 \\
17 / 04 / 2014\end{array}$ & & $\begin{array}{l}\text { Cuq V., David L. Suanez } \\
\text { S. LETG-Brest-Geomer } \\
\text { UMR } 6554 \text { CNRS } \\
\text { Floch F., Delacourt C. } \\
\text { LDO UMR } 6538 \text { CNRS }\end{array}$ & SNO-DYNALIT \\
\hline $\begin{array}{l}\text { lles de Trielen et } \\
\text { Lez ar Chrizienn } \\
\text { (archipel de } \\
\text { Moléne) }\end{array}$ & & $\begin{array}{l}01 / 09 / 2012 \\
20 / 01 / 2014 \\
20 / 02 / 2014 \\
17 / 04 / 2014\end{array}$ & $\begin{array}{l}\text { 30NDB au } \\
02 / 09 / 2012 \\
19-20 / 01 / 2014 \\
19-21 / 02 / 2014\end{array}$ & $\begin{array}{l}\text { Fichaut B., Suanez S. } \\
\text { Blaise E., LETG.Brest- } \\
\text { Gtomer UMR } 6554 \\
\text { CNRS } \\
\text { Davidson R., Costa S. } \\
\text { LETG-Caen-Geophen - } \\
\text { UMR } 6554 \text { CNRS }\end{array}$ & $\begin{array}{l}\text { ODC - trait de } \\
\text { cote }\end{array}$ \\
\hline $\begin{array}{l}\text { lle de Quéménez } \\
\text { (archipel de } \\
\text { Molène) }\end{array}$ & & & $\begin{array}{l}26107 / 2012 \\
15 / 05 / 2014\end{array}$ & $\begin{array}{l}\text { Stéphan P. LETG-Brest- } \\
\text { Giomer UMR } 6554 \\
\text { CNRS }\end{array}$ & $\begin{array}{l}\text { ODC - trait de } \\
\text { cote }\end{array}$ \\
\hline $\begin{array}{l}\text { Plage des Blancs } \\
\text { Sabions }\end{array}$ & & & & $\begin{array}{l}\text { Cuq V., David L. LETG- } \\
\text { Brest-Gémer UMR } 6554 \\
\text { CNRS }\end{array}$ & $\begin{array}{l}\text { ODC - trait de } \\
\text { cote }\end{array}$ \\
\hline Plage du Vougot & $\begin{array}{l}21 / 11 / 2013 \\
09 / 01 / 2014 \\
04 / 02 / 2014 \\
08 / 03 / 2014\end{array}$ & $\begin{array}{l}23 / 05 / 2013 \\
09 / 01 / 2014 \\
04 / 02 / 2014 \\
11 / 03 / 2014\end{array}$ & & $\begin{array}{l}\text { Suanez S., Blaise E. } \\
\text { LETG-Brest-Géomer } \\
\text { UMR } 6554 \text { CNRS }\end{array}$ & SNO-DYNALIT \\
\hline $\begin{array}{l}\text { Plage de } \\
\text { Boutrouilles }\end{array}$ & $\begin{array}{l}04 / 07 / 2011 \\
16 / 02 / 2014\end{array}$ & $16 / 02 / 2014$ & & $\begin{array}{l}\text { Suanez S., Blaise E. } \\
\text { LETG-Brest-Geomer } \\
\text { UMR } 6554 \text { CNRS }\end{array}$ & $\begin{array}{l}\text { ODC - trait de } \\
\text { cote }\end{array}$ \\
\hline $\begin{array}{l}\text { Baie de Saint- } \\
\text { Michel-en-Greve }\end{array}$ & & $\begin{array}{l}02 / 04 / 2013 \\
14 / 01 / 2014 \\
07 / 02 / 2014 \\
13 / 03 / 2014\end{array}$ & & $\begin{array}{l}\text { Suanez S. Stéphan P. } \\
\text { Autret R., David L. LETG- } \\
\text { Brest-Geomer UMR } 6554 \\
\text { CNRS }\end{array}$ & $\begin{array}{l}\text { ODC - trait de } \\
\text { oste }\end{array}$ \\
\hline Sillon de Talbert & $\begin{array}{l}18 / 12 / 2013 \\
07 / 01 / 2014 \\
14 / 01 / 2014 \\
28 / 01 / 2014 \\
13 / 02 / 2014 \\
20 / 02 / 2014 \\
05 / 03 / 2014 \\
\end{array}$ & & $\begin{array}{l}03-06 / 09 / 2013 \\
04-07 / 03 / 2014\end{array}$ & $\begin{array}{l}\text { Stéphan P., Fichaut B., } \\
\text { Suanez S., Blaise E, } \\
\text { Autret R., Cuq. V. LETG- } \\
\text { Brest-Gbomer UMR } 6554 \\
\text { CNRS } \\
\text { Houron. J. Maison du } \\
\text { Sillon (Pleubian) }\end{array}$ & $\begin{array}{l}\text { ODC - trait de } \\
\text { cóte }\end{array}$ \\
\hline
\end{tabular}

Agrandir Original (jpeg, 1,1M)

\section{Résultats}

\subsection{Bilan global sur le recul du rivage}

17D'un point de vue général, les résultats montrent que la dynamique de recul du trait de côte a été bien différente au cours des trois périodes morphogènes des mois de janvier, février et mars 2014 (fig. 6). Les épisodes du début du mois de janvier (du 3 et 4, et dans une moindre mesure du 6 et 7 janvier) ont généré une érosion assez limitée qui est restée en moyenne inférieure à $-2,5 \mathrm{~m}$, à l'exception de trois sites que sont la plage de Treffiagat-Léchiagat dans le sud Finistère $(-6,30 \mathrm{~m})$, de Tréduder $(-7,03 \mathrm{~m})$ et du Sillon de Talbert $(-4,65 \mathrm{~m})$ dans les Côtes d'Armor. On peut noter que pour les deux premiers secteurs les reculs maximum ont atteint respectivement $-10,63 \mathrm{~m}$ et $-16,39 \mathrm{~m}$. À l'inverse, les épisodes morphogènes du début du mois de février (celui du 1 et 2 février, suivi par la tempête Petra du 4 et 5 février) ont eu un effet érosif très important (fig. 6A). Les reculs moyens ont été dans la majorité des cas supérieurs à $-3 \mathrm{~m}$, avec des valeurs maximales supérieures ou proches de $-10 \mathrm{~m}$ comme pour la plage du Vougot $(-9,20 \mathrm{~m})$ et l'île de Lez ar Chrizienn $(-12,64 \mathrm{~m})$, respectivement à l'ouest et au nord du Finistère, ou en baie de Saint-Michel-en-Grève (entre -10,62 $\mathrm{m}$ et -17,16 m suivant les secteurs), et sur le Sillon de Talbert $(-13,4 \mathrm{~m})$ dans les Côtes d'Armor. Enfin, le troisième épisode morphogène généré par la tempête Christine du début du mois de mars a été 
le moins érosif. Les reculs moyens n'ont jamais excédé $-1 \mathrm{~m}$ à l'exception du Sillon de Talbert dans les Côtes d'Armor (-5,1 m).

Fig. 6 - Recul du trait de côte (A) généré par les trois épisodes morphogènes du début du mois de janvier, Février et mars 2014, (B) à l'échelle de l'hiver 2013-2014. Fig. 6 - Shoreline retreat (A) induced by the three morphogenetic events of early January, February and March 2014, (B) for the whole winter 2013-2014.

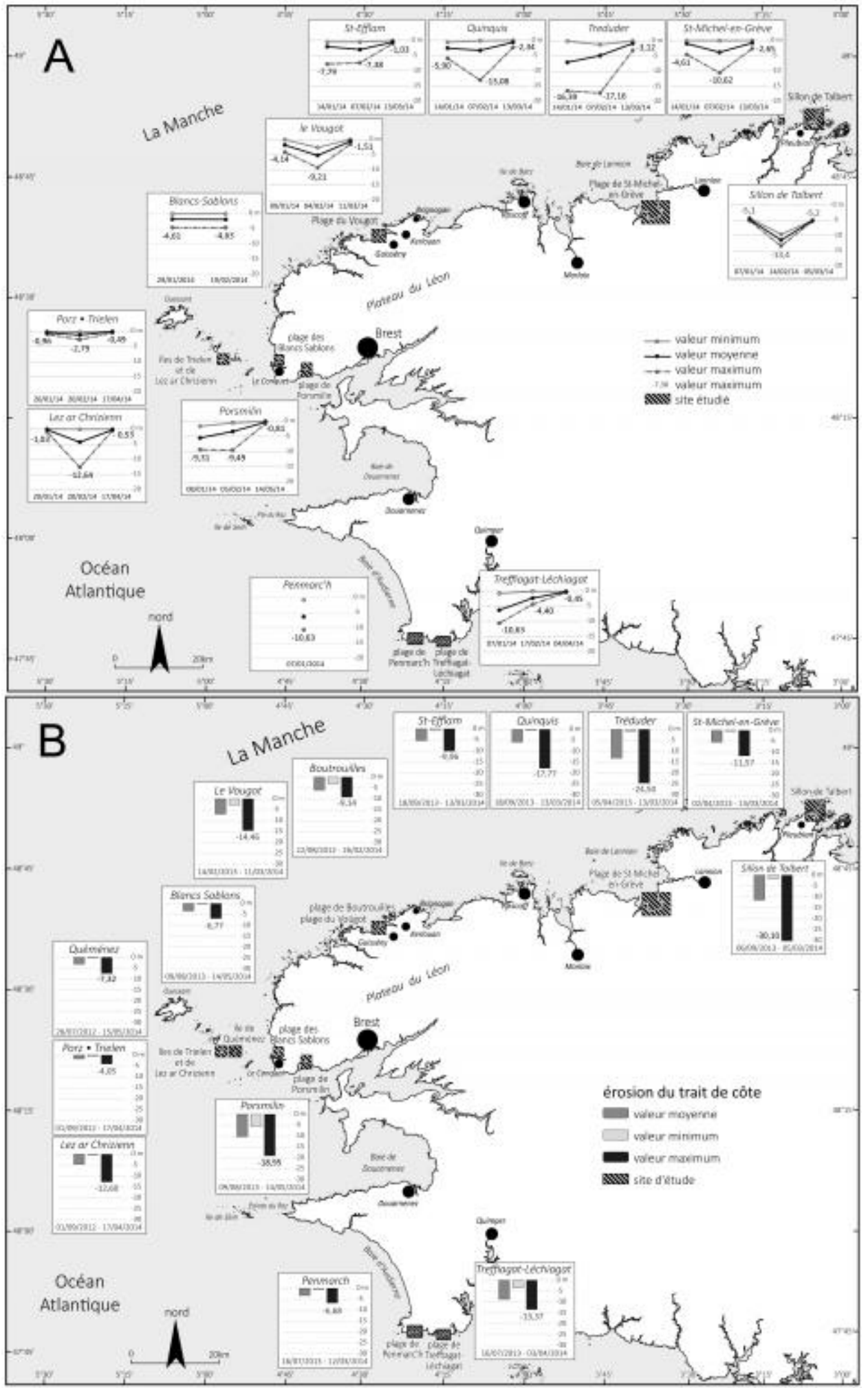


18Lorsque l'on dresse un bilan sur l'ensemble des trois périodes cumulées, le recul du trait de côte a dépassé $-10 \mathrm{~m}$ sur plus de $50 \%$ des sites étudiés, et a atteint jusqu'à $-30 \mathrm{~m}$, notamment sur le Sillon de Talbert (fig. 6B). Pour l'ensemble des secteurs, l'érosion du rivage a été supérieure ou proche de $-5 \mathrm{~m}$. Si l'on analyse ces chiffres en fonction des types de morphologie, on peut voir que, sur les îlots de l'archipel de Molène, les falaises entaillées dans des matériaux cohérents comme les plages pléistocènes ou les falaises de head et les accumulations de galets constituant notamment les queues de comètes ont beaucoup moins reculé. C'est le cas par exemple des cordons des îlots de Triélen ou de Quéménez pour lesquels les reculs maximum ont atteint respectivement $-4 \mathrm{~m}$ et $-7,3 \mathrm{~m}$. À l'inverse, les plages de sables formées d'un cordon dunaire ont enregistré des reculs bien plus importants compris entre $-6,7 \mathrm{~m}$ à Penmarc' $h$, et $-24,5 \mathrm{~m}$ à Tréduder en baie de Saint-Michel-en-Grève. Le recul maximum d'environ $-30 \mathrm{~m}$ concerne toutefois la flèche de galets à pointe libre du Sillon de Talbert.

\subsection{Erosion des dunes}

19Les plages sableuses formées d'un cordon dunaire ont été particulièrement érodées comme au Vougot sur la commune de Guissény (fig. 7). Comme le montrent les mesures effectuées le long du profil $\mathrm{n}^{\circ} 3$ situé dans le secteur de la plage qui a été le plus affecté, les trois temporalités s'expriment très clairement (fig. 7F). Le levé effectué au début du mois de janvier après la première série d'évènements morphogènes indique que le recul du trait de côte a atteint environ $-4 \mathrm{~m}$. Toutefois, les observations effectuées le 9 janvier montrent bien que le bourrelet dunaire situé au pied du versant externe de la dune est encore en place (fig. 7B). À l'inverse, les mesures effectuées le 4 février, juste après la tempête du 1-2 février, attestent de la disparition de ce dernier (fig. 7C). Le recul du trait de côte a atteint plus de $7 \mathrm{~m}$ lors de cet épisode. Les effets de la tempête Christine du 3 mars ont quant à eux été moins importants avec un recul du rivage d'environ $-4 \mathrm{~m}$ (fig. 7D et 7E). L'évolution du trait de côte de la baie de Saint-Michel-en-Grève dans les Côtes d'Armor a également enregistré un recul important suivant la même temporalité (fig. 8).

Fig. 7 - Illustration des trois phases de recul du trait de côte pour le cordon littoral sableux de la plage du Vougot (Guissény - nord Finistère).

Fig. 7 - Three phases of shoreline retreat of Vougot beach sandy coast (Guissény - North Finistère).
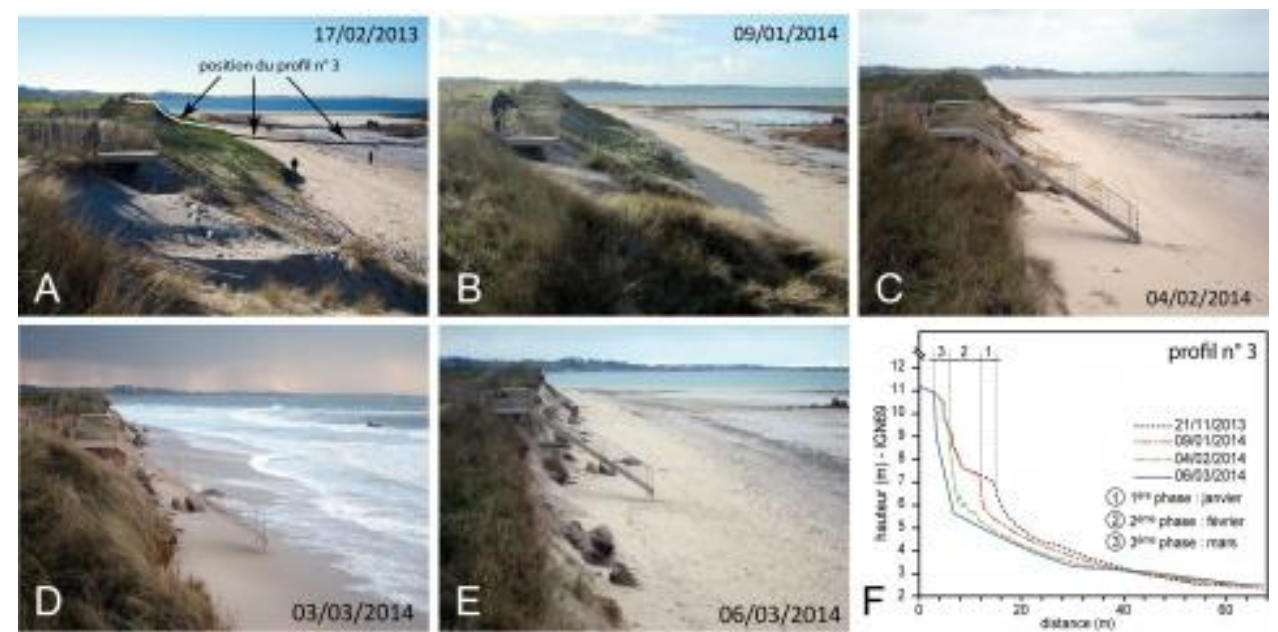
Fig. 8 - Illustration des trois phases de recul du trait de côte de la baie de Saint-Michel-enGrève (Côtes d'Armor).

Fig. 8 - Three phases of shoreline retreat for Saint-Michel-en-Grève Bay (Côtes d'Armor).

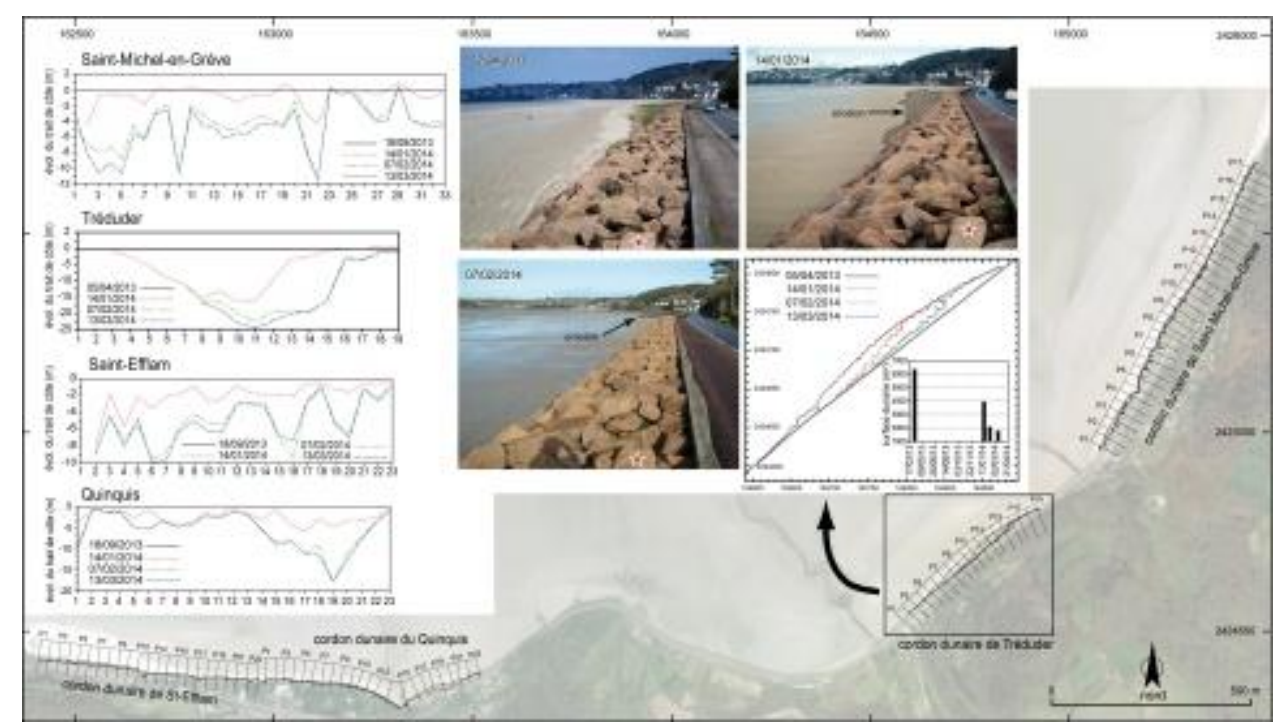

Agrandir Original (jpeg, 3,0M)

20C'est principalement l'épisode morphogène du début du mois de février qui a été le plus dommageable en matière d'érosion des dunes. Comme le montre le site de Tréduder, la surface dunaire post-tempête du début du mois de février a diminuée de $70 \%$ par rapport à avril 2013. À l'inverse, les mesures effectuées dans le sud Finistère, notamment au niveau du cordon littoral de Treffiagat-Léchiagat, montrent que l'érosion du trait de côte a été plus importante durant l'épisode morphogène du début du mois de janvier (fig. 9). Le recul de la dune de la plage de Kersauz montre clairement que dès la première phase érosive de janvier, le versant externe de la dune a connu un recul d'environ $-8 \mathrm{~m}$. Dans le même temps, son profil pentu et entièrement végétalisé a été entaillé par une falaise d'érosion d'une hauteur de plus de $2 \mathrm{~m}$. Les deux épisodes du mois de février et de mars ont été moins dommageables; durant ces deux dernières phases, le recul du front de dune a été respectivement de $-1,5 \mathrm{~m}$ et $-0,4 \mathrm{~m}$.

Fig. 9 - Illustration des trois phases de recul du trait de côte pour le cordon littoral sableux Treffiagat-Léchiagat (sud Finistère).

Fig. 9 - Three phases of shoreline retreat of Treffiagat-Léchiagat beach (South Finistère). 

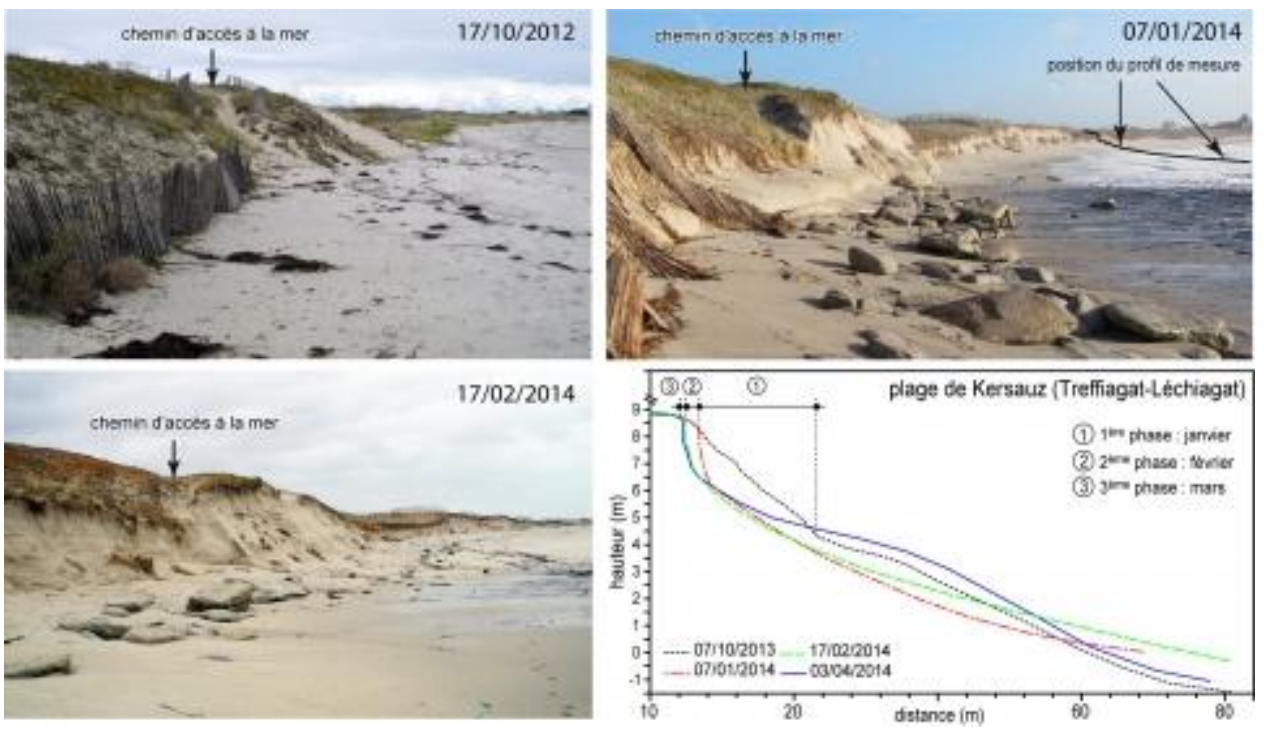

Agrandir Original (jpeg, 1,8M)

\subsection{Recul des cordons de galets}

21Pour les cordons de galets, les résultats contrastent en fonction de leur morphologie respective. Un recul du trait de côte particulièrement important a été observé sur le Sillon de Talbert. Par endroits, comme nous l'avons souligné précédemment, le recul mesuré entre les mois de septembre 2013 et de mars 2014 a atteint $-30 \mathrm{~m}$, notamment dans la partie du fulcrum la plus mobile. Cette dynamique de recul a pu être décomposée tempêtes après tempêtes grâce au suivi mené à haute fréquence tout au long de l'hiver au niveau de deux profils situés dans les parties les plus mobiles du cordon (fig. 10).

Fig. 10 - Illustration des trois phases de recul du trait de côte par rollover de la flèche de galets du Sillon de Talbert (Côtes d'Armor).

Fig. 10 - Three phases of shoreline retreat by rollover of gravel spit of Sillon de Talbert (Côtes d'Armor).
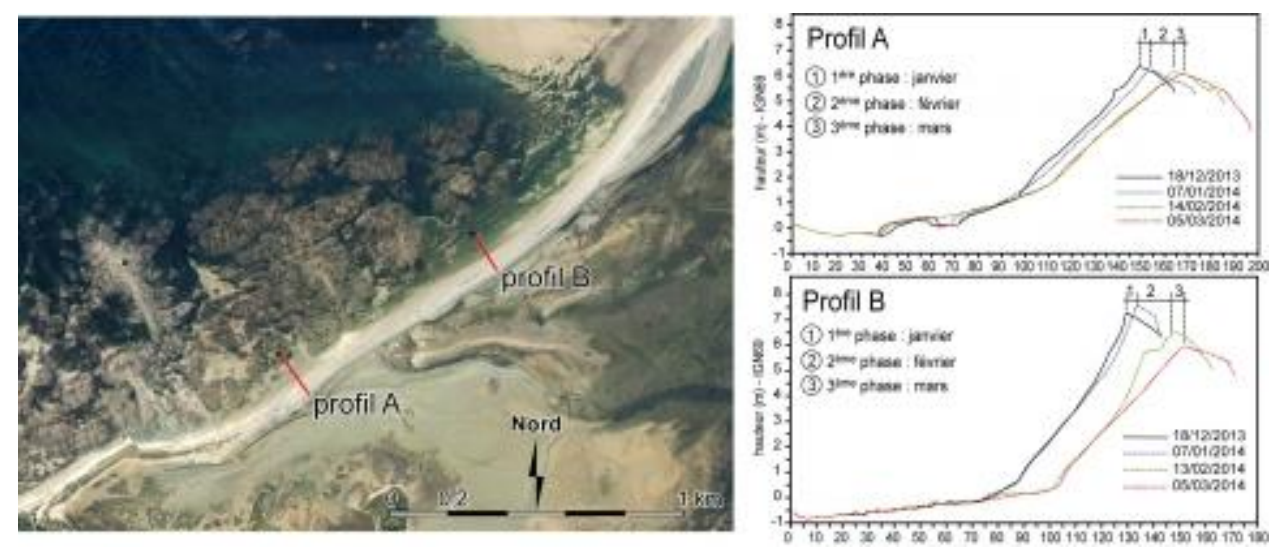

Agrandir Original (jpeg, 1,4M)

22Les données recueillies montrent que la chronologie des événements morphogènes est similaire à celle observée sur la plage du Vougot et en baie de Saint-Michel-en-Grève (fig. 10). Le recul le plus significatif est principalement intervenu durant la tempête du début 
du mois de février (entre $-10 \mathrm{~m}$ et $-15 \mathrm{~m}$ ), selon le mécanisme de rollover; ce dernier se traduit par un transfert de galets de la face avant en direction du revers sous l'effet du franchissement de la crête par les vagues de tempête (Stéphan et al., 2010). En revanche, l'épisode du début du mois de janvier a été peu morphogène, favorisant même l'exhaussement de la crête, visible au niveau du profil B. De la même façon, la tempête Christine du début du mois de mars a eu des effets limités. L'importance du recul s'explique ici par l'absence d'ados en arrière de l'accumulation et par la forte exposition du cordon aux houles incidentes. Dans le cas des accumulations de galets formant les queues de comète et des falaises entaillées dans des matériaux cohérents comme les plages pléistocènes ou les falaises de head de l'archipel de Molène, le recul du trait de côte a été beaucoup moins important. Les mesures effectuées sur les îlots de Triélen, de Lez ar Chrizienn, ou encore de Quéménez montrent que les reculs n'ont jamais excédé -7 à $-8 \mathrm{~m}$ au maximum. Seul un secteur bien particulier du cordon de Lez ar Chrizienn, dont le fonctionnement s'apparente à celui d'un musoir de flèche à pointe libre, a connu un recul plus important. Là encore, la dynamique du trait de côte montre la même temporalité quels que soient les cordons de galets étudiés dans ce secteur. À l'image de la plage de Porz située au nord du cordon de Triélen, la tempête du début du mois de janvier a eu peu d'effet sur la mobilité de la ligne de rivage (fig. 11). Sur la photo du 21 janvier, la ruine qui se situe en bordure du trait de côte est encore en place, comme elle l'était en 2012. En revanche, après l'épisode morphogène du début du mois de février, les murets ont disparu en majeure partie suite au recul important du trait de côte qui a atteint environ $-3 \mathrm{~m}$ dans ce secteur. Enfin, la tempête Christine du début du mois de mars n'a induit que de faibles variations de la ligne de rivage comme l'attestent le levé topographique ainsi que la photographie prise sur le terrain le 17 avril 2014.

Fig. 11 - Illustration des trois phases de recul du trait de côte du cordon de galets de la plage de Porz sur l'île de Triélen - archipel de Molène (ouest Finistère).

Fig. 11 - Three phases of shoreline retreat by rollover shingle beach of Porz on Triélen Island (Western Finistère). 

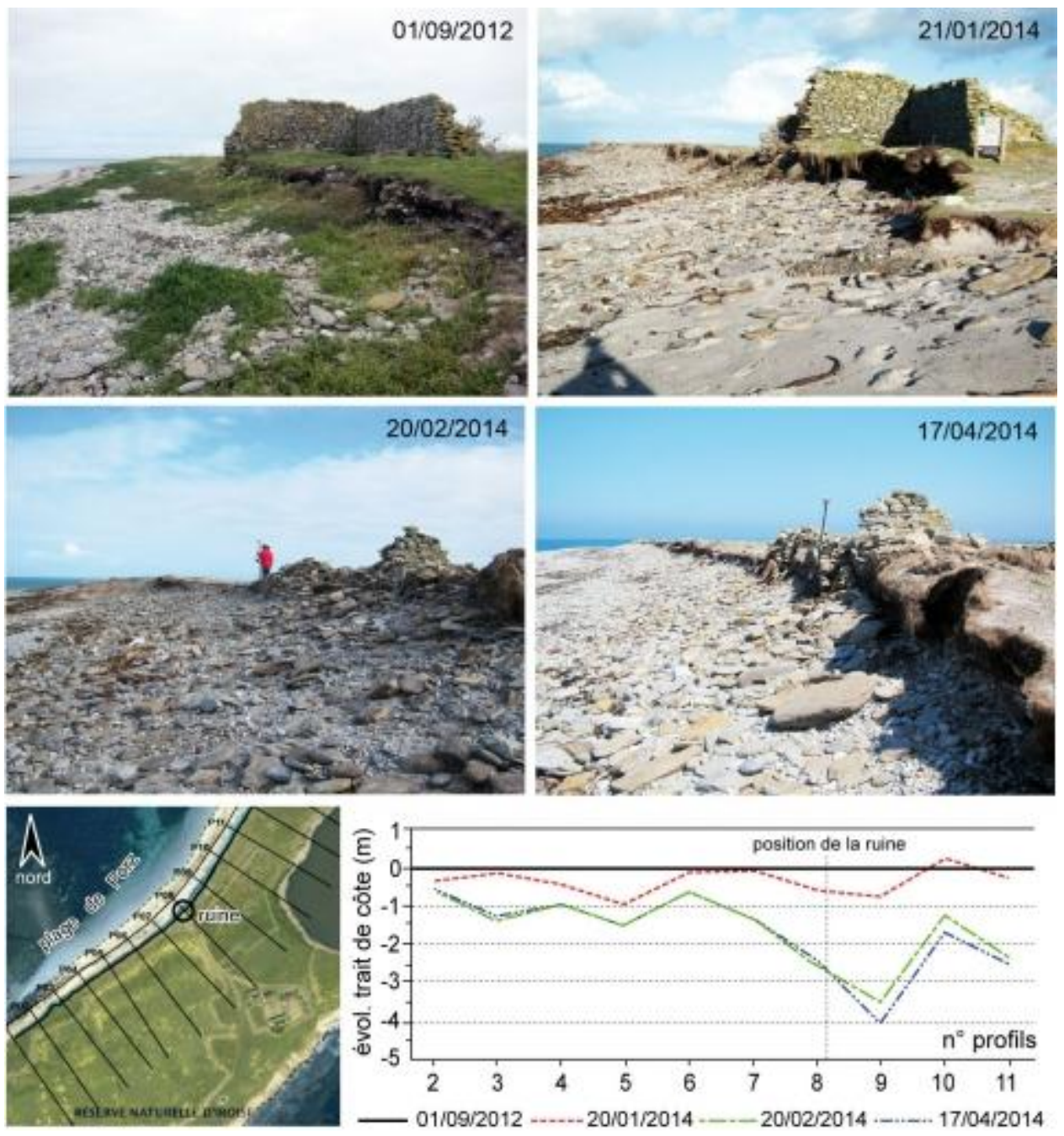

$\underline{\text { Agrandir }} \underline{\text { Original (jpeg, 548k) }}$

\subsection{Impact sur les cordons littoraux anthropisés et/ou protégés}

23Les cordons littoraux anthropisés et/ou protégés ont également été largement affectés par cette série d'épisodes morphogènes. L'érosion du trait de côte sur les plages de Boutrouilles à Kerlouan, et de Léhan à Treffiagat-Léchiagat, respectivement dans le nord et le sud Finistère, a entrainé une destruction complète des ouvrages de protection correspondant dans ces deux cas à des structures monolithiques de type STABIPLAGE ${ }^{\circledR}$ (fig. 12). Toutefois, on observe que l'épisode morphogène du début du mois de janvier a eu des effets différents sur les deux sites. Comme l'indiquent les photos diachroniques de la plage de Boutrouilles, la tempête de début janvier a eu un impact érosif très limité. La situation du 4 janvier montre que le STABIPLAGE ${ }^{\circledR}$ est toujours en place, et le versant externe du cordon dunaire est entièrement végétalisé. Seul le pied de dune à été érodé, attestant d'une submersion assez limitée de l'ouvrage de protection (fig. 12A). La destruction complète de ce dernier a eu lieu lors de l'épisode morphogène du début du mois de février comme le montre la situation du 16 février. À l'inverse, le STABIPLAGE ${ }^{\circledR}$ installé en pied de dune sur la plage de Léhan a été totalement détruit dès la tempête du début du mois de janvier (fig. 12B).

Fig. 12 - Erosion du trait de côte et destruction des ouvrages de défense du littoral de la plage Boutrouilles à Kerlouan - nord Finistère (A), et de la plage de Léhan à Treffiagat-Léchiagat - 
sud Finistère (B).

Fig. 12 - Shoreline erosion and destruction of coastal protection structures on Boutrouilles beach at Kerlouan - North Finistère (A), and Léhan beach at Treffiagat-Léchiagat - South Finistère (B).
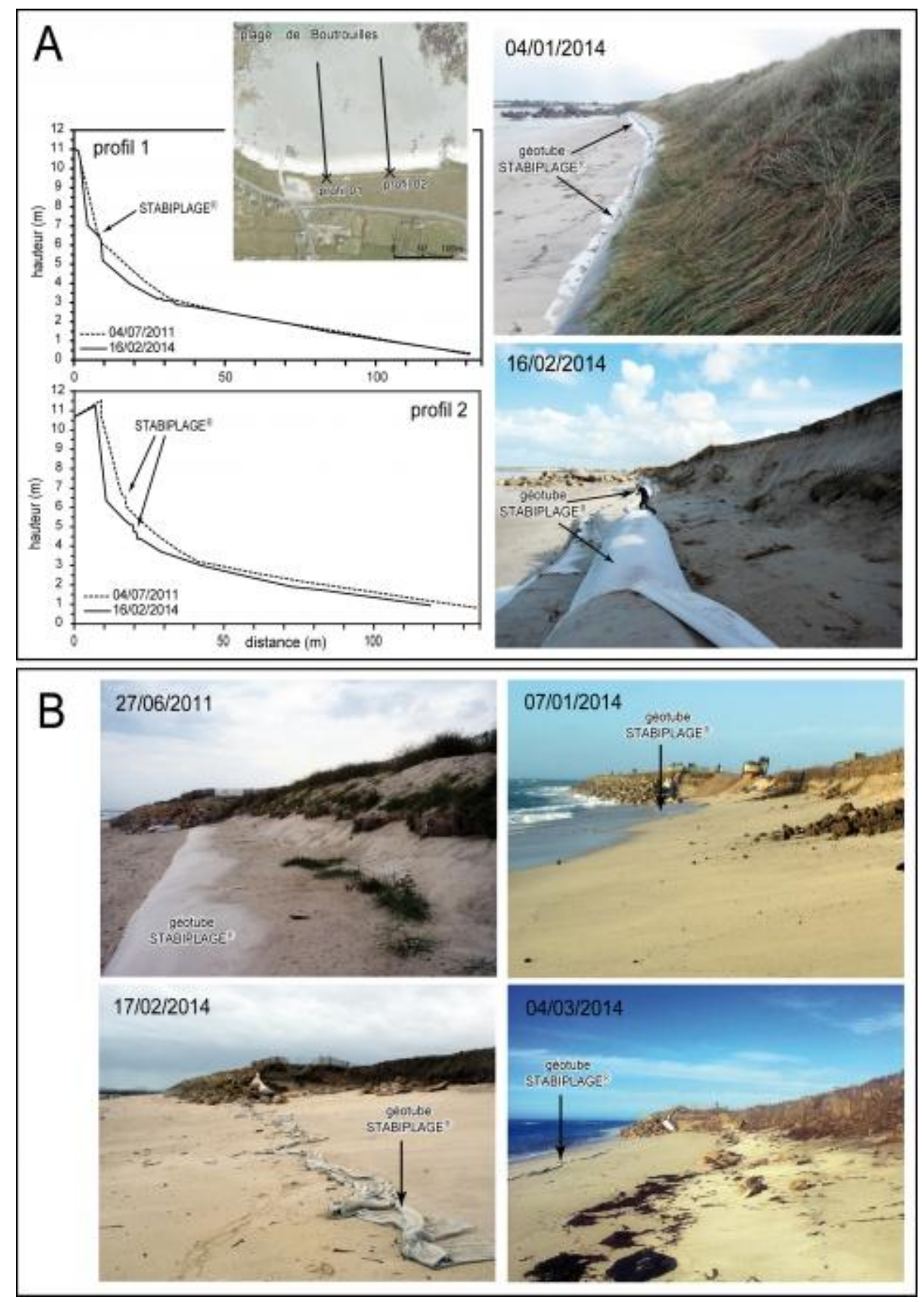

Agrandir Original (jpeg, 3,1M)

24Les mesures effectuées le 7 janvier dans ce secteur ont montré que le rivage avait reculé d'environ $-7 \mathrm{~m}$. Les épisodes morphogènes de février et mars n'ont fait qu'aggraver cette situation comme l'indiquent les levés du 3 avril attestant d'un recul d'environ $-10 \mathrm{~m}$, et ce malgré les rechargements réalisés par la municipalité après chaque tempête. Les secteurs aménagés ont également été fortement affectés comme le montre la plage de Porsmilin. À cet 
endroit, l'ancien cordon correspondant à une flèche de mi-baie barrant une ria située en arrière a été au cours du temps totalement remblayé. Il forme aujourd'hui un talus artificiel dunifié sur lequel un chemin carrossable a été aménagé de manière à desservir un parking (fig. 13). Dans le même temps, le cours d'eau s'écoulant de l'amont a été busé pour permettre l'évacuation des eaux sous le remblai. L'ensemble de ces aménagements anthropiques a été totalement détruit dès la tempête du début du mois de janvier. Comme l'indiquent les mesures effectuées le 8 janvier, le recul du trait de côte a atteint entre -4 et $-9 \mathrm{~m}$ suivant les secteurs (fig. 13A). Il en a été de même durant l'épisode du début du mois de février; les levés du 5 février montrent que l'érosion maximum se situe entre -8 et $-18 \mathrm{~m}$, notamment au niveau du parking. C'est aussi durant cet épisode morphogène que le chemin carrossable et la cale d'accès à la plage ont été totalement détruits (fig. 13B et 13D). Pour finir, on peut noter que là encore la tempête du 3 mars a été peu dommageable en matière d'érosion.

Fig. 13 - Erosion du trait de côte et destruction des aménagements littoraux de la plage de Porsmilin - ouest Finistère.

Fig. 13 - Shoreline erosion and destruction of coastal development on Porsmilin beach Western Finistère.
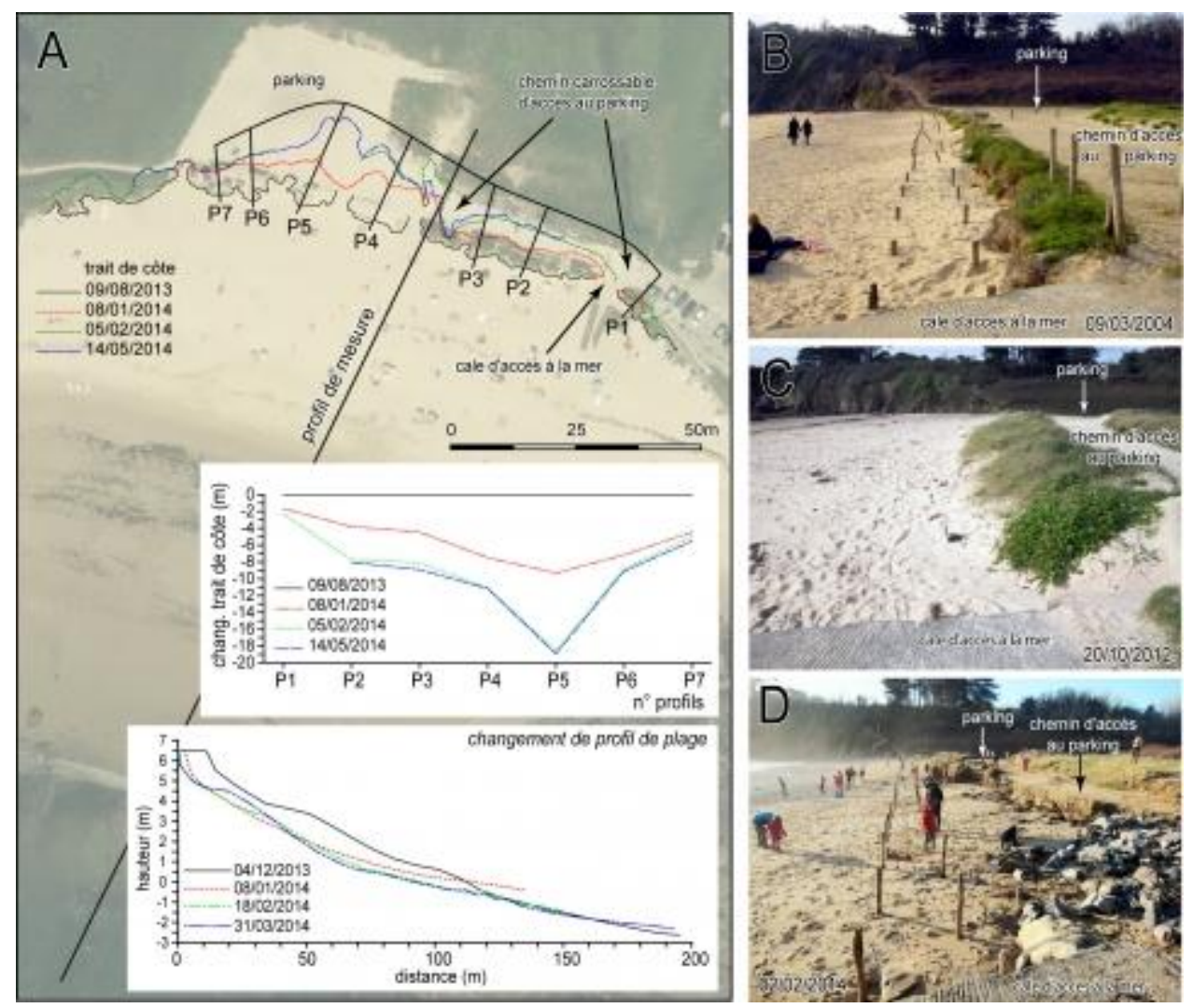

Agrandir Original (jpeg, 2,7M)

A : variation du trait de côte et changement de profils de plage; B : situation avant les tempêtes le 09/03/2004; $\mathrm{C}$ : et le 29/10/2012; D : situation après tempête le 02/02/2014. A: Shoreline and beach profile changes; B: situation before storm events 09/03/2004; C: and 29/10/2012; D: situation after storm events 02/02/2014. 


\section{Discussion}

25Les tempêtes de l'hiver 2013-2014 ont été caractérisées dans la majorité des cas comme des évènements cycloniques très rapides, dont la trajectoire très longue débute à l'ouest-sud-ouest à $60^{\circ}$ de longitude $\left(40^{\circ}\right.$ à $47^{\circ}$ de latitude) et se termine à l'est-nord-est au niveau du Danemark, entre $50^{\circ}$ et $58^{\circ}$ de latitude. Ces dépressions sont le plus souvent accompagnées de vents très violents supérieurs à 11 Beaufort (Roche et al., 2014). Elles sont qualifiées de dépressions météorologiques à cyclogénèse intense, ou «bombe » (Betts et al., 2004). Leur trajectoire zonale, plutôt décalée au nord de la Bretagne, au niveau des îles anglosaxones, est commandée par la position d'un puissant jet d'ouest à $500 \mathrm{hPa}$ circulant à $45^{\circ}$ de latitude. Toutefois, c'est surtout la répétition de ces épisodes tempétueux qui a fortement marqué la période hivernale écoulée. Il faut remonter à l'hiver 1989-1990 pour connaître une telle fréquence d'évènements tempétueux (McCallum et Norris, 1990 ; Betts et al., 2004 ; Caspar et al., 2007 ; Roche et al., 2014). En effet, entre la mi-décembre 1989 et la fin février 1990, pas moins de 15 tempêtes majeures avaient touché la pointe bretonne ; le mois de février avait été le plus tempétueux (McCallum et Norris, 1990). Les enregistrements de houle effectués à l'époque au large de l'île de Ouessant par la bouée-phare BEAIII ont montré que durant certains épisodes, comme la tempête Daria du 25 janvier (encore appelée Burn's Day en Angleterre), ou du 11 février, les hauteurs significatives de houle ont été respectivement égales à $12,2 \mathrm{~m}$ et $12,4 \mathrm{~m}$, et la hauteur maximum à $18 \mathrm{~m}$ et 18,4 $\mathrm{m}$ (Suanez et al., 2009). À titre de comparaison, les hauteurs significatives restent très proches de ce que l'on a connu cet hiver 2013-2014, notamment pour les deux tempêtes Petra du 5 février $\left(\mathrm{H}_{\mathrm{sig}}=12,3 \mathrm{~m}\right)$, et Ulla du 14 février $\left(\mathrm{H}_{\mathrm{sig}}=12,4 \mathrm{~m}\right)$. À l'inverse, la hauteur maximum reste toutefois inférieure à celles enregistrées durant l'hiver 2013-2014, notamment durant la tempête Petra où elle a atteint 23,5 m le 5 février. Cela pourrait s'expliquer par le poids considérable de la bouéephare BEAIII (80 tonnes) à l'origine d'une sous-estimation systématiquement des hauteurs des plus fortes houles (Benoit et al., 2006). Pour autant, la hauteur maximum enregistrée durant la tempête Petra reste inférieure à celles observées plus au nord du bassin nord Atlantique, à savoir 24,3 m et 29,1 m respectivement aux Shetland (Hall et al., 2006), et au large de l'Ecosse (Holliday et al., 2006). L'impact des tempêtes de l'hiver 1989-1990 sur la dynamique du trait de côte avait été tout aussi dommageable que cet hiver 2013-2014. Dans le sud Finistère, la tempête du 16-17 décembre 1989 avait ouvert de nombreuses brèches dans les cordons de Treffiagat-Léchiagat, Lesconil et de Combrit, jusqu'à $250 \mathrm{~m}$ de largeur au niveau de la plage de Kersauz (Garnier, 1993 ; Bodéré et al., 1993 ; Hallégouët et Hénaff, 2006). Des observations similaires avaient été faites en baie d'Audierne après les tempêtes de la fin décembre 1989, notamment dans les secteurs de Trunvel, et plus au sud au niveau du Loc'h ar Stang, où des brèches de plusieurs dizaines de mètres de largeur avait été ouvertes dans le cordon littoral (Hallégouët et Bodéré, 1993 ; Hallégouët et Hénaff, 1993 ; Faye et al., 2007). Dans le nord Finistère, des reculs du trait de côte avaient également été notés suite aux tempêtes de décembre 1989, notamment dans la partie orientale de la plage du Vougot à Guissény sans qu'aucune valeur fiable ne soit donnée (Garaboeuf, 1990). Toutefois, ce recul avait été suffisamment important pour que la mairie décide de mettre en place un enrochement dans ce secteur. À l'ouest du Finistère, les études réalisées sur la dynamique des blocs cyclopéens de l'île de Banneg dans l'archipel de Molène avaient aussi montré l'importance des évènements tempétueux de l'hiver 1989-1990 (Fichaut et Hallégouët, 1989). Plusieurs centaines de tonnes de dalles avaient été arrachées aux falaises ou aux amas préexistants et projetées à l'intérieur de l'île. Dans le même temps, la partie centrale de l'île culminant entre 8 et $9 \mathrm{~m}$ NGF avait été entièrement submergée par des niveaux d'eau extrêmes. Il est aussi intéressant de comparer l'ampleur de l'érosion du trait de côte mesurée cet hiver avec les reculs obtenus lors de la tempête Johanna du 10 mars 2008 qui constitue le 
dernier épisode véritablement morphogène ayant affecté la Bretagne. Cet évènement tempétueux est également survenu lors d'une pleine mer de vive-eau (coefficient 106), alors que les hauteurs significatives et maximum des houles enregistrées aux Pierres Noires atteignaient respectivement $11,5 \mathrm{~m}$ et plus de $18 \mathrm{~m}$ à $15 \mathrm{~h}$ (Cariolet et al., 2010). Cette comparaison est d'autant plus aisée que de nombreux sites présents dans cette étude faisaient déjà l'objet d'un suivi topo-morphologique à l'époque ; gardons toutefois à l'esprit que nous comparons ici l'impact érosif d'un seul événement avec une série de tempêtes. Ainsi, dans les Côtes d'Armor, le Sillon de Talbert avait reculé d'environ -22 m au maximum contre plus de $30 \mathrm{~m}$ cet hiver (Stéphan et al., 2010). En baie de Saint-Michel-en-Grève, le recul maximum des dunes avait atteint $-18 \mathrm{~m}$, notamment dans le secteur de Saint-Michel-en-Grève, contre 24,5 $\mathrm{m}$ cet hiver (Suanez et Stéphan, 2011). Sur la côte nord du Finistère, au niveau de la plage du Vougot, le recul maximum du cordon dunaire avait été d'environ $-6 \mathrm{~m}$ contre $-14 \mathrm{~m}$ cet hiver (Suanez et Cariolet, 2010). De même, le STABIPLAGE ${ }^{\circledR}$ installé en pied de dune sur la plage de Boutrouilles à Kerlouan avait été submergé sans être détruit, induisant un recul compris entre -2 et $-3 \mathrm{~m}$ de la partie basale du cordon (Suanez et Cariolet, 2010). Les tempêtes de cet hiver ont totalement détruit cet ouvrage de protection tout en générant un recul de l'ensemble de la dune d'environ $-7 \mathrm{~m}$. À l'ouest du Finistère, l'impact morphosédimentaire de la tempête Johanna dans l'archipel de Molène s'était aussi traduit par des phénomènes érosifs importants (Suanez et al., 2011). La plage de Porz située au nord de l'îlot de Triélen avait reculé de $-2,5 \mathrm{~m}$ contre $-4 \mathrm{~m}$ cet hiver. Au niveau de Lez ar Chrizienn, le recul du trait de côte avait atteint environ $-17 \mathrm{~m}$ contre $-12,5 \mathrm{~m}$ cet hiver, toujours dans la partie la plus mobile du nord de l'île. De même, les observations faites sur l'île de Banneg avaient montré l'importance des phénomènes d'arrachement et de déplacement de blocs cyclopéens (Fichaut et Suanez, 2010, 2011). Le plus gros bloc déplacé durant la tempête Johanna pesait 42 tonnes. Arraché de la roche mère à 3 mètres au-dessus des plus hautes mers de vive-eau, il s'était déposé à plus de $7 \mathrm{~m}$ de son point de départ à l'intérieur de l'île. D'une façon générale, on peut noter que le recul du trait de côte durant l'hiver 2013-2014 a été bien supérieur à ce que l'on a connu durant la tempête du 10 mars 2008, ce qui reviendrait à dire qu'une série de tempête produit bien plus de dommages qu'un événement isolé. Toutefois, comme l'ont montré nos suivis topo-morphologiques (fig. 6A), ce recul est principalement lié à l'événement du début du mois de février, ceux des mois de janvier et de mars ayant eu des effets limités sur le recul du rivage, notamment dans le nord Bretagne au mois de janvier. Il serait donc plus cohérent de comparer l'impact de la tempête du 10 mars 2008 à celle du début du mois de février 2014. Pour autant, les changements morphologiques générés par les premières tempêtes de l'hiver ont certainement joué dans l'augmentation de l'érosion durant l'épisode du mois de février, en abaissant le profil du haut de plage et en créant ainsi les conditions propices aux phénomènes de submersion et d'attaque du pied de dune par la houle. Comme l'ont souligné plusieurs auteurs, ces dynamiques illustrent les processus rétroactifs liés à l'action répétée d'une série de tempête sur les changements morphologiques du profil transversal initial de la plage (Coco et al., 2014 ; Caspar et al., 2010 ; Letortu et al., 2012 ; Sénéchal et al., 2015). Sur les plages sableuses, l'effet des tempêtes se traduit le plus souvent par une rotation du profil transversal de la plage qui accentue alors l'action érosive du runup lors des événements suivants, même lorsqu'il s'agit de tempêtes de moindre intensité (Ruz et al., 2009 ; Sénéchal et al., 2011 ; Vousdoukas et al., 2012). Néanmoins, il faut noter que le dernier épisode morphogène du mois de mars n'a pratiquement rien produit en matière d'érosion du trait de côte, et ce malgré des conditions énergétiques favorables et des coefficients de marée particulièrement forts (112 à 115). Le faible impact morphogène de ce dernier épisode hivernal s'explique par le recul important de l'ensemble des systèmes plages/dunes et des cordons de galets d'environ $-5 \mathrm{~m}$ en moyenne ( $\geq-10 \mathrm{~m}$ au maximum) sur l'ensemble des sites après la tempête du début du mois de février. Ce recul a abouti à un 
élargissement de la zone de haut de plage où interviennent le déferlement des vagues et les processus de runup participant à l'érosion du trait de côte. Ainsi, pour des niveaux d'eau extrêmes tout aussi importants, l'érosion n'agit plus (ou très faiblement) car le pied de dune n'est plus véritablement atteint. De même, ces changements morphologiques interviennent également dans le déséquilibre énergétique des vagues au déferlement, notamment des ondes infragravitaires, qui participent à l'érosion du trait de côte (Yates et al., 2009 ; Castelle et al., 2014 ; Coco et al., 2014). Ces éléments montrent que sur une longue période marquée par une série de tempête, au-delà d'un certain seuil dans le processus de recul du rivage, l'action érosive des épisodes morphogènes n'agit plus de façon significative (voir plus du tout), quelle que soit leur intensité. Comme l'ont montré Coco et al. (2014), l'effet cumulé des tempêtes sur la morphologie des plages, notamment en matière d'érosion du rivage, n'est donc pas attesté au-delà d'un certain seuil. Sur les cordons de galets, les évolutions mesurées confirment quant à elles les schémas de fonctionnement morphodynamique régionaux d'ores et déjà établis pour ce type d'accumulation sédimentaire (Stéphan et al., 2010 ; Stéphan, 2011a, 2011b). Les premiers épisodes de tempête de l'hiver constituent en quelque sorte les événements «préparateurs ». Les fortes houles associées à des coefficients de marée élevés favorisent le redressement des cordons qui s'accompagne d'un vannage de la fraction sableuse matricielle dans la partie haute et la formation d'une coiffe de galets en openwork. C'est ensuite un événement " déclencheur » qui engendre un recul important des accumulations, en l'occurrence l'épisode morphogène du début du mois de février dans le cas qui nous occupe. Toute la partie sommitale se déverse brutalement sur le revers. La partie haute du cordon devient alors moins facilement mobilisable lors des événements suivants, en raison de la nature plus compacte du cordon, composé désormais d'un mélange de sable et de galets. Cela explique en grande partie le recul limité des cordons de galets lors du dernier épisode morphogène du mois de mars, pourtant très énergétique. Si les processus de rétroaction et d'auto-organisation propres aux cordons de galets soumis à une série de tempêtes ont jusqu'à présent été décrits de façon plutôt théorique (Orford et al., 2003 ; Orford et Jennings, 2007 ; Orford et Anthony, 2011 ; Orford, 2011), ils sont ici pleinement démontrés par de la mesure in situ. Ces données soulignent une nouvelle fois le caractère non-linéaire et les effets de seuils qui caractérisent les phénomènes morphogéniques responsables de la mobilité des cordons de galets lors des tempêtes (Stéphan, 2008).

26Les singularités en matière d'impacts morphosédimentaires des trois épisodes morphogènes des mois de janvier, février et mars, tiennent également à la dynamique atmosphérique, et à travers elle, à l'orientation de la houle. Cette dernière a joué un rôle important dans la différence de réponse des cordons littoraux du sud et du nord Bretagne à certains épisodes morphogènes. Ainsi, celui du début du mois de janvier marqué par la tempête du 3-4 janvier a affecté bien plus sévèrement le sud Bretagne, et notamment le sud Finistère, car les houles avaient une incidence sud-ouest. À l'inverse, le recul du rivage a été plus important sur la côte nord de la Bretagne lors de l'épisode du début du mois de février car les houles étaient plutôt orientées O-NO. Ces mécanismes avaient aussi agi à l'échelle de la journée lors de la tempête Johanna, avec le déplacement du front froid entre le matin et le soir (Cariolet et al., 2010).

27Pour finir, il convient de discuter les raisons de la forte érosion généré par l'épisode morphogène du début du mois de février 2014, comme l'avait été également la tempête Johanna du 10 mars 2008. Comme le montrent les suivis effectués sur la plage du Vougot à Guissény, si l'on replace ces deux évènements au sein d'une évolution à plus long terme de la dynamique du trait de côte, on peut voir que ces épisodes morphogènes sont survenus alors que le système était fragilisé (fig. 14). 
Fig. 14 - Evolution du bilan sédimentaire du cordon dunaire de la plage du Vougot (Guissény) dans sa partie orientale entre les mois de juin 2004 et septembre 2014.

Fig. 14 - Sediment budget evolution of the dune of the eastern part of Vougot beach (Guissény) between June 2004 and October 2014.
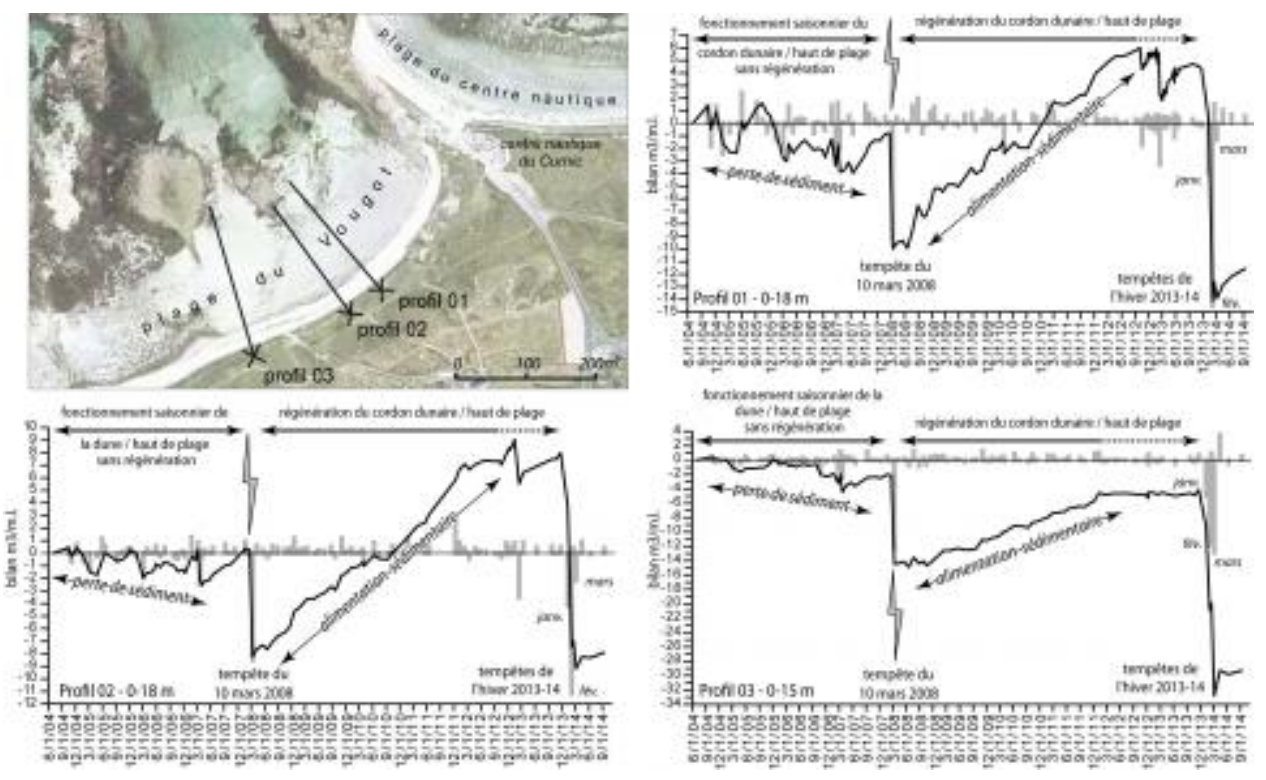

Agrandir Original (jpeg, 1,5M)

28En effet, les données acquises pour les trois profils de mesures indiquent clairement que l'érosion, notamment durant l'hiver 2006-2007, a "préparé le terrain » pour la tempête du 10 mars 2008. La perte de sédiment enregistrée durant cette période, n'ayant pas été par la suite récupérée, s'est traduite par un bilan sédimentaire déficitaire au mois de février 2008 en comparaison à la situation initiale de 2004. L'absence de régénération du système dune/haut de plage a donc joué un rôle essentiel dans l'exacerbation de l'érosion générée par la tempête Johanna, confirmant ainsi le fait que la réponse morphosédimentaire des plages aux évènements extrêmes est surtout dépendante de leur fréquence par rapport aux périodes de régénération qui les séparent (Morton et al., 1995 ; Ferreira, 2006 ; Anthony, 2013 ; Castelle et al., 2014). À l'inverse, comme ceci a été noté précédemment, on peut voir que les premières tempêtes de l'hiver 2013-2014, et notamment celle du début du mois de janvier, sont survenues alors que le système dune/haut de plage était considérablement conforté par des apports sédimentaires continus réalisés entre le printemps 2008 et l'hiver 2012-2013 (fig. 14). Ce phénomène de résilience post-tempête du 10 mars 2008 observé sur la plage du Vougot (Suanez et al., 2012b), mais également en baie de Saint-Michel-en-Grève (Suanez et Stéphan, 2011), sur le Sillon de Talbert (Stéphan et al., 2012), en rade de Brest (Stéphan et Laforge, 2013), ou dans l'archipel de Molène (Suanez et al., 2011), a joué dans le faible recul du trait de côte enregistré sur la majeure partie des sites durant l'épisode du début du mois de janvier. Cela montre que la réponse morphosédimentaire des plages à une série d'évènements extrêmes est aussi dépendante des phénomènes de régénération du système littoral agissant entre deux tempêtes.

\section{Conclusion}

29Le suivi topo-morphologique post-tempête réalisé sur l'ensemble des sites répartis le long du littoral sud et nord de la péninsule bretonne montre que la réponse des cordons littoraux 
aux évènements extrêmes de l'hiver 2013-2014 a été particulièrement synchrone. Si des différences existent notamment en matière d'ampleur du recul du trait de côte, elles sont avant tout liées à l'orientation de la houle incidente ; les cordons du sud Bretagne étant plus réactifs aux épisodes d'ouest-sud-ouest, ceux de la côte nord et ouest aux houles d'ouest-nord-ouest. Ces aspects morphodynamiques avaient déjà été observés lors de la tempête du 10 mars 2008. De façon générale, l'ampleur de l'érosion pour ces quatre mois d'hiver (du mois de décembre 2013 au mois de mars 2014), dépasse très largement les reculs épisodiques observés dans le cadre des suivis de plage menés depuis plus de 15 ans. De toute évidence, l'hiver 2013-2014 constitue une rupture majeure dans la dynamique morphosédimentaire à long terme des cordons littoraux bretons. Le recul important de la côte a également relancé les débats autour de la question de la défense du littoral dans beaucoup de communes où les enjeux ont été directement affectés. Pour autant, les observations réalisées après la tempête du 10 mars 2008 ont montré qu'une majeure partie des systèmes littoraux qui avaient été fortement érodés a connu une longue phase de régénération par la suite. Ces phénomènes de résilience assez spectaculaires ont été en grande partie expliqués par la succession d'hivers peu morphogènes que l'on a connu durant quatre ans entre 2008-2009 et 2011-2012. Il serait souhaitable que de telles conditions prévalent de nouveau pour les années à venir car, comme le montre cette étude, la réponse morphosédimentaire des plages à une série d'évènements extrêmes est bien plus dépendante de la morphologie pré-tempête du système littoral que de l'effet cumulé de ces derniers.

\section{Bibliographie}

Des DOI (Digital Object Identifier) sont automatiquement ajoutés aux références par Bilbo, l'outil d'annotation bibliographique d'OpenEdition.

Les utilisateurs des institutions abonnées à l'un des programmes freemium d'OpenEdition peuvent télécharger les références bibliographiques pour lesquelles Bilbo a trouvé un DOI.

Aagaard T., Kroon A., Andersen S., Møller Sørensen R., Quartel S., Vinther N. (2005) Intertidal beach change during storm conditions; Egmond, The Netherlands. Marine Geology 218, 65-80. doi:10.1016/j.margeo.2005.04.001

Aagaard T., Hughes M., Baldock T., Greenwood B., Kroon A., Power H. (2012) Sediment transport processes and morphodynamics on a reflective beach under storm and non-storm conditions. Marine Geology 326-328, 154-165. doi:10.1016/j.margeo.2012.09.004

Almeida L.P., Vousdoukas M.V., Ferreira Ó., Rodrigues B.A., Matias A. (2012) Thresholds for storm impacts on an exposed sandy coastal area in southern Portugal. Geomorphology 143-144, 3-12. doi:10.1016/j.geomorph.2011.04.047

Anthony E.J. (2013) - Storms, shoreface morphodynamics, sand supply, and the accretion and erosion of coastal dune barriers in the southern North Sea. Geomorphology 199, 8-21. doi:10.1016/j.geomorph.2012.06.007

Ardhuin F., Aron R., Dumas F., Bennis A.-C., Sentchev A., Forget P., Wolf J., Girard F., Osuna P., Benoit M. (2012) - Numerical wave modeling in conditions with strong currents: dissipation, refraction and relative wind. Journal of Physical Oceanography 42, 2101-2120. doi:10.1175/JPO-D-11-0220.1 
Armaroli C., Grottoli E., Harley M.D., Ciavola P. (2013) - Beach morphodynamics and types of foredune erosion generated by storms along the Emilia-Romagna coastline, Italy. Geomorphology 199, 22-35. doi:10.1016/j.geomorph.2013.04.034

Balsillie J. (1986) - Beach and coast erosion due to extreme event impact. Shore and Beach 54, 22-37.

Benoit M., Lafon F., Goasguen G. (2006) - Construction et exploitation d'une base de données d'états de mer le long des côtes françaises par simulation numérique sur 23 ans. Actes du colloque des IXèmes Journées Nationales Génie Civil - Génie Côtier, Brest, France, 12-14 septembre 2006. In: L'Her J., Levacher D., Centre Français du Littoral (Eds.), Centre Français du Littoral, Nantes, 21-30. doi:10.5150/jngcgc.2006.003-B

Betts N.L., Orford J.D., White D., Graham C.J. (2004) - Storminess and surges in the south-western approaches of the eastern North Atlantic: the synoptic climatology of recent extreme coastal storms. Marine Geology 210, 227-246. doi:10.1016/j.margeo.2004.05.010

Birkemeier W.A, Nicholls R.J., Lee G.H. (1999) - Storms, storm groups and nearshore morphologic change. Proceedings of the Coastal Sediments'99 Conference, ASCE: 11091122 .

Boak E.H., Turner I.L. (2005) - Shoreline definition and detection: review. Journal of Coastal Research 21, 688-703. doi:10.2112/03-0071.1

Bodéré J.-C., Hallégouët B., Yoni C. (1993) - Erosion et protection des côtes entre Penmarc'h et l'Odet, in Le pays bigouden. A la croisée des chemins, Actes du colloque Pont l'Abbé, 19-21 novembre 1992. Revue Cap Caval, 17, 295-306.

Bosom E., Jiménez J.A., (2010) - Storm-induced coastal hazard assessment at regional scale: application to Catalonia (NW Mediterranean). Advances in Geosciences 26, 83-87. doi:10.5194/adgeo-26-83-2010

Cariolet J.-M., Costa S., Caspar R., Ardhuin F., Magne R., Goasguen G. (2010) Aspects météo-marins de la tempête du 10 mars 2008 en Atlantique et en Manche. Norois, 215, 11-31. doi:10.4000/norois.3242

Caspar R., Costa S., Jacob E. (2007) - Fronts froids et submersions de tempêtes dans le nord-ouest de la France : le cas des inondations par la mer entre l'estuaire de la Seine et de la Somme. La Météorologie, 57, 37-47.

Caspar R., Costa S., Lebreton P., Letortu P. (2010) - Les submersions de tempête de la nuit du 10 au 11 mars 2008 sur la côte d'Albâtre (Haute-Normandie, France) : détermination météo-marine. Norois, 215, 115-133. doi:10.4000/norois.3273

Castelle B., Marieu V., Bujan S., Ferreira S., Parisot J.-P., Capo S., Senechal N., Chouzenoux T. (2014) - Equilibrium shoreline modeling of a high-energy meso-macrotidal multiple-barred beach. Marine Geology 347, 85-94. doi: 10.1016/j.margeo.2013.11.003 
CEREMA (2014) - Caractéristiques générales des houles enregistrées par le houlographe 02911_Les Pierre Noires. Ressource en ligne : http://candhis.cetmef.developpementdurable.gouv.fr/publications/02911/astx_02911_2.pdf

Ciavola P., Armaroli C., Chiggiato J., Valentini A., Deserti M., Perini L., Luciani P. (2007) - Impact of storms along the coastline of Emilia-Romagna: the morphological signature on the Ravenna coastline (Italy). Journal of Coastal Research SI50, 540-544.

Coco G., Sénéchal N., Rejas A., Bryan K.R., Capo S., Parisot J.P., Brown J.A., MacMahan J.H.M. (2014) - Beach response to a sequence of extreme storms. Geomorphology 204, 493-501. doi:10.1016/j.geomorph.2013.08.028

Costa S., Levoy F., Monfort O., Curoy J., De Saint Léger E., Delahaye D. (2008) - Impact of sand content and cross-shore transport on the morphodynamics of macrotidal gravel beaches (Haute-Normandie, English Channel). Zeitschrift für Geomorphologieçi 52, 41-62. doi:10.1127/0372-8854/2008/0052S3-0041

Cox J.C., Pirrello M.A. (2001) - Applying joint probabilities and cumulative effects to estimate storm-induced erosion and shoreline recession. Shore \& Beach 69, 5-7.

Daubord C. (2014) - Caractérisation de 7 évènements de tempête de l'automne-hiver 20132014 à partir des données disponibles au SHOM. Service Hydrographique et Océanographique de la Marine (Brest), N 001/2014 - MIP/HDC/NP Nº 84, 31 p.

Dehouck A., Dupuis H., Sénéchal N. (2009) - Pocket beach hydrodynamics: the example of four macrotidal beaches, Brittany, France. Marine Geology 266, 1-17. doi:10.1016/j.margeo.2009.07.008

Dolan R., Hayden B. (1981) - Storms and shoreline configuration. Journal of Sedimentary Petrology 51, 737-744. doi:10.1306/212F7D92-2B24-11D7-8648000102C1865D

Dubois A., Menier D., Sedrati M. (2014) - Impact morphologique et hydrodynamique d'une période de forte énergie sur le domaine intertidal d'une plage de baie et de trois plages de poche mésotidales en milieu semi-abrité : exemple de la tempête Xynthia en presqu'île de Rhuys (France). Géomorphologie : relief, processus, environnement, 3, 227-242. doi:10.4000/geomophologie.10668

Faye I.B.Nd., Hénaff A., Hallégouët B. (2007) - Evolution récente de la ligne de rivage en baie d'Audierne : de Penhors à la pointe de la Torche. Penn ar Bed, 199/200, 50-61.

Fenster M.S., Dolan R., Morton R.A. (2001) - Coastal storms and shoreline change: signal or noise? Journal of Coastal Research 17, 714-720.

Ferreira Ó. (2005) - Storm groups versus extreme single storms: predicted erosion and management consequences. Journal of Coastal Research SI42, 221-227.

Ferreira Ó. (2006) - The role of storm groups in the erosion of sandy coasts. Earth Surface Processes and Landforms 31, 1058-1060. doi:10.1002/esp.1378 
Fichaut B., Hallégouët B. (1989) - Banneg : une île dans la tempête. Penn ar Bed, 135, 3643.

Fichaut B., Suanez S. (2010) - Dynamiques d'arrachement, de transport et de dépôt de blocs cyclopéens par les tempêtes: le cas de la tempête du 10 mars 2008 sur l'île de Banneg (archipel de Molène, Finistère). Norois, 215, 33-58. doi:10.4000/norois.3224

Fichaut B., Suanez S. (2011) - Carrying, transport and deposition of cliff-top storm deposits during extreme event: Banneg Island, Brittany. Marine Geology 283, 36-55. doi:10.1016/j.margeo.2010.11.003

Forbes D.L., Parkes G.S., Manson G.K., Ketch L.A. (2004) - Storms and shoreline retreat in the southern Gulf of St. Lawrence. Marine Geology 210, 169-204. doi:10.1016/j.margeo.2004.05.009

Garaboeuf M.F. (1990) - Le littoral guissenien. Mémoire de DEA de géographie, Université de Bretagne Occidentale, $73 \mathrm{p}$.

Garnier G. (1993) - Réhabilitation d'un cordon dunaire dégradé. In: Le pays bigouden. A la croisée des chemins. Actes du colloque Pont l'Abbé, 19-21 novembre 1992. Revue Cap Caval, 17, 307-312.

Gervais M., Balouin Y., Belon R. (2012) - Morphological response and coastal dynamics associated with major storm events along the Gulf of Lions Coastline, France. Geomorphology 143-144, 69-80. doi:10.1016/j.geomorph.2011.07.035

Gibeaut J.C., Gutiérrez R., Hepner T.L. (2002) - Threshold conditions for episodic beach erosion along the southeast Texas coast. Gulf Coast Association of Geological Societies Transactions, 52, 323-335.

Haerens P., Bolle A., Trouw K., Houthuys R. (2012) - Definition of storm thresholds for significant morphological change of the sandy beaches along the Belgian coastline. Geomorphology 143-144, 104-117. doi:10.1016/j.geomorph.2011.09.015

Hall A.M., Hansom J.D., Williams D.M., Jarvis J. (2006) - Distribution, geomorphology and lithofacies of cliff-top storm deposits: Examples from the high-energy coasts of Scotland and Ireland. Marine Geology 232, 131-155. doi:10.1016/j.margeo.2006.06.008

Hallégouët B., Bodéré J.-C. (1993) - Un littoral fragilisé, le sud de la baie d'Audierne, in Le pays bigouden. A la croisée des chemins. Actes du colloque Pont l'Abbé, 19-21 novembre 1992. Revue Cap Caval, 17, 263-272.

Hallégouët B., Hénaff A. (1993) - Evolution du littoral septentrional du pays bigouden entre Penhors et Pors Poulhan, In: Le pays bigouden. A la croisée des chemins. Actes du colloque Pont l'Abbé, 19-21 novembre 1992. Revue Cap Caval, 17, 273-280.

Hallégouët B., Hénaff A. (2006) - Evolution récente et gestion des espaces littoraux de l'ouest Cornouaille. Les Actes des rencontres de l'ouest Cornouaille «Quelles pistes de développement pour le territoire ?», Mai-juin 2005, Association Ouest Cornouaille Promotion (AOCP), Pont-L'Abbé, 20-34. 
Holliday N.P., Yelland M.J., Pascal R., Swail V.R., Taylor P.K., Griffiths C.R., Kent E. (2006) - Were extreme waves in the Rockall Trough the largest ever recorded? Geophysical Research Letters 33, L05613. doi:10.1029/2005GL025238

Honeycutt M., Crowell M., Douglas B. (2001) - Shoreline-position forecasting: impact of storms, rate-calculation methodologies, and temporal scales. Journal of Coastal Research 17, 721-730.

Houser C., Hapke C., Hamilton S. (2008) - Controls on coastal dune morphology, shoreline erosion and barrier island response to extreme storms. Geomorphology 100, 223-240. doi:10.1016/j.geomorph.2007.12.007

Jiménez J.A., Sancho-García A., Bosom E., Valdemoro H.I., Guillén J. (2012) - Storminduced damages along the Catalan coast (NW Mediterranean) during the period 1958-2008. Geomorphology 143-144, 24-33. doi:10.1016/j.geomorph.2011.07.034

Lee G., Nicholls R.J., Birkemeier W.A. (1998) - Storm-induced profile variability of the beach-nearshore profile at Duck, North Carolina, U.S.A., 1981-1991. Marine Geology 148, 163-177. doi:10.1016/S0025-3227(98)00010-3

Letortu P., Costa S., Cantat O. (2012) - Les submersions marines en Manche orientale : approche inductive et naturaliste pour la caractérisation des facteurs responsables des inondations par la mer. Climatologie, 9, 31-57.

McCallum E., Norris W.J.T. (1990) - The storms of January and February 1990. The Meteorological Magazine 119, 201-210.

Mendoza E.T., Jiménez J.A. (2006) - Storm-induced beach erosion potential on the Catalonian Coast. Journal of Coastal Research SI48, 81-88.

Morton R.A., Gibeaut J.C., Paine J.G. (1995) - Meso-scale transfer of sand during and after storms: implications for prediction of shoreline movement. Marine Geology 126, 161 179.

Morton R.A. (2002) - Factors controlling storm impacts on coastal barriers and beaches - a preliminary basis for near real-time forecasting. Journal of Coastal Research 18, 486-501.

Orford J.D., Jennings S.C., Pethick J. (2003) - Extreme storm effect on gravel dominated barriers. In: Davis R.A.(Ed.) Coastal Sediments'03. CD-ROM published by World Scientific Publishing Corp. and East Meets West Productions, Corpus Christi, Texas, USA. ISBN 981 238-422-7, $12 \mathrm{p}$.

Orford J.D., Jennings S.C. (2007) - Variation in the organisation of gravel-dominated coastal systems: Evidence from Nova Scotia and Southern England. Coastal Sediments'07, New Orleans, American Society of Civil Engineers, 434-448.

Orford J.D. (2011) - Gravel-Dominated coastal barrier reorganisation variability as a function of coastal susceptibility and barrier resilience. Coastal Sediments 11, 1257-1270. 
Orford J.D., Anthony E.J. (2011) - Extreme events and the morphodynamics of graveldominated coastal barriers: strengthening uncertain ground. Marine Geology 290, 41-45. doi:10.1016/j.margeo.2011.10.005

Perherin C., Kergadallan X., Trmal C. (2013) - Analyse des surcotes extrêmes le long des côtes métropolitaines. Rapport du Centre d'Études Techniques Maritimes Et Fluviales et du le Centre d'Études Techniques de l'Équipement (CETE) Méditerranée (avril 2013), 128 p.

Pian S., Menier D., Sedrati M. (2014) - Analysis of morphodynamic beach states along the South Brittany coast. Géomorphologie : relief, processus, environnement, 3, 261-274. doi:10.4000/geomorphologie.10722

Roche A., Baraer F., Le Cam H., Madec T., Gautier S., Jan G., Goutx D. (2014) - Projet VIMERS : une typologie des tempêtes bretonnes pour prévoir l'impact des tempêtes à venir et mieux s'y préparer. Actes du colloque des XIIIèmes Journées Nationales Génie Côtier Génie Civil, Dunkerque, 2-4 juillet 2014, 925-932. doi:10.5150/jngcgc.2014.101

Roland A., Ardhuin F. (2014) - On the developments of spectral wave models: numerics and parameterizations for the coastal ocean. Ocean Dynamics 64, 833-846. doi:10.1007/s10236-014-0711-z

DOI : $\underline{10.1007 / \mathrm{s} 10236-014-0711-\mathrm{Z}}$

Rangel-Buitrago N., Anfuso G. (2011) - Coastal storm characterization and morphological impacts on sandy coasts. Earth Surface Processes and Landforms 36, 1997-2010. doi:10.1002/esp. 2221

Rascle N., Ardhuin F. (2013) - A global wave parameter database for geophysical applications. part 2: model validation with improved source term parameterization. Ocean Modelling 70, 174-188. doi:10.1016/j.ocemod.2012.12.001

Ruz M.-H., Héquette A., Maspataud A. (2009) - Identifying forcing conditions responsible for foredune erosion on the northern coast of France. Journal of Coastal Research SI56, 356360.

Sénéchal N., Coco G., Bryan K.R., Holman R.A. (2011) - Wave runup during extreme storm conditions. Journal of Geophysical Research 116, C07032. doi:10.1029/2010JC006819

Sénéchal N., Coco G., Castelle B., Marieu V. (2015) - Storm impact on the seasonal shoreline dynamics of a meso- to macrotidal open sandy beach (Biscarrosse, France). Geomorphology 228, 448-461. doi:10.1016/j.geomorph.2014.09.025

Splinter K. D., Turner I.L., Davidson M.A., Barnard P., Castelle B., Oltman-Shay J. (2014) - A generalized equilibrium model for predicting daily to interannual shoreline response. Journal of Geophysical Research: Earth Surface 119, 1936-1958. doi:10.1002/2014JF003106

Stéphan P. (2008) - Les flèches de galets de Bretagne : morphodynamiques passée, présente et prévisible. Thèse de doctorat de Géographie, Université de Bretagne Occidentale, Brest, $558 \mathrm{p}$. 
Stéphan P., Suanez S., Fichaut B. (2010) - Franchissement et recul des cordons de galets par rollover. Impact de la tempête du 10 mars 2008 dans l'évolution récente du Sillon de Talbert (Côtes d'Armor, Bretagne). Norois, 215, 59-75. doi:10.4000/norois. 3252

Stéphan P. (2011a) - Quelques données nouvelles sur la mobilité récente et le bilan sédimentaire des flèches de galets de Bretagne. Géomorphologie: relief, processus, environnement, 2, 205-232. doi:10.4000/geomorphologie.9416

Stéphan P. (2011b) - Les flèches de galets de Bretagne : évolution passée, présente et future. Ed. L'Harmattan, Coll. Milieux naturels et Sociétés, Paris, 263 p.

Stéphan P., Suanez S., Fichaut B. (2012) - Long-term morphodynamic evolution of the Sillon de Talbert gravel barrier (Brittany, France). Shore \& Beach 80, 19-36.

Stéphan P., Laforge M. (2013) - Mise au point sur l'évolution géomorphologique et le devenir des flèches de galets du Loc'h de Landévennec (Bretagne, France). Géomorphologie : relief, processus, environnement, 2, 191-208. doi:10.4000/geomorphologie.10246

Stockdon H.F., Sallenger A.H., Holman R.A., Howd P.A. (2007) - A simple model for the spatially-variable coastal response to hurricanes. Marine Geology 238, 1-20. doi:10.1016/j.margeo.2006.11.004

Stone G.W., Liu B., Pepper D.A., Wang P. (2004) - The importance of extratropical and tropical cyclones on the short-term evolution of barrier islands along the northern Gulf of Mexico, USA. Marine Geology 210, 63-78. doi:10.1016/j.margeo.2004.05.021

Suanez S., Fichaut B., Magne R. (2009) - Cliff-top storm deposits on Banneg Island, Brittany, France: effects of giant waves in the Eastern Atlantic Ocean. Sedimentary Geology 220, 12-28. doi:10.1016/j.sedgeo.2009.06.004

Suanez S., Cariolet J.-M. (2010) - L'action des tempêtes sur l'érosion des dunes : les enseignements de la tempête du 10 mars 2008. Norois, 215, 77-99. doi:10.4000/norois.3212

Suanez S., Stéphan P. (2011) - Effects of Natural and Human Forcing on Mesoscale Shoreline Dynamics of Saint-Michel-en-Grève Bay (Brittany, France). Shore \& Beach 79 (2), 19-38.

Suanez S., Fichaut B., Magne R., Ardhuin F., Corman D., Stéphan P., Cariolet J.-M. (2011) - Changements morphologiques et bilan sédimentaire des formes fuyantes en queue de comète de l'archipel de Molène (Bretagne, France). Géomorphologie : relief, environnement, processus, 2, 187-204. doi:10.4000/geomorphologie.9397

Suanez S., Garcin M., Bulteau T., Rouan M., Lagadec L., David L. (2012a) - Les observatoires du tait de côte en France, EchoGéo, 19, édition électronique. doi:10.4000/echogeo.12942

Suanez S., Cariolet J.-M., Cancouët R., Ardhuin F., Delacourt C. (2012b) - Dune recovery after storm erosion on a high-energy beach: Vougot beach, Brittany (France). Geomorphology 139-140, 16-33. doi:10.1016/j.geomorph.2011.10.014 
Trzpit J.-P. (1977) - Les tempêtes nord-Atlantiques : essai d'analyse géographique $\left(1^{\text {re }}\right.$ partie). Norois, 93, 33-52. doi:10.3406/noroi.1977.3558

Vousdoukas M.I., Almeida L.P.M., Ferreira Ó. (2012) - Beach erosion and recovery during consecutive storms at a steep-sloping, meso-tidal beach. Earth Surface Processes and Landforms 37, 583-593. doi:10.1002/esp.2264

Yates M.L., Guza R.T. O'Reilly W.C. (2009) - Equilibrium shoreline response: Observations and modeling. Journal of Geophysical Research 114, C09014. doi:10.1029/2009JC005359

Zielinski G.A. (2002) - A Classification Scheme for Winter Storms in the Eastern and Central United States with an emphasis on Nor'easters. Bulletin of the American Meteorological Society 83, 37-51. doi:10.1175/1520-0477(2002)083

\section{Annexe}

\section{Abridged English version}

Between December 2013 and March 2014, a cluster of about 12 storm events hit the coast of Brittany with an exceptional frequency. Such frequency and intensity of stormy events had not been recorded since the winter 1989-1990. It was in February that these storm events were the most frequent and particularly virulent. The significant wave heights measured off Finistère reached respectively $12.3 \mathrm{~m}$ and $12.4 \mathrm{~m}$ during both Petra and Ulla storms of February $5^{\text {th }}$ and $14^{\text {th }}$; maximum wave height reached $23.5 \mathrm{~m}$ during Petra storm of February $5^{\text {th }}$ (fig 3). However, analysis of hydrodynamic conditions shows that only three episodes promoted extreme morphogenetic conditions because they were combined with high spring tide level (tab. 4). The first one concerns the period extending from $1^{\text {st }}$ to $5^{\text {th }}$ of January 2014 during which spring tide levels were exceptionally high, especially on January the $3^{\text {rd }}$. During this period, offshore waves were oriented W-SW reaching significant and maximum heights respectively greater than $8 \mathrm{~m}$ and $15 \mathrm{~m}$, in particular between January 3 and 4. Instantaneous storm surges were between $0.30 \mathrm{~m}$ and $0.50 \mathrm{~m}$, but generally higher in southern Brittany (fig. 4 and tab. 3). They were generated by strong winds reaching between 18 and $20 \mathrm{~m} / \mathrm{s}$ speed, and atmospheric pressures of about $996 \mathrm{~Pa}$. Tidal levels were higher than MHWL and/or HAT, especially in southern Brittany (tab. 4). The second episode occurred at the beginning of February, between $31^{\text {st }}$ of January and $4^{\text {th }}$ of February 2014. It was also characterized by the combination of storm event and high spring tide level, especially between $1^{\text {st }}$ and $2^{\text {nd }}$ of February. Offshore wave was oriented W-NW, the significant wave heights reached $7 \mathrm{~m}$ to more than $9 \mathrm{~m}$, and maximum heights over $14 \mathrm{~m}$. Instantaneous storm surge were between $0.20 \mathrm{~m}$ to $0.40 \mathrm{~m}$; the highest surges levels occurred at the end of the period, especially during the Petra storm of February $4^{\text {th }}$ when they reached $0.76 \mathrm{~m}$ (fig. 4 and tab. 3). Storm winds were blowing from west $\left(270^{\circ}\right.$ to $\left.271^{\circ}\right)$ reaching speeds between $16-17$ $\mathrm{m} / \mathrm{s}$, and $25-26 \mathrm{~m} / \mathrm{s}$ on February $4^{\text {th }}$ while atmospheric pressure was $998 \mathrm{~Pa}$ to $993 \mathrm{~Pa}$ (minimum $982 \mathrm{~Pa}$ ). Tide levels were largely above MHWL and/or HAT, with a climax on February $1^{\text {st }}$. The third morphogenetic episode extended from February $28^{\text {th }}$ to March $4^{\text {th }}$, during which Christine storm of $1^{\text {st }}-2^{\text {nd }}$ of March occurred during a spring tide. Offshore waves were oriented $\mathrm{W}-\mathrm{NW}$ and the significant and maximum heights reached respectively over $9 \mathrm{~m}$ and almost $16 \mathrm{~m}$, especially the $3^{\text {rd }}$ of March. Instantaneous storm surges reached 0.5 meters (fig. 4 and tab. 3) while the average speed wind blowing from SW was between 20 
and $22.4 \mathrm{~m} / \mathrm{s}$. Atmospheric pressures were between $982 \mathrm{~Pa}$ and $983 \mathrm{~Pa}$. Again in this case, tidal levels were higher than HAT for all Brittany coast.

Morphological measurements were conducted on more than ten coastal sections located around Brittany peninsula (fig. 2) to evaluate the effects of these storms on shoreline erosion. These surveys were achieved on different types of morphology such as sandy beach/dune systems, gravel barrier spit, shingle beaches, using DGPS. Frequency of measurements was high (twice a month) in order to characterize the impact of major storms (tab. 5). They show that during the first phase (December-January), particularly characterized by the morphogenetic storm event of $1^{\text {st }}$ to $4^{\text {th }}$ January 2014, shoreline erosion has been limited, with the exception of southern Brittany (fig. 6A). This is due to the SW orientation of waves. For all monitoring sites, it has averaged $-2.7 \mathrm{~m}$, the averaged minimum equal to $0.6 \mathrm{~m}$, and the averaged maximum at $-6.20 \mathrm{~m}$. During the second phase from mid-January to mid-February, characterized by $1^{\text {st }}-2^{\text {nd }}$ of February storm corresponding to the most morphogenetic event of the winter, the average of shoreline retreat reached $-4.2 \mathrm{~m}$, the averaged minimum reached approximately $-1.5 \mathrm{~m}$, the averaged maximum $-9.5 \mathrm{~m}$ (fig. 6A). It is essentially the Northern and Western coast of Brittany that experienced largest shoreline retreat due to W-NW storm wave orientation. During the third and last phase, running from mid-February to mid-March, and characterized by the March $2^{\text {nd }}-3^{\text {rd }}$ extreme morphogenetic event, shoreline retreat was very low. It reached $-1 \mathrm{~m}$ on average, for an average minimum of $-0.6 \mathrm{~m}$ and an average minimum of $-1.9 \mathrm{~m}$. Considering the whole winter 2013-14 period, shoreline erosion for all monitoring sites reached $-6.3 \mathrm{~m}$ on average, with a minimum of about $-0.2 \mathrm{~m}$ and a maximum of $-30.1 \mathrm{~m}$ (fig. 6B). Depending on the type of environment, it appears that the dunes have retreated the most, followed by gravel or sandy barriers; the lowest erosion rates concern beaches backed by low cliffs formed by highly consistent materials such as periglacial deposits (head). The morphological response in terms of shoreline retreat of beaches and barriers to the three morphogeneous episodes was different. Storm occurring at the beginning of February during the second phase induced largest erosive rates partly explained by the large morphological sensitivity of beaches and barriers which were weakened by the previous storm event at the beginning of January. Conversely, the storm of March induced very few impacts. These elements show that there is no cumulative storm effect of storm attested on shoreline retreat, but that beyond a certain threshold in the shoreline retreat process, the erosive action of morphogenesis events is no longer significant, regardless of their intensity.

\section{Table des illustrations}

Fig. 1 - Localisation des fenêtres de calcul des paramètres statistiques de houle issues du modèle PREVIMER. Fig. 1 - Location of windows for wave statistic parameters calculation provided by PREVIMER modeling.

Crédits Source : $\underline{\text { http://www.previmer.org Source }: \text { http://www.previmer.org }}$

URL http://geomorphologie.revues.org/docannexe/image/11104/img-1.jpg

Fichier image/jpeg, 772k

Titre

Tab. 1 - Inventaire des tempêtes de l'hiver 2013-2014. Tab. 1 - Inventory of 
winter 2013-14 storms.

Les hauteurs moyennes et maximum des houles sont obtenues à partir de modèles issus du système d'observations et de prédictions PREVIMER

Légende (http://previmer.fr). Wave heights (average and maximum) are obtained from wave modelling produced in a frame of PREVIMER observations and forecasts system (http://previmer.fr).

URL http://geomorphologie.revues.org/docannexe/image/11104/img-2.jpg

Fichier image/jpeg, 3,8M

Titre

Fig. 2 - Carte de localisation des différents sites d'étude. Fig. 2 - Location map of study sites.

URL http://geomorphologie.revues.org/docannexe/image/11104/img-3.jpg

Fichier image/jpeg, 1,4M

Fig. 3 - Caractéristiques de la houle basée sur les enregistrements réalisés au large du Finistère par la bouée Datawell des Pierres Noires, et marées observées pour la côte nord, ouest et sud de la Bretagne, entre les mois de décembre 2013

Titre et mars 2014. Fig. 3 - Wave characteristics based on waverider measurements achieved off western Brittany peninsula (Pierres Noires buoy), and tide measurements achieved for the North, West and South Brittany coast, between December 2013 and April 2014.

A : localisation de la bouée Datawell des Pierres Noires et des marégraphes de Roscoff, du Conquet et de Concarneau ; B : hauteurs et directions de la houle ; marée observée au Conquet $(C)$, à Roscoff $(D)$, à Concarneau (E), comparée au niveau des pleines mers de vive-eau et au niveau des plus hautes mers

Légende astronomiques. A: Location map of Pierres Noires wave buoy, and Roscoff, Le Conquet, and Concarneau tide gauge stations; B: Wave height and direction; Observed tide measurement at Le Conquet (C), Roscoff (D), Concarneau (E) compared with Mean High Water Spring and High Astronomic Tide levels for each location.

URL http://geomorphologie.revues.org/docannexe/image/11104/img-4.jpg

Fichier image/jpeg, 2,7M

Fig. 4 - Analyse des surcotes enregistrées par les marégraphes de Roscoff pour la côte nord de la Bretagne (A), du Conquet pour la pointe occidentale (B), et de Concarneau pour la côte méridionale (C), comparées aux niveaux de surcote maximum pour des périodes de retour de 5, 20, 50 et 100 ans. Fig. 4 - Surge analysis recorded by tide gauge station of Roscoff for North Brittany coast (A), of Conquet for Western Brittany coast (B), and Concarneau for South Brittany coast (C), compared to maximum surge levels for 5-yr, 20-yr, 50-yr, and 100-yr return periods. 
URL $\quad \underline{\text { http://geomorphologie.revues.org/docannexe/image/11104/img-5.jpg }}$

Fichier image/jpeg, 1,2M

Tab. 2 - Estimation des surcotes extrêmes calculées selon la loi d'ajustement GPD (loi de Distribution Généralisée de Pareto) à partir des enregistrements

Titre marégraphiques du Conquet, de Roscoff et de Concarneau. Tab. 2 - Estimation of extreme surges calculated using the GPD fit (generalized Pareto distribution) from the tide gauge records of Conquet, Roscoff and Concarneau.

Crédits Source : Perherin et al., 2013. Source: Perherin et al., 2013.

URL http://geomorphologie.revues.org/docannexe/image/11104/img-6.jpg

Fichier image/jpeg, 524k

Tab. 3 - Niveaux d'eau maximum et surcotes instantanées enregistrés par les marégraphes de Roscoff, du Conquet, et de Concarneau, pour les épisodes

Titre tempétueux survenus durant l'hiver 2013-2014. Tab. 3 - Maximum water level and instantaneous surge calculated using records of Conquet, Concarneau and Roscoff tide gauge stations during winter 2013-14 storm events.

Les lignes en grisé illustrent les épisodes durant lesquels la surcote maximum

Légende instantanée a coïncidé avec une pleine mer. Shaded lines show the episodes where instantaneous maximum surge coincided with high tide level.

URL http://geomorphologie.revues.org/docannexe/image/11104/img-7.jpg

Fichier image/jpeg, 3,8M

Tab. 4 - Caractéristiques hydrodynamiques et météorologiques des trois Titre épisodes tempétueux les plus morphogènes de l'hiver 2013-2014. Tab. 4 Hydrodynamic and meteorological characteristics of the three most morphogeneous storm events of the winter 2013-2014.

URL http://geomorphologie.revues.org/docannexe/image/11104/img-8.jpg

Fichier image/jpeg, 1,5M

Fig. 5 - Approche méthodologique suivie pour la définition d'une limite bioTitre morphologique du trait de côte. Fig. 5 - Methodological approaches to the definition of shoreline indicator based on bio-morphological limits.

A : exemple d'un cordon dunaire dont tout le versant externe a reculé ; B : exemple d'un cordon dunaire dont seule une partie du versant externe a reculé ; $C$ : exemple d'un cordon dunaire partiellement submergé sans qu'il n'y ait de

Légende recul de l'édifice. A: Example of a dune which experienced complete retreat of the seaward slope; B: Example of a dune from which only part of seaward slope has retreated;C: Example of a dune partially submerged without erosion phenomena. 
URL $\quad \underline{\text { http://geomorphologie.revues.org/docannexe/image/11104/img-9.jpg }}$

Fichier image/jpeg, 2,0M

Tab. 5 - Inventaire des levés topomorphologiques effectués durant l'hiver 2013-

Titre 2014. Tab. 5 - Inventory of topomorphological measurements achieved during the 2013-2014 winter.

URL http://geomorphologie.revues.org/docannexe/image/11104/img-10.jpg

Fichier image/jpeg, 1,1M

Fig. 6 - Recul du trait de côte $(A)$ généré par les trois épisodes morphogènes du début du mois de janvier, Février et mars 2014, (B) à l'échelle de l'hiver 20132014. Fig. 6 - Shoreline retreat $(A)$ induced by the three morphogenetic events of early January, February and March 2014, (B) for the whole winter 2013-2014.

URL http://geomorphologie.revues.org/docannexe/image/11104/img-11.jpg

Fichier image/jpeg, 2,7M

Fig. 7 - Illustration des trois phases de recul du trait de côte pour le cordon

Titre littoral sableux de la plage du Vougot (Guissény - nord Finistère). Fig. 7 - Three phases of shoreline retreat of Vougot beach sandy coast (Guissény - North Finistère).

URL http://geomorphologie.revues.org/docannexe/image/11104/img-12.jpg

Fichier image/jpeg, 2,1M

Fig. 8 - Illustration des trois phases de recul du trait de côte de la baie de Saint-

Titre Michel-en-Grève (Côtes d'Armor). Fig. 8 - Three phases of shoreline retreat for Saint-Michel-en-Grève Bay (Côtes d'Armor).

URL http://geomorphologie.revues.org/docannexe/image/11104/img-13.jpg

Fichier image/jpeg, 3,0M

Fig. 9 - Illustration des trois phases de recul du trait de côte pour le cordon

Titre littoral sableux Treffiagat-Léchiagat (sud Finistère). Fig. 9 - Three phases of shoreline retreat of Treffiagat-Léchiagat beach (South Finistère).

URL http://geomorphologie.revues.org/docannexe/image/11104/img-14.jpg

Fichier image/jpeg, 1,8M

Fig. 10 - Illustration des trois phases de recul du trait de côte par rollover de la

Titre flèche de galets du Sillon de Talbert (Côtes d'Armor). Fig. 10 - Three phases of shoreline retreat by rollover of gravel spit of Sillon de Talbert (Côtes d'Armor). 
URL http://geomorphologie.revues.org/docannexe/image/11104/img-15.jpg

Fichier image/jpeg, 1,4M

Fig. 11 - Illustration des trois phases de recul du trait de côte du cordon de

Titre galets de la plage de Porz sur l'île de Triélen - archipel de Molène (ouest Finistère). Fig. 11 - Three phases of shoreline retreat by rollover shingle beach of Porz on Triélen Island (Western Finistère).

URL http://geomorphologie.revues.org/docannexe/image/11104/img-16.jpg

Fichier image/jpeg, 548k

Fig. 12 - Erosion du trait de côte et destruction des ouvrages de défense du littoral de la plage Boutrouilles à Kerlouan - nord Finistère $(A)$, et de la plage de

Titre Léhan à Treffiagat-Léchiagat - sud Finistère (B). Fig. 12 - Shoreline erosion and destruction of coastal protection structures on Boutrouilles beach at Kerlouan North Finistère (A), and Léhan beach at Treffiagat-Léchiagat - South Finistère (B).

URL http://geomorphologie.revues.org/docannexe/image/11104/img-17.jpg

Fichier image/jpeg, 3,1M

Fig. 13 - Erosion du trait de côte et destruction des aménagements littoraux de

Titre la plage de Porsmilin - ouest Finistère. Fig. 13 - Shoreline erosion and destruction of coastal development on Porsmilin beach - Western Finistère.

A : variation du trait de côte et changement de profils de plage ; $B$ : situation avant les tempêtes le $09 / 03 / 2004 ; C$ : et le $29 / 10 / 2012$; D : situation après

Légende tempête le 02/02/2014.A: Shoreline and beach profile changes; B: situation before storm events 09/03/2004; C: and 29/10/2012; D: situation after storm events 02/02/2014.

URL http://geomorphologie.revues.org/docannexe/image/11104/img-18.jpg

Fichier image/jpeg, 2,7M

Fig. 14 - Evolution du bilan sédimentaire du cordon dunaire de la plage du

Titre Vougot (Guissény) dans sa partie orientale entre les mois de juin 2004 et septembre 2014. Fig. 14 - Sediment budget evolution of the dune of the eastern part of Vougot beach (Guissény) between June 2004 and October 2014.

URL http://geomorphologie.revues.org/docannexe/image/11104/img-19.jpg

Fichier image/jpeg, 1,5M 